This document is downloaded from DR-NTU (https://dr.ntu.edu.sg) Nanyang Technological University, Singapore.
A biomechanical model of human upper limb for objective stroke rehabilitation assessment

Ang, Wei Sin

2018

http://hdl.handle.net/10356/73470

https://doi.org/10.32657/10356/73470 


\section{A BIOMECHANICAL MODEL OF HUMAN UPPER LIMB FOR OBJECTIVE STROKE REHABILITATION ASSESSMENT}

\section{ANG WEI SIN}

School of Mechanical \& Aerospace Engineering

A thesis submitted to the Nanyang Technological University in partial fulfilment of the requirements for the degree of Doctor of Philosophy 


\section{Acknowledgement}

I would like to express my sincere gratitude to my advisors Professor Chen IMing and Professor Hartmut Geyer for their invaluable advice and support throughout all these years during my graduate studies. Professor Chen has been instrumental in the selection of the research topic during my first years in Nanyang Technological University (NTU), providing insights and guidance that broadened my horizon in the area of research. When I first arrived at Carnegie Mellon University (CMU), I was at the crucial phase of my research and at the same time handling the coursework and settling in with my family. Professor Geyer knew about my situation, helped by providing a conducive research environment, and spent time going through with me the difficulties I faced in my research. When I was preparing for this thesis and a submission of a journal paper, he would help to vet through the drafts and provide valuable feedback to make my writings better. For all these and more, I am grateful to have both professors as my thesis advisors.

In the course of my graduate studies, I am fortunate to have met many people that had in one way or another made my journey a more fruitful and enjoyable one. I would like to thank Professor Jessica Zhang and Professor Koushil Sreenath for agreeing to be in my thesis committee despite their busy schedules, and providing insightful feedback during my proposal. During my time in NTU, I have to privilege to befriend some fellow students and researchers in the Robotic Research Centre. In particular, I would like to express my appreciation to Yuan Qilong, Lum Guozhan, Albert Causo, and Shee Cheng Yap. I had benefitted very much from the discussions we had and the helps I received from them. When I was in CMU, the fellow members of Prof Geyer's group made me feel at home and welcomed, making the tough transition into a smooth process. I would like to thank all of them, especially Seungmoon Song, William Martin, Albert Wu and Nitish Thatte. Last but not least, I would also like to express my gratitude to all of whom had helped me during my stay in Pittsburgh, especially Wee Liat 
Ong, Goh Chun Fan, Rodney and Suzanne, Tay Junyun, Zhuang Jiang, and Ye Zhou. 


\begin{abstract}
In stroke rehabilitation, the assessments of the severity of stroke that are based on objective and robust measurements are the key to improve the efficacy of the rehabilitation efforts. It is essential, therefore, to complement the existing tools, where the assessments are partly relied on therapists' subjective judgements, with a tool that can quantify important indicators of stroke recovery. One such indicator is the level of spasticity. The reliability of the current methods of measuring the severity of spasticity can be significantly improved by incorporating a feasible way to measure muscle forces and activations during stroke assessment. However, most of the present methods of estimating muscle forces require input parameters that are difficult to obtain in a clinical setting. A musculoskeletal arm model has been developed to bridge the gap between the domains of muscle forces estimation and stroke rehabilitation assessment.
\end{abstract}

The project is divided into three stages. In the first stage, a biomechanical arm model that computes the joint torques with kinematic data from sensors is developed. The model has three features that eliminate the need for parameters that are difficult to obtain thus making it a feasible tool in clinical settings. The first is the use of a hybrid method that combines the data from sensors and a shoulder rhythm model to compute the orientation of the shoulder complex. The second is a method to compute the elbow joint angles without the need to compute the ambiguous carrying angle. The third is a method of estimating the inertial properties using published data, scaled by parameters that can be easily measured.

The musculoskeletal properties of the human arm are added to the model in the second stage. The muscle model consists of 22 muscles that span from the thorax via the shoulder and the upper arm to the forearm. The muscle path is defined using Obstacle Set method where the anatomical structures are modelled using regular-shaped rigid bodies. Dynamics of the muscle is computed based on the 
Hill's type muscle model that consists of an active contractile element, a passive parallel element and a series element. Due to the difficulties in defining the moment arms, an optimization routine is designed to compute the optimal moment arms for each muscle for a subject. The muscle-sharing problem is solved using optimization which minimises the square of sum of muscle stresses. The muscle activation predicted by the model is compared to EMG signal for validation.

In the final stage of this project, the model is used in the application of spasticity assessment. The tonic stretch reflex threshold (TSRT) which is an indicator for the severity of spasticity is computed using the model. Fifteen patient subjects participated in the experiments where they were assessed by two qualified therapists using Modified Ashworth Scale (MAS), and their motions and EMG signals were captured at the same time. Using the arm model, the TSRT of each patient was measured and ranked. The estimated muscle activation profiles have a high correlation (0.707) to the EMG signal profiles. The null hypothesis that the rankings of the severity using the model and the MAS assessment have no correlation has been tested, and was rejected convincingly ( $\mathrm{p} \approx 0.0003)$. These findings suggest that the model has the potential to complement the existing practices by providing an alternative evaluation method. 


\section{Contents}

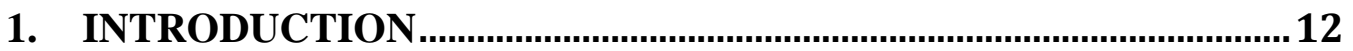

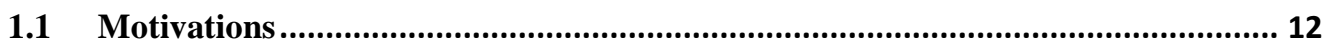

1.1.1 The need for robotics and automation technologies in stroke rehabilitation ..... 12

1.1.2 Assessment in rehabilitation of the upper limbs of stroke patients and its

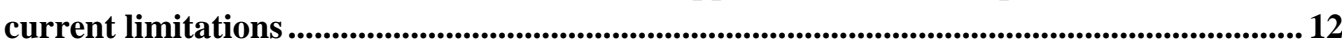

1.1.3 Estimation of Muscle Activations and Its Limitations on Using in Stroke

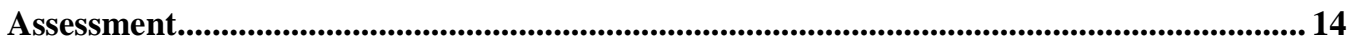

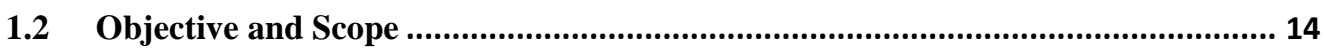

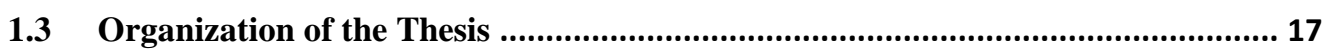

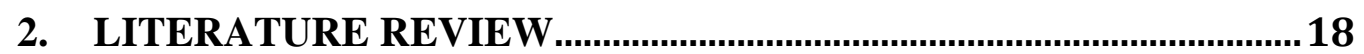

2.1 Assessment of Motor Recovery after Stroke....................................................... 18

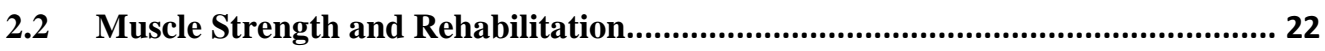

2.3 Measurement of Muscle Strength .................................................................... 23

2.3.1 Manual Muscle Test (MMT) ...................................................................................... 23

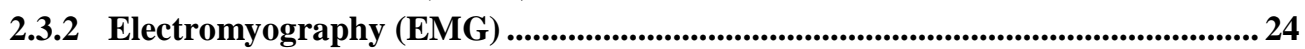

2.3.3 Model-based Estimation.......................................................................................... 25

\section{A BIOMECHANICAL MODEL FOR THE HUMAN UPPER LIMB} 29

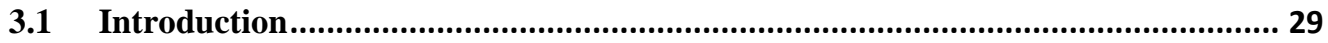

3.1.1 Joint Kinematics ............................................................................................ 29

3.1.2 Sensors Setup .................................................................................................... 30

3.2 The Denavit-Hartenberg (D-H) Representation of the Upper Limb Model......... 33

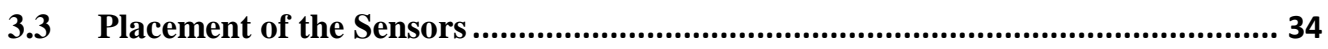

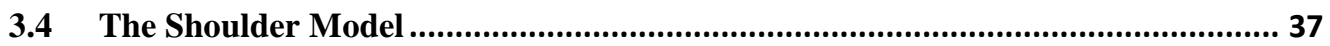

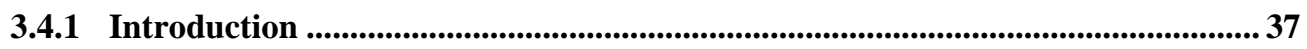

3.4.2 Shoulder Rhythm Model................................................................................. 38

3.4.3 Measurement of the orientations of scapula and humerus using IMUs.............. 40

3.4.4 Fusion of data from regression models and sensor measurements for scapular

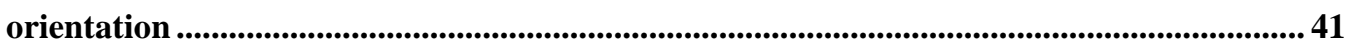

3.4.5 Validating Shoulder Model ............................................................................. 42

The Elbow Model ......................................................................................... 48

3.5.1 Estimation of the rotation axes of the forearm..............................................48

3.5.2 Decomposition of joint angles at the elbow..........................................................5 50

3.5.3 Validating the elbow model............................................................................... 51

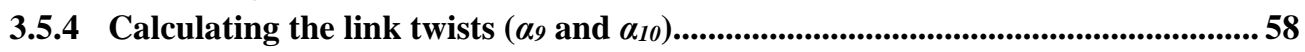

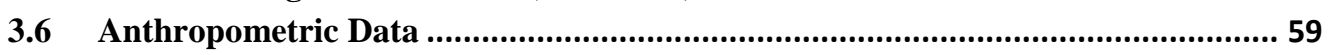

3.6.1 Measurable Parameters ...................................................................................5 59

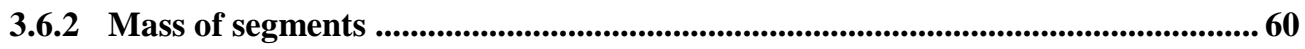

3.6.3 Center of mass (COM) of segments..............................................................60 
3.6.4 Moments of inertial (MOI) of segments............................................................... 61

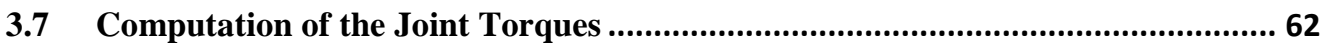

3.7.1 Linear Kalman filter..........................................................................................6 62

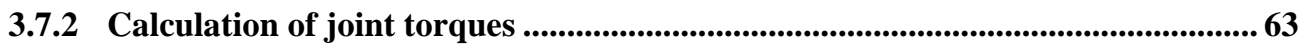

3.7.3 Validation and experimental results .........................................................64 64

\section{A MUSCULOSKELETAL MODEL FOR THE HUMAN UPPER}

LIMB

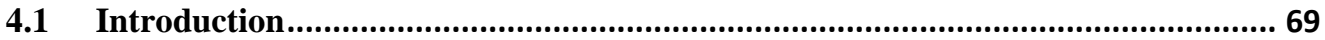

4.2 Defining origins and insertions of the muscles................................................69

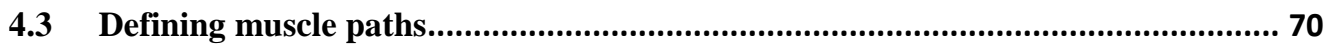

4.4 Muscle contraction dynamics model ............................................................... 71

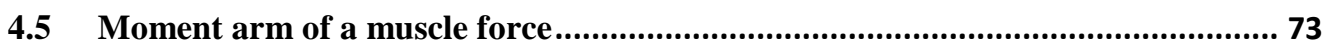

4.6 Muscle force sharing problem ........................................................................ 75

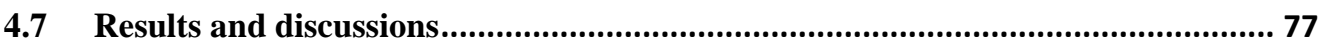

5. APPLICATION: ASSESSMENT OF SPASTICITY ……………….....82

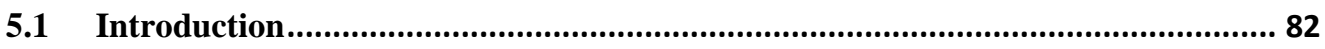

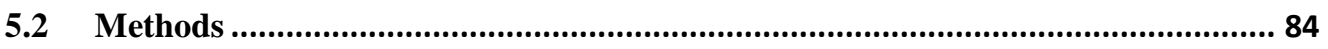

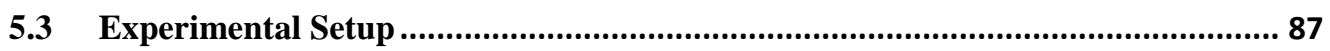

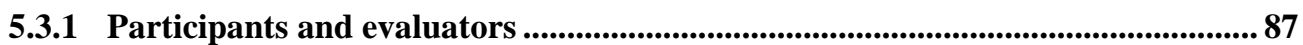

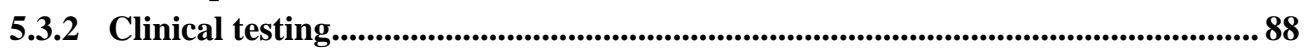

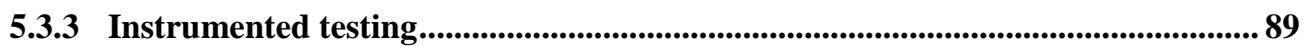

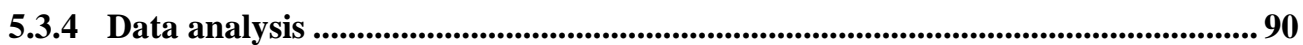

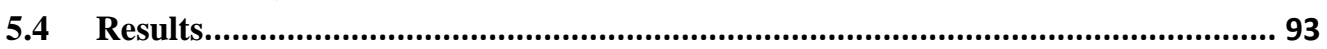

5.4.1 Validation of model using EMG .....................................................................93 93

5.4.2 Comparison between model predictions and clinical results ............................... 94

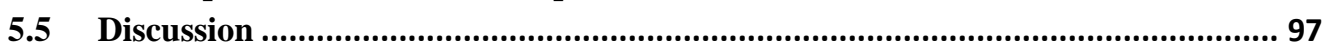

5.5.1 Validation of the Model using EMG and Its Implications ...............................97

5.5.2 The Correlation between Model Prediction and the Clinical Assessments and Its

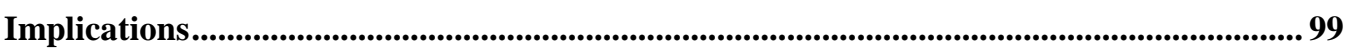

5.6 Future Works for Spasticity Assessments .................................................. 99

6 CONCLUSION AND FUTURE WORKS........................................ 101

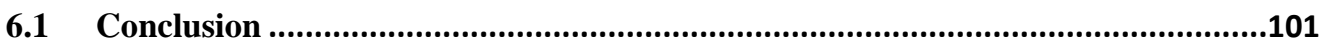

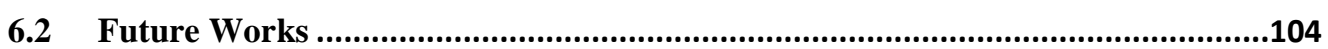

APPENDIX A: LIST OF MUSCLES AND THEIR OBSTACLE-SET

PARAMETERS ............................................................................................. 106

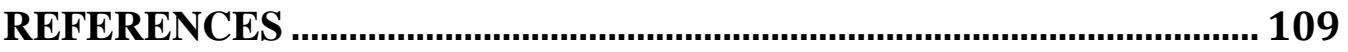




\section{Figures}

Figure 1: The three stages of the project. Stage 1 - Biomechanical arm model takes in measured data and computes joint torques using inverse dynamics. Stage 2 Muscle model incorporates joint torques and estimates muscle force/activation via optimization. Stage 3 - Practical applications. ................................................... 16

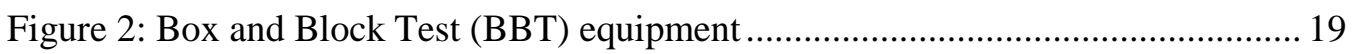

Figure 3: Action research Arm Test (ARAT) equipment ......................................... 19

Figure 4: Manual Muscle Testing in progress............................................................. 23

Figure 5: A schematic of measuring EMG using skin-attached electrodes. .................. 25

Figure 6: The inverse and forward dynamics models.................................................. 26

Figure 7: The general form of a Hill-type muscle model, and the force-length and force-

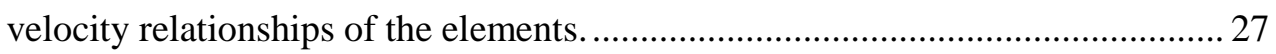

Figure 8: (a) A electromagnetic tracking system (Flock of Birds ${ }^{\mathrm{TM}}$, Ascension Technology Corp.); (b) An optoelectronic tracking system with markers on subject (Vicon $^{\mathrm{TM}}$, Oxford Metrics Limited); (c) A electrogoniometer adhered to the forearm; and (d) Inertial measurement units attached to the body of a subject. 31

Figure 9: The illustration of the arm model that consists of 4 segments (clavicle, scapula, humerus and forearm), 4 joints (SC, AC, GH and Elbow), and a base (Thorax). The rotation axes, $Z_{n}$, in the $\mathrm{D}-\mathrm{H}$ representation are also shown. 33

Figure 10: The coordinate systems and placement of the IMUs, the global frame, and the local frames. Note that the local frames of the clavicle and scapula are not shown.

Figure 11: Anatomy of the shoulder. It consists of three bones: clavicle, scapula and humerus, and the thorax as the base. The three synovial joints are sternoclavicular (SC), acromioclavicular (AC) and glenohumeral (GH). The scapula glides along the ribcage forming the scapulothoracic articulation (ST). 37

Figure 12: The relationship between $\alpha$ (in Eq. 3.9) and measured humeral elevation angle, $\Delta \boldsymbol{\theta H m}$. 42

Figure 13: Clavicular $\left(\theta_{C X}, \theta_{C Y}\right.$, and $\left.\theta_{C Z}\right)$ and scapular $\left(\theta_{S X}, \theta_{S Y}\right.$, and $\left.\theta_{S Z}\right)$ orientations versus humeral elevation angle $\left(\theta_{H}\right)$ of one subject raising right arm in (a) the sagittal plane, and (b) the frontal plane; and (c) versus time-step for shrugging. Solid lines are the fused angles; dotted lines are the regression model data; and dot-dashed lines are the measured data from the IMUs. 46

Figure 14: Clavicular $\left(\theta_{C X}, \theta_{C Y}\right.$, and $\left.\theta_{C Z}\right)$ and scapular $\left(\theta_{S X}, \theta_{S Y}\right.$, and $\left.\theta_{S Z}\right)$ orientations versus movement cycle in (a) drinking, and (b) combing motions of all four subjects. The black lines are the corresponding angular profiles in [82] (for the drinking motion the cycle ends at $50 \%$ cycle of our experiment). No result of clavicular angles were presented in [82] 47

Figure 15: The calculated $S_{F E}$ and $S_{P S}$ of one subject shown in the frontal plane. The dashed lines are the axes calculated between two consecutive positions. The solid 
lines are the optimal axes. The axes are dimensionless as only the directions of the axes are plotted.

Figure 16: The plot of the trajectories of $q_{F E}$ (left column) and $q_{P S}$ (right column) versus the percent of movement cycle of one subject: (a) \& (b) flexion; (c) \& (d) supination/pronation ; (e) \& (f) arm raised in the sagittal plane; and (g) \& (h) drinking water. .55

Figure 17: Comparison of (a) $q_{F E}$ and (b) $q_{P S}$ during the drinking movement among

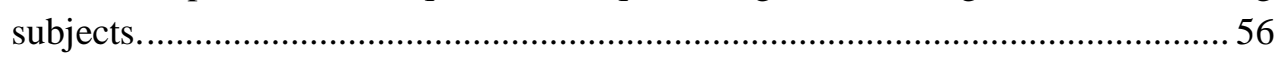

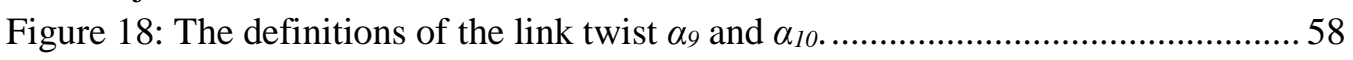

Figure 19: Profiles of torque at dominant DOFs during arm raising in: (a) Sagittal plane (DOFs: SC posterior rotation and AC posterior tilting); (b) Frontal plane (DOFs: SC elevation and AC lateral rotation). 66

Figure 20: The regular-shaped rigid bodies used as obstacles in obstacle-set method: single sphere, single cylinder, double cylinder and sphere-capped cylinder. ....... 71

Figure 21: Three-element Hill type muscle model consists of a contractile element (CE) and two non-linear spring elements, parallel element (PE) and series element (SE). $l_{C E}$ is the length of CE and $l_{S E}$ is the length of SE, both add up to the length of the muscle element, $l_{m} . F_{m}$ is the muscle force.

Figure 22: The placement of EMG sensors on the deltoid, the biceps brachii, the triceps brachii, and the brachioradialis. 77

Figure 23: Muscle activation level (\%MVC) predicted by the model and EMG signals of four arm muscles during weight-lifting motion. The blue line is the average muscle force of six trials and the green lines are the standard deviation of the trials. The dotted line is the corresponding EMG signal. .78

Figure 24: Muscle activation level (\%MVC) predicted by the model and EMG signals of four arm muscles during drinking motion. The blue line is the average muscle force of six trials and the green lines are the standard deviation of the trials. The dotted line is the corresponding EMG signal. 79

Figure 25: DSRTs are measured at different angular velocities and TSRT is estimated at the zero velocity via extrapolation of the linear regression. The maximum flexed position, $\theta^{-}$, and the maximum extended position, $\theta^{+}$, define the biomechanical range of the joint. For healthy subjects, the TSRT is beyond the biomechanical range. 84

Figure 26: Elbow extension assessment for spasticity. The therapist supports the patient's elbow with one hand while extending the forearm with the other hand. 85

Figure 27: Placement of the three APDM Opal ${ }^{\mathrm{TM}}$ wireless IMUs on the upper limb... 90

Figure 28: Muscle activations of six groups of muscles predicted by the model at a medium speed of about $75^{\circ} / \mathrm{sec}$. The muscle activation is computed in \%MVC and plotted against the \% completion of the extension motions. The muscles selected cover the forearm (Brachioradialis), upper arm (Biceps and Deltoid), shoulder anterior (Subscapularis) and shoulder posterior (Rhomboid Major and Minor)... 92

Figure 29: The linear regression of the DSRT_B of Participant 3. TSRT_B is the $\mathrm{x}$ intercept of the regression line. .93 
Figure 30: Model prediction of muscle activation and linear envelope of EMG signal of biceps brachii of Participant 3 at a speed of about 162\%sec. The EMG envelope was normalized such that the 2SDs above the mean baseline coincides with the 1\%MVC of the model prediction. The cross-correlation between the two profiles for this particular test is 0.84 . .94 


\section{Tables}

Table 1: Stroke Rehabilitation Assessment Tools ...................................................... 18

Table 2: The Modified Ashworth Scale ................................................................. 21

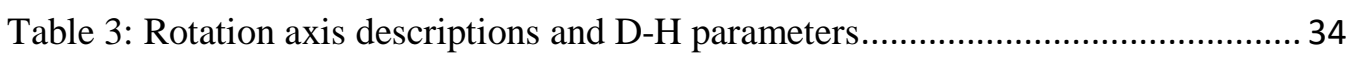

Table 4: Positions of IMUs and orientations of the frames.......................................... 36

Table 5: Regression models of sternoclavicular (SC) joint based on results in [106].

$\theta_{C X}, \theta_{C Y}$, and $\theta_{C Z}$ are the orientation of the clavicle with respect to the thorax in the $x_{C}, y_{C}$, and $z_{C}$ axes respectively. $\theta_{H}$ is the humeral elevation angle measured in the three different elevation planes $\left(90^{\circ}, 40^{\circ}, 0^{\circ}\right.$ with respect to the frontal plane). For $\theta_{C X}$, the variations are insensitive to the elevation plane. All angles are in degree.

Table 6: Regression models of scapulothoracic (ST) joint based on results in [106]. $\theta_{S X}$, $\theta_{S Y}$, and $\theta_{S Z}$ are the orientation of the scapula with respect to the thorax in the $x_{S}$, $y_{S}$, and $z_{S}$ axes respectively. $\theta_{H}$ is the humeral elevation angle measured in the three different elevation planes $\left(90^{\circ}, 40^{\circ}, 0^{\circ}\right.$ with respect to the frontal plane). For $\theta_{S X}$ and $\theta_{S z}$, the variations are insensitive to the elevation plane. There no significant change in $\theta_{S Y}$ when the arm is abducted in the frontal plane. All angles

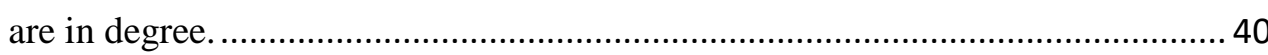

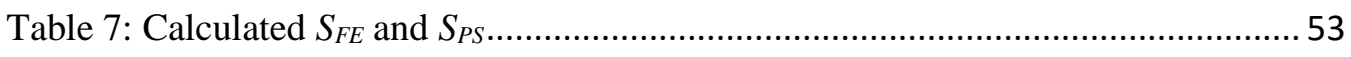

Table 8: Orientational deviation between the calculated and measured poses, $\Delta R$...... 57

Table 9: The values of $N_{Q}$ and $N^{i}{ }_{R}$ (approximated from data in [108]) ........................63

Table 10: Maximum Joint Torques during Arm Anteflexion (Sagittal Plane)..............67 67

Table 11: Maximum Joint Torques during Arm Abduction (Frontal Plane) .................67

Table 12: Cross-correlation coefficients, $r_{A E}$, between estimated muscle activation level and EMG signal level of each muscle for the weight-lifting and drinking motions.

Table 13: Demographic information of the participants

Table 14: The model prediction for TSRTs and the clinical assessment results in Modified Ashworth Scale (MAS). TSRT Biceps uses the activation of biceps brachii while TRST Others uses the earliest activation of any of the six groups of muscles (brachioradalis, biceps brachii, deltoid, subscapularis, rhomboid major and rhomboid minor). The model predictions are ranked based on the TSRT (in \%Motion). The MAS is ranked first by the score $(0,1,1+, 2,3,4)$ for elbow extension tests. The ties are settled using MAS scores for shoulder, wrist and thumb, in that sequence.

Table 15: The results of the null hypothesis, $H_{0}$, that the model prediction of the severity of spasticity via TSRT has no correlation to the clinical assessments via MAS. The hypothesis testing makes use of Spearman's rank correlation test via $t$ test, with $n=15$ and significance level, $\alpha=0.05$. The null hypothesis is rejected if $p$-value $<\alpha$. 


\section{Introduction}

\subsection{Motivations}

\subsubsection{The need for robotics and automation technologies in stroke rehabilitation}

Stroke patients often suffer from both sensory and motor difficulties in one or more limbs. Besides the initial huge medical costs incurred in treating the patients, the survivors of stroke require intensive care and the road to recovery through rehabilitation is a long and expensive one [1]. This is partly because the current common practice of rehabilitation of stroke patients is a combination of both physical and occupational therapy [2], which requires at least a one-to-one attention of a trained therapist to a patient [3]. To alleviate the financial and manpower burden on the healthcare sector, hospitals and rehabilitation centres are exploring the possibilities of automating some of the rehabilitation procedures, taking advantage of emerging robotics and automation technologies [4-6].

\subsubsection{Assessment in rehabilitation of the upper limbs of stroke patients and its current limitations}

The assessments of the severity of stroke in patients during rehabilitation that are based on objective and robust measurements are the key to justify evidence-based practice and serve as a tool for communication among researchers and practitioners [7]. It is also important that the tool used for objective assessment is feasible in a clinical setting so that the practitioners can use it without the need to invest in expensive equipment and challenging training. The assessments are needed in three situations: (a) to plan for the best initial treatment methods, (b) to quantify the efficacy of the treatment sessions so that they could be fine-tuned progressively, and (c) to assess the recovery status of the patients after rehabilitation [8]. There are many tools for stroke assessment [9], each was 
developed to cater for different needs identified by clinicians and therapists. Some of the tools are very simple and are only meant to be used for getting a quick and general overview of the conditions of the patient. One example is the Box and Block Test (BBT). The assessment results of these tools cannot be used for in-depth understanding of the patient's impairment. On the other hand, more complex assessment tools, for example the Fugl-Meyer Assessment (FMA) and the Modified Ashworth Scale (MAS), may suffer from the problem of subjectivity. That is, the result of the assessment partly relies on subjective judgments, which is dependent on the experience of the therapist administrating the test. The inability of these tools to fully address the complexity of the motor deficits also reveals their limitations as a mean to understand the issues pertaining to the stroke recovery process. A more objective and comprehensive assessment method that provides quantitative results would be a valuable tool to complement the existing practices. In particular, researchers have attempted to develop tools to objectively quantify one of the most important indicators of stroke recovery: the level of spasticity.

Most of the current tools developed by researchers to quantify the level of spasticity make use of kinematic data of the arm measured by sensors. Since spasticity is defined as a motor disorder characterized by a velocity-dependent exaggeration of tonic stretch reflexes [10], researchers attempted to quantify the level of spasticity by measuring the joint velocities and electromyography (EMG) signals of muscles to deduce the patient's tonic stretch reflex threshold (TSRT) [11-14]. Due to selectivity issues inherent to using non-invasive EMG sensors, only the activities of the largest superficial arm muscles like biceps brachii or triceps brachii can be measured $[12,13]$. However, it was shown by Mullick et al. in [14] that TSRT measurement is sensitive to the muscles that are been measured and the placement of the sensors. To eliminate these reliability issues, it is required to develop a better method that can determine the activation of more muscles, including smaller and deeper muscles. Without the dynamic information of the muscles during the motions, it is also very difficult to understand the 
underlying mechanisms of the motor deficits and thus hindering the selection of appropriate motor rehabilitation strategies for individual patients [15]. The key in resolving the problems in quantifying spasticity lies in deriving a feasible way to measure muscle forces and activations during stroke assessment.

\subsubsection{Estimation of Muscle Activations and Its Limitations on Using in Stroke Assessment}

Researchers have developed many methods to estimate muscle forces and activations, but there are still gaps to bridge before they can be used directly in the assessment of stroke rehabilitation. First, direct measurement of muscle force is only possible in a few specific muscles $[16,17]$. So instead of measuring the muscle force directly, many researchers develop musculoskeletal models of human extremities to estimate the muscle forces [18]. However, the arm models in these studies are developed to specifically address certain research problems. In some cases, they are over-simplified and do not represent the anatomy accurately to be used for estimation of the muscle strength [19, 20], and in others, the models require input parameters that are difficult to obtain in a rehabilitation setting [21-23]. Some models are developed using commercial software where costs and the trainings needed for the clinicians to use the model are also a hindrance to make these methods useful in assessing stroke rehabilitation [18].

\subsection{Objective and Scope}

The main objective of the project is to bridge the gap between the current modelbased methods for estimating muscle force, which are complex and mainly restricted to the research domain, and a tool that can be used clinically in stroke assessment, which is of low cost, requires simple parameters, and accessible to a broader group of users. This is achieved by the development of a musculoskeletal model of the human upper limb that: (1) requires input parameters that can be obtained in a clinical setting; and (2) has accuracy comparable to the existing models. By providing a mean to estimate the muscle forces/activations using only 
simple protocols, we can objectively quantify the level of spasticity of patients in a clinical setting. We hypothesize that there are significant correlations between the level of severity of stroke predicted using the model and by the current practices.

The complete project consists of three stages: (1) developing a biomechanical arm model; (2) incorporating musculoskeletal features to the model; and (3) applying the model to quantify spasticity (See Figure 1). In the first stage of the project, we developed a biomechanical arm model that takes in the orientation information provided by four sensors attached to the subject's body, and computes the joint torques using inverse dynamics. The four sensors measure the movements of the thorax, scapula, humerus and forearm respectively. In the second stage, we use the joint torques together with a musculoskeletal model for the arm and estimate the muscle forces and activations via optimization techniques. The final stage is to use the complete model to assess the severity of stroke by measuring TSRT to quantify the level of spasticity. Fifteen patient subjects participated in the experiment where we compare the severity of spasticity predicted using the model with the assessments in Modified Ashworth Scale (MAS) done by experienced therapists.

The main contributions of this project are summarized as follows: (a) Developed a biomechanical model of the human upper limb that computes the shoulder orientation accurately using a hybrid method and the elbow orientation without invoking orthogonality and carry angle assumptions; (b) Developed a musculoskeletal model (that incorporates the aforementioned biomechanical model) that is designed to be useful clinically for the assessment of stroke rehabilitation; (c) Applied the model to assess the severity of spasticity of stroke patients and achieved a good correlation with the assessment by professional therapists. 
The research works were published in the following papers:

1. Wei Sin Ang, I-Ming Chen, Qilong Yuan, “Ambulatory measurement of elbow kinematics using inertial measurement units”, IEEE/ASME International Conference on Advanced Intelligent Mechatronics, (AIM), 2013

2. Wei Sin Ang, I-Ming Chen, Qilong Yuan, “Ambulatory measurement of shoulder kinematics using inertial measurement units and shoulder rhythm model”, 3rd IFToMM International Symposium on Robotics and Mechatronics, (ISRM), 2013

3. Wei Sin Ang, Hartmut Geyer, I-Ming Chen, “Objective Assessment of Spasticity with a Method based on a Human Upper Limb Model”, IEEE Transactions on Neural Systems \& Rehabilitation Engineering, 2017 (Accepted).

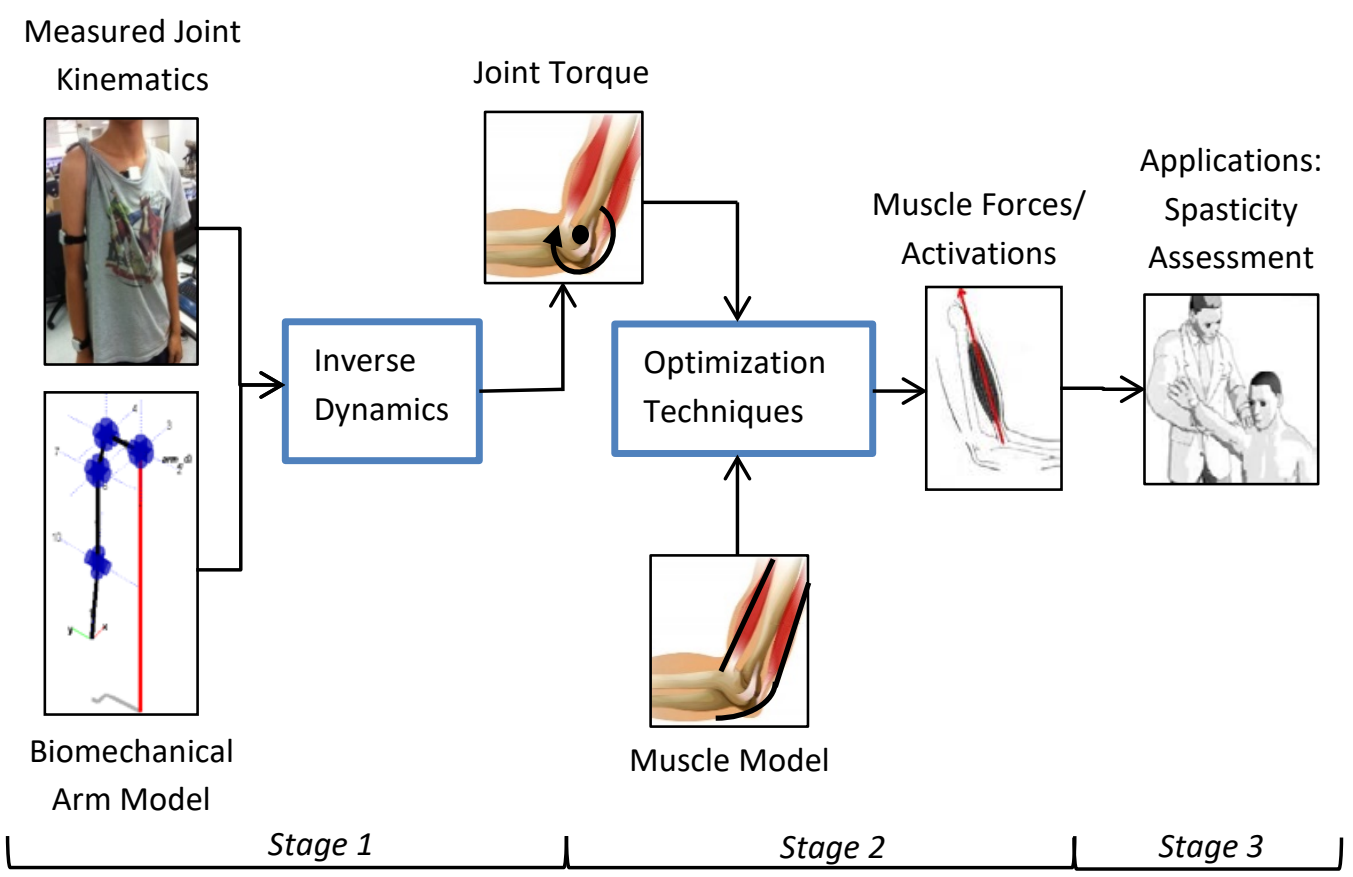

Figure 1: The three stages of the project. Stage 1 - Biomechanical arm model takes in measured data and computes joint torques using inverse dynamics. Stage $2-$ Muscle model incorporates joint torques and estimates muscle force/activation via optimization. Stage 3 - Practical applications. 


\subsection{Organization of the Thesis}

This report presents the modelling of the arm in Stage 1 and 2, and discusses the methods and results of the application in Stage 3. Chapter 2 provides a thorough literature review on the current status of stroke rehabilitation assessment and muscular activation measurement. The development of a biomechanical model of the human upper limb (Stage 1) is presented in Chapter 3. Chapter 4 shows the incorporation of muscular features into the arm model (Stage 2), and estimation of the muscular activations using the joint torques computed from Stage 1. Chapter 5 describes the methods used in applying the arm model to assess spasticity through the measurement of tonic stretch reflex threshold (TSRT). It also presents the experimental results and discusses the implications. Chapter 6 concludes the report and discusses the future works. 


\section{Literature Review}

\subsection{Assessment of Motor Recovery after Stroke}

The survivors of stroke often suffer from upper limb paresis that can have severe impact on disability and health [24]. Assessment of the severity of the impairment before, during and after therapeutic treatment is necessary to identify the best rehabilitation program for individuals and whether the treatment has achieved its purpose [25]. As there are large variations in the characteristics and scope of upper limb recovery among individuals [26, 27], there are over 60 different assessment tools that are been used by clinicians and therapists [9]. The tools are grouped under specific domains that the assessors are interested in. See Table 1 for some the domains that are relevant to motor recovery and the more commonly used tools.

Table 1: Stroke Rehabilitation Assessment Tools

\begin{tabular}{|c|c|c|}
\hline Domains & Tools & Notes \\
\hline \multirow{2}{*}{$\begin{array}{c}\text { Activities of Daily Living } \\
\text { (ADL) }\end{array}$} & $\begin{array}{l}\text { Assessment of Motor and } \\
\text { Process Skills (AMPS) [28] }\end{array}$ & $\begin{array}{l}\text { Effects of motor and process } \\
\text { skills on ADL. }\end{array}$ \\
\hline & Barthel Index (BI) [29] & $\begin{array}{l}\text { The extent to which a patient } \\
\text { can function independently. }\end{array}$ \\
\hline \multirow[t]{2}{*}{ Motor Function } & $\begin{array}{l}\text { Fugl-Meyer Assessment } \\
\text { (FMA) [30] }\end{array}$ & $\begin{array}{l}\text { Designed to assess motor } \\
\text { functioning, balance, } \\
\text { sensation and joint } \\
\text { functioning. }\end{array}$ \\
\hline & Motor Assessment Scale [31] & $\begin{array}{c}\text { Performance-based scale } \\
\text { designed to assess everyday } \\
\text { motor function. }\end{array}$ \\
\hline \multirow{2}{*}{ Upper Extremity Activity } & $\begin{array}{c}\text { Action Research Arm Test } \\
\text { (ARAT) [32] }\end{array}$ & $\begin{array}{l}\text { Assess specific change in } \\
\text { upper limb functions. }\end{array}$ \\
\hline & $\begin{array}{c}\text { Box and Block Test (BBT) } \\
\text { [33] }\end{array}$ & $\begin{array}{l}\text { Measures unilateral gross } \\
\text { manual dexterity. }\end{array}$ \\
\hline Spasticity & $\begin{array}{l}\text { Modified Ashworth Scale } \\
\text { (MAS) [34] }\end{array}$ & $\begin{array}{c}\text { A rating scale to measure } \\
\text { tonus abnormality. }\end{array}$ \\
\hline
\end{tabular}

The BBT is a simple exercise that aims at measuring the subject's gross manual dexterity of one arm. It is composed of a wooden box divided in two compartments by a partition and 150 blocks. See Figure 2. The subject is asked 
to move, one by one, the maximum number of blocks from one compartment of a box to another of equal size, within 60 seconds, and the score equals the number of blocks moved. Because of its simplicity, it is routinely used by clinicians to have a quick and general assessment on the recovery progress of the patients. Although it was found to have good reliability and validity [35], the lack of complexity of BBT means that it cannot provide in-depth understanding of conditions of the subject, which can be seen in the floor-effect when compared to other tests like FMA [32].

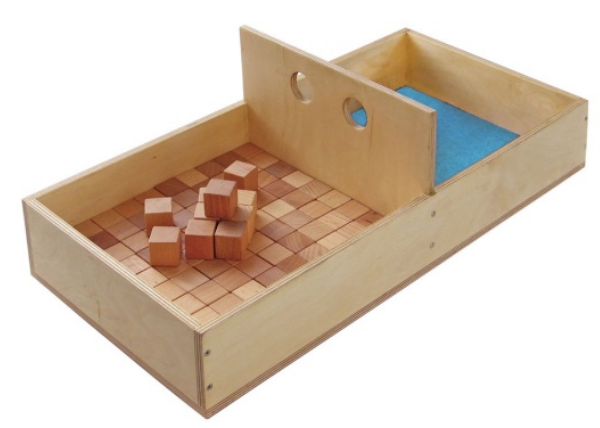

Figure 2: Box and Block Test (BBT) equipment

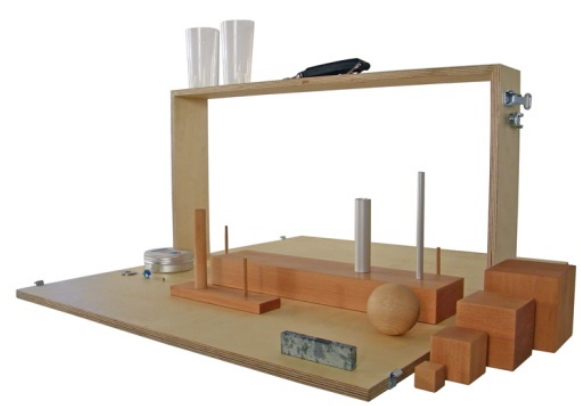

Figure 3: Action research Arm Test (ARAT) equipment

In contrast to BBT's simple equipment, ARAT consists of 19 items (see Figure 3) grouped into four subscales: grasp, grip, pinch, and gross movement, and all items are ordered according to ascending difficulty. The subject is to attempt the most difficult tasks first and will only proceed to the easier ones if he/she cannot complete the task with full score. Although ARAT seems to be more complex 
and detailed than BBT, and studies have shown that it has good reliability and validity [36], it also susceptible to both floor and ceiling-effect [37].

The FMA is one of the most popular assessment tools in the field of stroke rehabilitation and many consider it to be the most comprehensive quantitative measures of motor impairment [38]. The FM scale consists of five domains: motor function, sensory function, balance, joint range of motion, and joint pain. Each item within the domains is assessed using a 3-point scale (0, 1 or 2$)$, with a total score of 226 points. FMA was designed with the intention to fill in the voids in the neuromuscular capacity in most existing scales at the time of its inception [30]. The main merits of FMA are given to the motor function domain of the scale [38, 39], where there are 33 items on the upper extremity and 17 items on the lower extremity. Its reliability and validity have been shown to be very good $[39,40]$, and it has been used as a benchmark for other test scales [32].

Spasticity is a condition that results from injury to the central nervous system. Basically, there is a velocity dependent increase in muscle tone (resistance to stretch) due to excessive contraction of the muscles [41]. The MAS is the most commonly used clinical measures of spasticity [42]. During the test, the limb of the patient is passively moved by the therapist from extreme flexed position to extreme extended position, or vice versa, in one second. The resistance to the passive movement is then graded by the therapist using a set of rules, shown in Table 2. Its reliability is found to range from excellent [43] to poor [44]. When it is validated against biomechanical measurement (using a force transducer and a electrogoniometer), the correlation is found to be poor [41].

Dynamic stretch reflex threshold (DSRT) is defined as the joint angle at which the involuntary resistance to the motion set in at a certain velocity. Higher stretch velocities result in smaller DSRT. If we use linear regression to approximate the DSRTs of various velocities and extrapolate to zero velocity, we will get the tonic stretch reflex threshold (TSRT), which is used as an indicator of the severity of the spasticity [12]. To improve the reliability and objectivity of using the MAS, researchers have attempted to objectively quantify the patient's TSRT by 
simultaneously measuring the joint velocity and the muscle activation via electromyography (EMG) signals [11-14]. Due to the selectivity and representativeness issues related to non-invasive, surface EMG (sEMG) measurement [45], only the activities of large and superficial muscles like biceps brachii or triceps brachii can be reliably recorded [12, 13]. It has been shown, however, that the deduced TSRT is sensitive to the selection of muscles and the placement sites of the sEMG electrodes [14].

Table 2: The Modified Ashworth Scale

\begin{tabular}{|c|c|}
\hline Grade & Modified Ashworth Scale [34] \\
\hline 0 & No increase in muscle tone \\
\hline 1 & $\begin{array}{l}\text { Slight increase in muscle tone, manifested by a catch and release or by } \\
\text { minimal } \\
\text { resistance at the end of the range of motion when the affected part(s) is } \\
\text { moved in } \\
\text { flexion or extension }\end{array}$ \\
\hline $1+$ & $\begin{array}{l}\text { Slight increase in muscle tone, manifested by a catch, followed by minimal } \\
\text { resistance throughout the remainder (less than half) of the range of motion } \\
\text { (ROM) }\end{array}$ \\
\hline 2 & $\begin{array}{l}\text { More marked increase in muscle tone through most of the ROM, but } \\
\text { affected part(s) easily moved }\end{array}$ \\
\hline 3 & Considerable increase in muscle tone, passive movement difficult \\
\hline 4 & Affected part(s) rigid in flexion or extension \\
\hline
\end{tabular}

Despite having some progresses in justifying the usages of these common assessment tools, and therapists and clinicians all over the world are using them routinely in helping the patients, recovery from stroke is still notoriously difficult to quantify [46, 47]. Some of the reasons for this are the difficulty in differentiating recovery by rehabilitation from patient's spontaneous recovery, the variability in the types and severities of the stroke, and the experience and subjectivity of the therapists that administrate the tests [48]. Measurements that are robust and objective are the key to justify evidence-based practice and serve as a tool for communication among researchers and practitioners [7]. The World Health Organization International Classification on Functioning, Disability and Health (WHO ICF) [49] classifies upper limb assessments into three areas: (a) Body Functions and Structures, (b) Activities, and (c) Participation. The most commonly used assessment tools that include BBT, ARAT and FMA, make use 
of clinician-observed and patient self-report methods, and fall mainly into the “Activities" framework [7]. They are not adequate and objective in capturing the dynamic process of recovery and providing indications on the efficacy of the specific treatments $[38,50]$.

\subsection{Muscle Strength and Rehabilitation}

There is no one single parameter that can encompass the complexity and variability of motor recovery after stroke. Muscle strength, however, is a good candidate as the quantity to assess the functional capabilities and general quality of life of stroke patients [51, 52]. Motor deficits are probably the most easily identifiable impairment in stroke patients, and the partial loss in muscle strength is the most noticeable form [53]. Therefore, assessing muscle function is important in many different areas, like determining the existence of an impairment or disability, types and levels of treatment to be administrated, and tracking effectiveness of a treatment [51].

The fact that the motions of the limbs are generated by actions of the muscles means that when the ability to fully activate certain muscles is challenged, as in the case of stroke patients, the acceleration of the corresponding body segments is also compromised [52]. The relevance of muscle strength to functional activities depends on the level of difficulty in performing the activities. Easy tasks like eating, dressing oneself, or combing do not require large strength but more on dexterity of the arm and hand. When an individual has certain difficulties in carry out the motions in a natural trajectory, that is, to perform the task in the same way before the injury, motor compensation will inevitably be employed by the person [54]. Motor compensation typically falls into three areas: derivation of a new function, performing of a function in a new way, and complete a task using a different technique [55]. The quantification of the muscle strength will provide insights to the motor compensation and the comparison of the muscle 
usage between a patient and that of healthy subjects will be very helpful in assessing the recovery status.

\subsection{Measurement of Muscle Strength}

\subsubsection{Manual Muscle Test (MMT)}

The most common method of assessing muscle strength is the manual muscle test (MMT) [56]. (See Figure 4) It is a procedure that evaluates the strength of a particular muscle or muscle group in which the subject voluntarily uses the muscle against some imposed loads or gravity. The body part of the subject under testing is positioned and isolated so that its movement is constrained in the designated direction. Evaluation is then done based on palpation or observation of the contraction of the muscle and the range of motion, and the assignment of scores is purely dependent on the clinical judgment of the therapist [51]. That MMT is one of the most widely used methods to measure muscle strength does not eliminate the fact that its reliability, validity and accuracy are questionable [57]. It is also not useful in ambulatory measurements of daily functions of the limbs and other body parts.

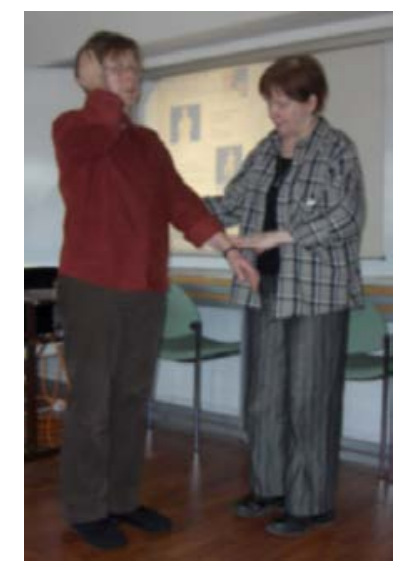

Figure 4: Manual Muscle Testing in progress 


\subsubsection{Electromyography (EMG)}

During muscle contractions, depolarization of the muscle fiber membrane occurs, causing two traveling action potentials that start at the neuromuscular junction halfway the fiber and propagate in opposite directions along the fiber. The membrane depolarization causes a time-varying electric current field that emits an electrical signal known as the electromyogram (EMG). EMG can be measured invasively by means of needle or wire electrodes, or non-invasively at the skin using skin-attached electrodes [45] (see Figure 5). The invasive measurement of EMG has been done for many years by researchers [58]. It can capture electric potentials in a point of the volume conductor close to the active muscle fibers, thus the diffusion effect of the soft tissues is limited. This means that it can measure motor units activation and thus contraction of muscles accurately. However, its usefulness is heavily restricted by the fact that needle-insertion is not feasible in many applications [59]. The non-invasive method, termed as surface EMG (sEMG), on the other hand, suffers the diffusion effects as the skin and other soft tissues sandwiched between the electrodes and the muscles [60, 61]. Recently, there has been a lot of interest in refining the instrumentations and algorithms of sEMG, in the hope of improving its accuracy and precision, so that it can reliably measure muscle force and be used as a clinical tool in the diagnosis of neuromuscular diseases [59, 62, 63]. There are still many hurdles to cross before this can be realized [64], however. These challenges include normalization of EMG amplitudes to maximum muscle force, difficulty in tracking dynamic contraction of muscles [45], and representativeness of EMG due to the heterogeneity of muscle [59]. Currently, EMG signals are only used as a supporting tool that qualitatively validate other muscle force measuring methods [65]. 


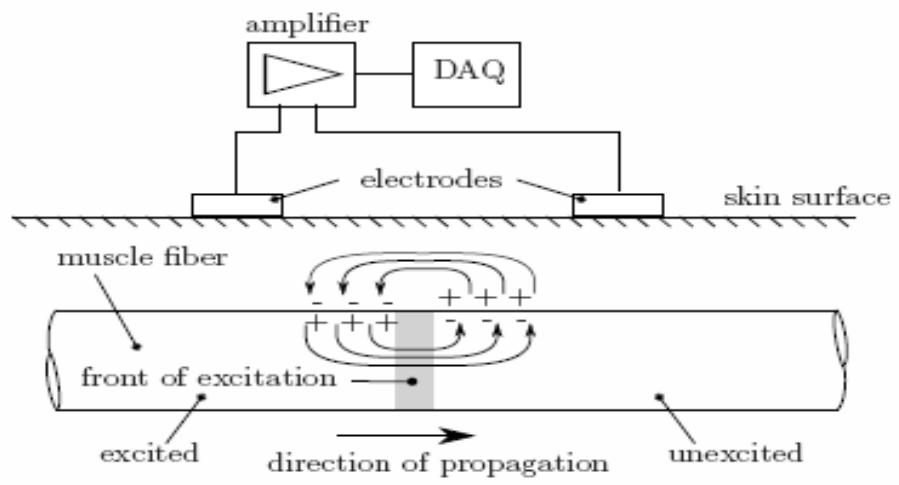

Figure 5: A schematic of measuring EMG using skin-attached electrodes.

\subsubsection{Model-based Estimation}

Direct measurement of muscle force in vivo, when done invasively, can only be used on specific tendons such as Achilles [16], or flexor tendon of fingers [17]. Non-invasive methods, like MMT and EMG measurement described above, either focus on the overall strength or are not precise enough to provide insight into the functions of individual muscle or muscle groups.

Instead of measuring the muscle force directly, many researchers are developing mathematical models of muscle functions [21, 66]. Using quantities that can be relatively easier to measure, like kinematics of the body parts, as inputs to these models, one can calculate the joint torques and then deduce the muscle strengths that are needed to produce the motions [65].

The equations of motion in a musculoskeletal model with a set of $n$ joint angles $q$ can be concisely expressed as [22]:

$$
M(q) \ddot{q}+C(q, \dot{q})+G(q)+T+E=0
$$

where $M(q)$ is the system mass matrix $(n \times n), C(q, \dot{q})$ is the centrifugal and coriolis loading $(n \times 1), G(q)$ is the gravitational loading $(n \times 1), T$ is the muscular joint torque $(n \times 1)$, and $E$ represents external forces. If we know the mass properties and the time history of joint kinematics and reaction forces, we can calculate the joint torques, $T$, by using Eq. ( 2.1 ) and work recursively from 
distal to proximal segments of the body part of interest [67]. This is call inverse dynamics analysis. (See Figure 6.)

If the joint torques are known, usually from experimental data or prior knowledge of the movement, we can rearrange Eq. ( 2.1 ) as

$$
\ddot{q}=M(q)^{-1}[C(q, \dot{q})+G(q)+T+E]
$$

and $\ddot{q}$ can be integrated twice to obtain the joint angles [65]. This approach is called the forward dynamics analysis. (See Figure 6.)

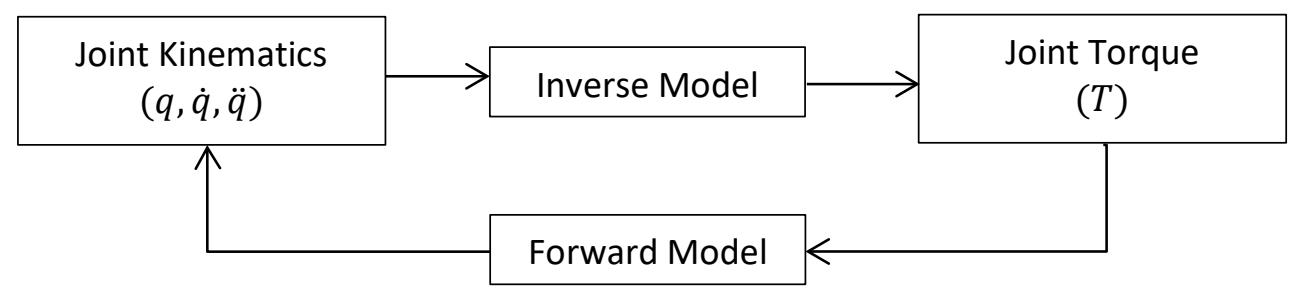

Figure 6: The inverse and forward dynamics models.

The knowledge of the origins and insertions of the muscles of interest is very important as it defines the moment arms, which when multiplied to the corresponding muscle forces give the torques at the joints [68]. Moment arms are defined as the distance between the muscle's line of action and the joint's axis of rotation [69]. The exact locations of the origins and insertions of muscles are usually found using magnetic resonance imaging (MRI) or measurements from cadaveric samples [70]. The former is impractical in a clinical or rehabilitation setting. The latter provides a database from which a subject's actual locations of the sites can be approximated by scaling from the anthropometric information, like height, weight and gender.

The model of muscle can range from very complex, with up to 50 parameters to describe the motion of a single joint [71], to very simple, like being considered as a simple torque generator [72]. The key to the modeling is to make it as complex as needed in the purpose of the studies, such that the model reveals the behavior of interest but does not include parameters that cannot be identified or controlled. Generally, a Hill-type input-output model is a good balance, as it 
considers the force-velocity, force-length and activation dynamics at a whole muscle level [73]. The model consists of an active contractile element (CE), simulating the active muscular action, a passive elastic element (PE), simulating the rigidity of the muscle fiber, and a serial passive element (SE) representing the attached tendons. Figure 7 (taken from [65]) shows the most general form of the model which includes the pennation angle, $\alpha$. The patterns of the force-length and force-velocity relationship are also shown. The muscle force, $F^{m}$ is defined as

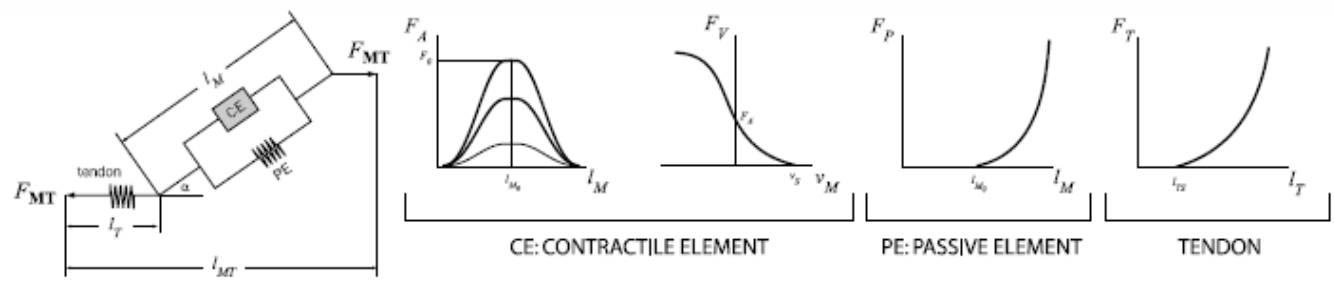

Figure 7: The general form of a Hill-type muscle model, and the force-length and force-velocity relationships of the elements.

$$
F^{m}=F_{C E}^{m}(L(t), v(t), a(t))+F_{P E}^{m}(L(t))
$$

where $F_{C E}^{m}$ and $F_{P E}^{m}$ are the forces from the CE and PE respectively, and they are functions of the muscle length, $L(t)$, the rate of change of the length, $v(t)$, and the muscle activation, $a(t)$.

Musculoskeletal systems are redundant systems, that is, there are many more muscles spanning the joints than the number of joints. Optimization techniques are usually employed to handle the redundancy issues [22]. In the popular inverse dynamics-based static optimization approach [76], the joint torques are first calculated using Eq. (2.1), and the muscular load-sharing problem is solved by minimizing an objective function, $J$, subject to the constraints of equality of the products of muscular forces and moment arms to the joint torques. The objective function, $J$, depends on the nature of the studies and the information available. The most common forms are square of muscle stresses and maximum muscle stress, and their merits and drawbacks are discussed in [65]. 
Another approach is called the forward dynamics assisted data tracking. In this method, an initial set of muscle activation data is fed into the forward dynamics model, and by comparing to the experimental data, the muscle activation information is iteratively updated. The objective function to minimize is the error between the calculated and the measured kinematics of the joints [77]. This approach is less sensitive to the measurement errors of the kinematic inputs when compared to the inverse dynamics model approach. But because it requires the $a$ priori knowledge of muscle activation, which can only be obtained from periodic movement patterns, it is currently only feasible for lower extremity studies [78]. Other alternative approaches include using EMG data incorporated into the calculations, as in the EMG-driven forward dynamics model [79, 80], and combination of EMG data and inverse dynamics model [81]. However, these methods suffer the same problems associated with the ambiguity and inaccuracy inherent to the EMG data, and the procedures and instrumentations are too demanding to be practical in most clinical settings.

In the context of measuring muscle strength to assess rehabilitation of stroke patients, using inverse dynamics model approach seems to be the most feasible, for two reasons: first, it is best suited for upper limb measurements as it does not require a priori information of the muscle activation, which is difficult, if possible, to obtain for the arm as no standard movement pattern is available [82]; secondly, the kinematic data of the arm can be measured relatively easier than the parameters of the muscles, using motion capturing devices like inertial measurement unit (IMU) [83]. But in order for the approach to work, a biomechanical model for the arm that has the appropriate complexity has to be developed first. 


\section{A Biomechanical Model for the Human Upper Limb 3.1 Introduction}

Compared to the researches on the motions of the lower limbs, there are relatively fewer studies in the literature on the upper limb motion analyses [84]. This is because: (a) there is no common or repetitive activity of the arm that can be viewed as a standard motion, unlike walking or running for the legs; and (b) the range of motion of the arm is much greater, and to model it realistically, one has to include the shoulder complex, which has proven to be very difficult to model [85]. Despite these difficulties, many quantitative motion analysis techniques for the upper extremity have been developed over the past twenty years [86]. Each of these studies focuses on addressing different problems, but most of them adopted the same approach of having a biomechanical model of the arm at the central of the investigation, and using measurable quantities captured by sensors to carry out calculations of non-measureable quantities based on the model.

There no standard protocol for arm motion analysis currently recognized by all researchers. However, some of the issues that studies on the arm motions have to address are universal [86].

\subsubsection{Joint Kinematics}

Joint kinematics is the relative attitude of two adjacent bony segments. In the context of the upper limb, it refers to clavicle relative to thorax, scapula relative to clavicle, humerus relative to scapula, ulna or radius relative to the humerus. When developing the model, one has to firstly decide on the degree of freedom (DOF) of each joint, since this will determine the overall complexity of the model and thus the information it can provide. Take the shoulder complex as an example, the DOF designated can range from five [87], six [88], to nine [89], each study considering its own constraints and assumptions made.

The coordinate systems of the joints must be defined with respect to some known references. For the results from different studies to be comparable, anatomical 
frames should be used whenever possible. Anatomical frames are coordinate systems associated with known bony landmarks on the body, usually located by palpation. In [90], the International Society of Biomechanics (ISB) comprehensively proposed a framework on defining anatomical frames for the upper limb motions. However, palpation of the less obvious bony landmarks requires skills of experienced clinicians, or special equipment [91], which may not be readily available in many situations. Another type of frame, called the functional frame, uses functional axes of rotation as the basis for definitions of coordinate systems. One example is using the flexion/extension axis of the elbow to define the forearm coordinate system (see Section 3.5).

The definitions of the joint angles are also a concern when different studies are compared. The ISB recommendations in [90] define these angles with respect to anatomical landmarks. Whenever possible, the joint angles in the model should follow closely to these recommendations, where the Euler angles decompositions are clearly defined.

\subsubsection{Sensors Setup}

The method used to track the motions of the arm is an important aspect in the development of a model, and it has to be decided at the very beginning. This is because the tracking method will determine (a) the type of motions that can be measured, whether it is static, quasi-static, or dynamic; (b) the space and setting of the venue of the experiment; (c) the positions of the markers or wearable sensors on the arm, and whether they can be easily fitted onto the subjects; and (d) the costs. Some common sensors used by researchers are (1) electromagnetic sensors [92, 93] (Figure 8a), (2) optoelectronic systems with passive or active markers [94-96] (Figure 8b), (3) electrogoniometers [97] (Figure 8c), (4) inertial measurement units [98] (Figure 8d), and (5) magnetic resonance imaging (MRI) [99]. Researchers also design and build custom-made measuring tools for specific purposes, for example in [100], a potentiometer-based tool is used in tracking the scapular motions. 
Electromagnetic systems determine the positions of receivers attached to the arm relative to a transmitter. The advantage of these systems is that they do not require line-of-sight, and therefore the position and motions of the subject are relatively less restricted. The major drawback of electromagnetic systems is that it is very sensitive to metals, and such interference should be carefully eliminated during experiments.

Optoelectronic systems do not suffer from sensitivity to metals, but it requires more elaborated setups and the space required is considerably larger. At least three markers are needed for each segment for its rigid body motion to be accurately calculated. More markers or cameras are needed if the movements obstruct the line-of sight of some of the markers.

(a)

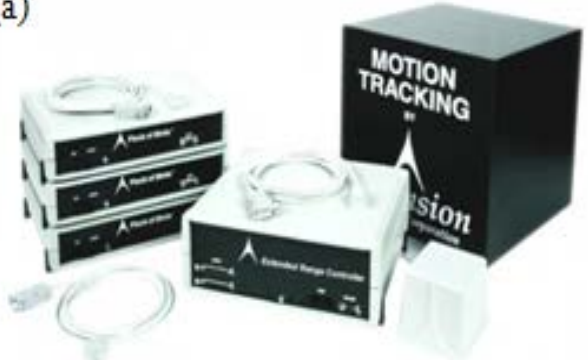

(c)

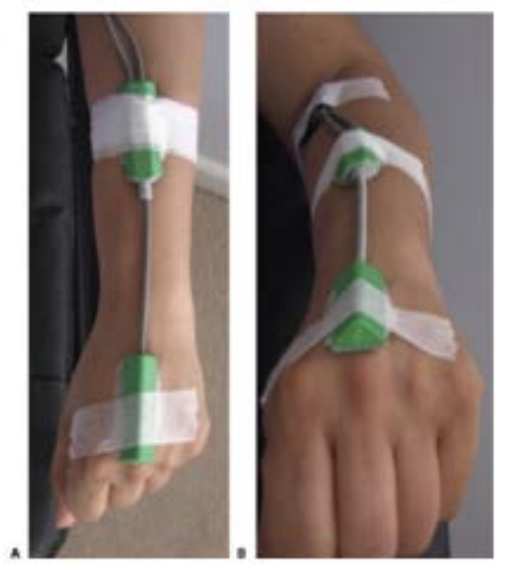

(b)

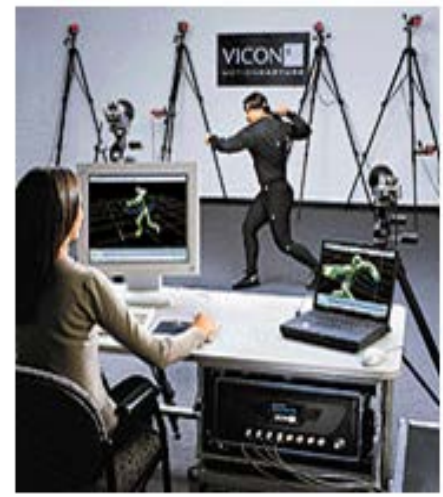

(d)

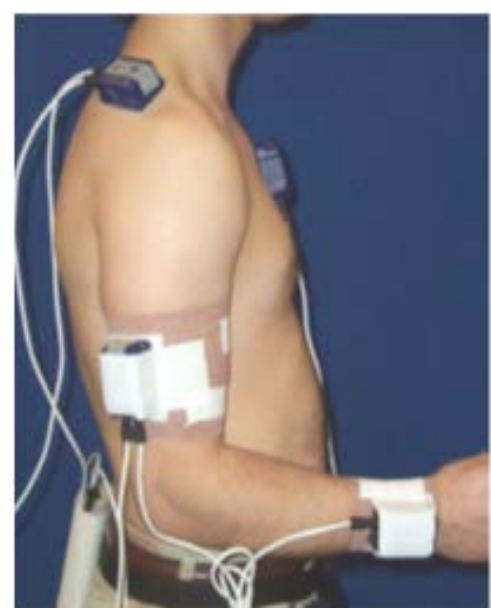

Figure 8: (a) A electromagnetic tracking system (Flock of Birds ${ }^{\mathrm{TM}}$, Ascension Technology Corp.); (b) An optoelectronic tracking system with markers on subject (Vicon ${ }^{\mathrm{TM}}$, Oxford Metrics Limited); (c) A electrogoniometer adhered to the forearm; and (d) Inertial measurement units attached to the body of a subject. 
Electrogoniometers are devices that can measure the relative angle between two segments. They are cheap and easy to use, but may not be able to accurately represent angles in 3-dimensions and may obstruct the movements in some configurations.

Most recent studies make use of either electromagnetic or optoelectronic systems in tracking of the arm motions. They are reliable, accurate, and many protocols have been developed to address operational issues. However, the costs and complexity in the implementation might be a hindrance for them to be a routine tool in a rehabilitation setting. The optoelectronic systems also have the additional problem of requiring an empty room of considerable size. One alternative to these systems which has gain popularity recently is the inertial measurement unit (IMU). Commercially available IMUs are relatively cheaper than both electromagnetic or optoelectronic systems and the costs of custommade units have the potential of going even lower. The angular measurements from an IMU usually are a result of fusion of data from gyroscopes, accelerometers and magnetometers. However, one drawback is its accuracy compared to the optoelectronic and electromagnetic systems which have many protocols proven to be effective. The solution to this is to develop algorithms that can apply the information captured by the IMUs onto accurate models of the human anatomy. And this shall be explained in details in the sections that follow. 


\subsection{The Denavit-Hartenberg (D-H) Representation of the Upper Limb Model}

The arm model developed in this study consists of a base (thorax), four segments (clavicle, scapula, humerus and forearm), and four joints (sternoclavicular (SC), acromioclavicular (AC), glenohumeral (GH), and elbow). Each of the first three joints (SC, AC and GH) has 3 DOFs, modeled by three orthogonal revolute joints, and the elbow joint has two DOFs, simulated by two non-orthogonal revolute joints. See Figure 9 for the illustration. The details on the shoulder and elbow models will be discussed in later sections. In this section, an overview of the arm model is presented.

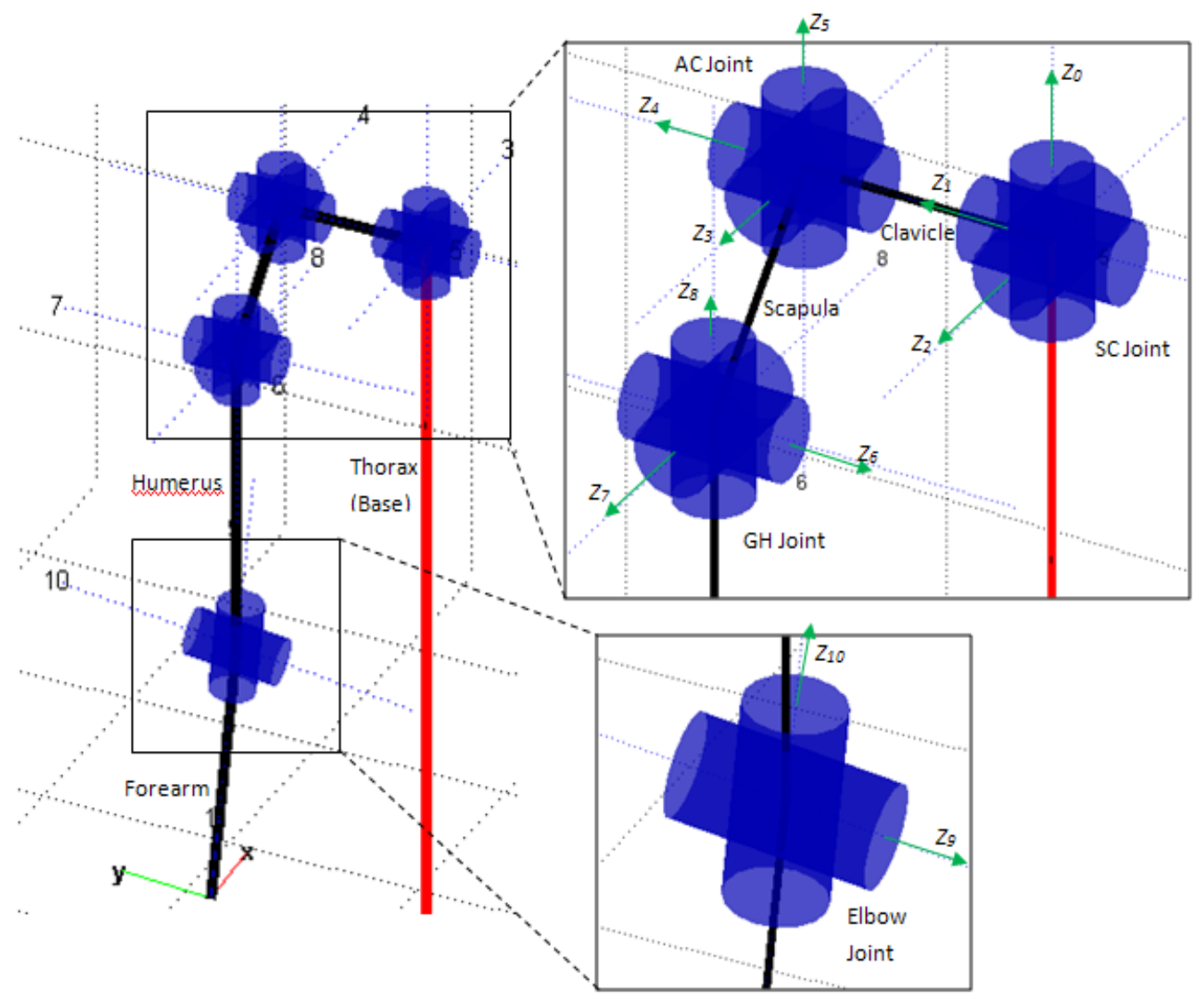

Figure 9: The illustration of the arm model that consists of 4 segments (clavicle, scapula, humerus and forearm), 4 joints (SC, AC, GH and Elbow), and a base (Thorax). The rotation axes, $Z_{n}$, in the D-H representation are also shown. 
There are altogether 11 rotation axes, $Z_{n},(n=0,1,2, \ldots, 10)$, assigned according to the D-H representation algorithm. The $\mathrm{D}-\mathrm{H}$ kinematic parameters $(\theta, d, a, \alpha)$ of each link are listed in Table 3.

Table 3: Rotation axis descriptions and D-H parameters

\begin{tabular}{|c|c|c|c|c|c|c|}
\hline Axis & Joint & $\begin{array}{c}\text { Description of the } \\
\text { rotation }\end{array}$ & $\boldsymbol{\theta}$ & $\boldsymbol{d}$ & $\boldsymbol{a}$ & $\boldsymbol{\alpha}$ \\
\hline 1 & SC & Protraction-Retraction & $\theta_{1}$ & 0 & 0 & $-\pi / 2$ \\
\hline 2 & SC & Axial Rotation & $\theta_{2}$ & 0 & 0 & $\pi / 2$ \\
\hline 3 & SC & Elevation-Depression & $\theta_{3}$ & 0 & $a_{3}$ & 0 \\
\hline 4 & AC & Medial-Lateral Rotation & $\theta_{4}$ & 0 & 0 & $-\pi / 2$ \\
\hline 5 & AC & Posterior-Anterior Tilt & $\theta_{5}$ & 0 & 0 & $-\pi / 2$ \\
\hline 6 & AC & AC Protraction-Retraction & $\theta_{6}$ & $d_{6}$ & $a_{6}$ & $-\pi / 2$ \\
\hline 7 & GH & $\begin{array}{c}\text { Posterior-Anterior } \\
\text { Elevation }\end{array}$ & $\theta_{7}$ & 0 & 0 & $-\pi / 2$ \\
\hline 8 & GH & Medial-Lateral Elevation & $\theta_{8}$ & 0 & 0 & $-\pi / 2$ \\
\hline 9 & GH & Axial Rotation & $\theta_{9}$ & $d_{9}$ & 0 & $\alpha_{9}$ \\
\hline 10 & Elbow & Flexion-Extension & $\theta_{10}$ & 0 & 0 & $\alpha_{10}$ \\
\hline 11 & Elbow & Supination-Pronation & $\theta_{11}$ & $d_{11}$ & 0 & 0 \\
\hline
\end{tabular}

The calculation of the link offsets ( $d_{6}, d_{9}$, and $\left.d_{11}\right)$ and the link lengths ( $a_{3}$ and $a_{6}$ ) will be explained in Section 3.5.5. The link twists ( $\alpha_{9}$ and $\alpha_{10}$ ) will be discussed in Section 3.3.5. The model is implemented using the Robotics Toolbox [101] in Matlab®. The following sections will discuss in details about the modeling of each segment of the arm.

\subsection{Placement of the Sensors}

The APDM Opal ${ }^{\mathrm{TM}}$ wireless inertial measurement units system is used in this study (APDM Inc., Portland, OR, USA). It has an accelerometer, a gyroscope, and a magnetometer all encased into a small unit of 48.4 x 36.5 x $13.4 \mathrm{~mm}$, and weighs $22 \mathrm{~g}$. The static and dynamic accuracy of the measured angle is $1.5^{\circ}$ and $2.8^{\circ}$ respectively. It is able to synchronize up to 24 units, with data synchronized typically within $1 \mathrm{~ms}$. The device local coordinate system, with $\mathrm{x}$-axis pointing downwards, y-axis pointing to the side, and z-axis pointing forwards, is with respect to an earth-based global coordinate system. The system calculates and gives the quaternion representation of the orientation as the output. In this study, data was acquired at a sampling rate of $128 \mathrm{~Hz}$. 
Four IMUs were attached to the subject's body. The first is positioned at the sternum, just below the neck, with the IMU frame $\left(x_{1}, y_{1}, z_{1}\right)$ shown in Figure 10. This IMU is used to measure the orientation of the thorax (which is expected to have very small changes throughout the experiment). The second IMU is attached to the broad, flat surface of the posterior-lateral acromion at the right shoulder, measuring the orientation of the scapula. The IMU frame is $\left(x_{2}, y_{2}, z_{2}\right)$. And the third IMU is strapped to the centre of the right upper arm to record the motion of the right humerus. The frame is designated as $\left(x_{3}, y_{3}, z_{3}\right)$. The fourth IMU is strapped to the lower arm near the wrist to measure movement of the forearm. The frame is designated as $\left(x_{4}, y_{4}, z_{4}\right)$. See

Table 4. The global coordinate system is defined as: $X_{G}-$ north, $Y_{G}-$ east, and $Z_{G}-$ down.

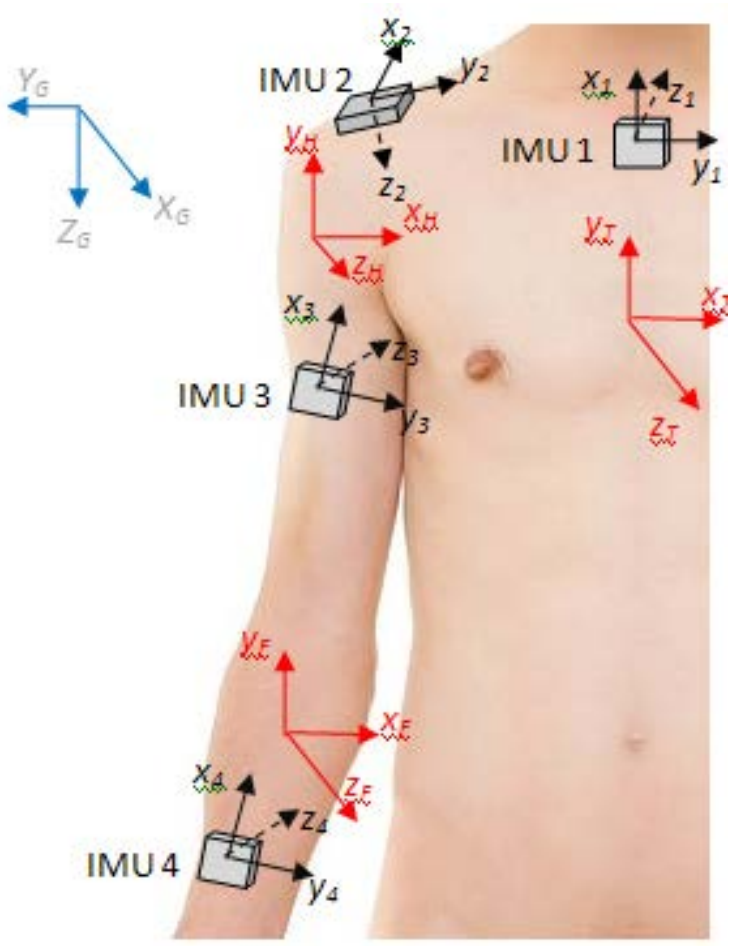

Figure 10: The coordinate systems and placement of the IMUs, the global frame, and the local frames. Note that the local frames of the clavicle and scapula are not shown. 
Table 4: Positions of IMUs and orientations of the frames

\begin{tabular}{|c|c|c|c|c|}
\hline IMU & Position & $\boldsymbol{x}$ & $\boldsymbol{y}$ & $\mathbf{z}$ \\
\hline 1 & $\begin{array}{c}\text { At the sternum, just below the } \\
\text { neck. }\end{array}$ & Cranial & $\begin{array}{c}\text { Lateral } \\
\text { Left }\end{array}$ & Dorsal \\
\hline 2 & $\begin{array}{c}\text { At the broad, flat surface of the } \\
\text { posterior-lateral acromion at } \\
\text { the right shoulder. }\end{array}$ & Dorsal & $\begin{array}{c}\text { Medial } \\
\text { Left }\end{array}$ & Caudal \\
\hline 3 & $\begin{array}{c}\text { At the centre of the right upper } \\
\text { arm. }\end{array}$ & Cranial & $\begin{array}{c}\text { Medial } \\
\text { Left }\end{array}$ & Dorsal \\
\hline 4 & At the lower arm near the wrist. & Cranial & $\begin{array}{c}\text { Medial } \\
\text { Left }\end{array}$ & Dorsal \\
\hline
\end{tabular}

In [90], the International Society of Biomechanics (ISB) proposed joint coordinate systems for each of the bones at the upper limb. The local coordinate systems of the thorax, scapula, clavicle, humerus and forearm are defined using anatomical landmarks, so to achieve standardization of joint motions. However, there are difficulties in the execution and instrumentation of locating these landmarks if the recording of the data is to be done in a non-laboratory environment. The locating of anatomical landmarks requires skilful palpation by trained therapists, which may not be readily available due to the low therapiststo-patients ratio in many ageing societies. The reliability and repeatability of palpation is also a concern [102]. The located landmarks have to be digitized using expensive and sophisticated camera systems or electromagnetic devices. The complexity and costs of these instruments may also hinder wide acceptance of the protocols in cost-sensitive fields like rehabilitation [98]. In view of these constraints, we propose to use local coordinate systems that are only dependent of the initial resting position of the arm. The local coordinates of the thorax, clavicle, scapula, humerus and forearm when the arm is resting naturally by the side are defined as: $x_{T}, x_{C}, x_{S}, x_{H}, x_{F}-$ medial (left), $y_{T}, y_{C}, y_{S}, y_{H}, y_{F}-$ cranial (up), and $z_{T}, z_{C}, z_{S}, z_{H}, z_{F}$ - ventral (front). With the help of a compass, the initial local 
$z$-axes were aligned with the north during the experiments, so that the orientations of the local systems are known with respect to the global system.

\subsection{The Shoulder Model}

\subsubsection{Introduction}

The human shoulder complex consists of three bones: clavicle, scapula and humerus, and with the thorax acting as a rigid base for the shoulder motions [103]. The kinematics of the shoulder are usually described by the movements at three joints: sternoclavicular (SC), acromioclavicular (AC) and glenohumeral (GH) joints $[84,90]$. The scapulothoracic articulation (ST), where the scapula glides over the rib cage, is sometimes considered to be a fourth joint, although it is not synovial [103, 104]. The SC, AC and GH joints all have three degrees of freedom (DOF), but the movements are constrained by the ST gliding plane and the conoid ligament between clavicle and scapula. Thus the scapula and clavicle plus the thorax form a closed-chain mechanism [88]. See Figure 11, taken from [105].

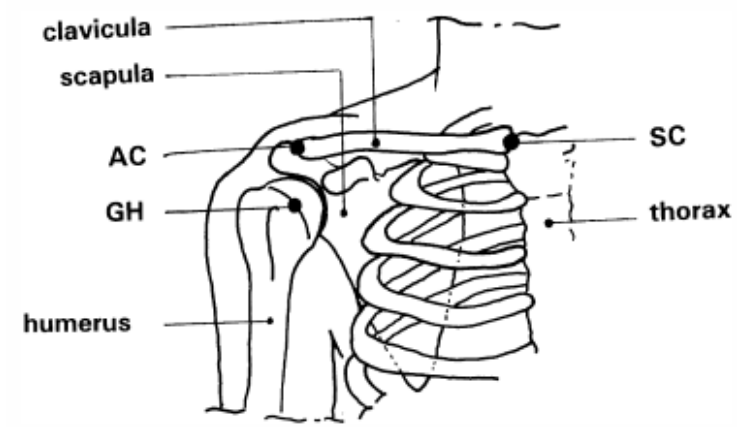

Figure 11: Anatomy of the shoulder. It consists of three bones: clavicle, scapula and humerus, and the thorax as the base. The three synovial joints are sternoclavicular (SC), acromioclavicular (AC) and glenohumeral (GH). The scapula glides along the ribcage forming the scapulothoracic articulation (ST).

The three-dimensional motion of the shoulder complex can provide important information in understanding abnormalities in upper limb motion. It is also crucial in the development of biomechanical models of the arm [106, 107]. Accurate measurement of the orientations of the scapula and clavicle are typically done either by tedious stationary posture recordings or in a highly constrained 
clinical environment using X-ray or complex camera systems [84]. However, these methods are not feasible when dynamic recordings are needed, e.g., in ergonomic and sports studies, and when the subjects are patients who have difficulties to comply with the protocols, e.g., in rehabilitation assessment of stroke patients.

An alternative method is to use wearable electromagnetic sensors or inertial measurement units (IMUs) attached to the skin to measure the orientations of the bones underneath $[98,107,108]$. Although these non-invasive methods have fewer constraints in terms of setups and executions, and can work in ambulatory situations, they are susceptible to the problems faced by every non-invasive method [84], like measurement noise and noise caused by soft tissue movements and involuntary rotations of other body joints by the subjects during experiments. The discrepancy in the measurements between invasive (where sensors are attached to pins drilled into the bones) and non-invasive methods becomes very large when the humeral elevation angle rises beyond $110^{\circ}$ [108].

Shoulder rhythm is the term used to describe the relationships between the motion of the humerus and scapula, and of the humerus and clavicle [109]. To address the issues on the difficulty of measuring scapular and clavicular movements, some studies have tried to establish models for shoulder rhythm through experiments. These models are useful in approximating the kinematics of large arm movements but they cannot capture subtle movements of the shoulder girdle like shrugging. Moreover, these models are based on the data collected from healthy subjects and might not be very accurate when apply on patients with shoulder abnormalities.

In our model, we propose a hybrid method that fuses the ambulatory data from the IMUs attached to the humerus and scapula, and the regression models based on the data collected from invasive measurements in [106].

\subsubsection{Shoulder Rhythm Model}

Invasive measurement methods, where sensors are fitted on pins that are securely drilled into the bones, can provide accurate measurements for the motions of the 
bones, especially for the scapula and clavicle. Although these methods are not practical to be used routinely in most applications, the data can be used as a benchmark for accuracy for non-invasive methods. In [106], the orientations of SC, AC and ST joints with respect to the elevation of GH joint are measured using a invasive method. The regression models of the graphs of SC and ST are used in this study as the shoulder rhythm models and are presented in Table 5 and Table 6. Only linear and quadratic functions are used in the regression models. No data was given for GH elevation angles less than $20^{\circ}$ in [106]. This may be due to the difficulty in handling ill-defined angles as a result of gimbal lock problem. In the regression models, we make use of the angles at the rest position and performed a linear fitting. The local coordinate systems at the joints in [106] are designated according to the recommendations given in [90], we mapped the angles to our designated frames using the direction cosines in the global reference frame given in [75].

Table 5: Regression models of sternoclavicular (SC) joint based on results in [106]. $\theta_{C X}, \theta_{C Y}$, and $\theta_{C Z}$ are the orientation of the clavicle with respect to the thorax in the $x_{C}, y_{C}$, and $z_{C}$ axes respectively. $\theta_{H}$ is the humeral elevation angle measured in the three different elevation planes $\left(90^{\circ}, 40^{\circ}, 0^{\circ}\right.$ with respect to the frontal plane). For $\theta_{C X}$, the variations are insensitive to the elevation plane. All angles are in degree.

\begin{tabular}{|c|c|c|c|c|c|c|c|c|c|}
\hline \multirow{2}{*}{$\begin{array}{c}\text { SC angles as } \\
\text { a function of } \\
\theta_{H} \text { in: }\end{array}$} & \multicolumn{3}{|c|}{$\theta_{C X}$} & \multicolumn{3}{|c|}{$\theta_{C Y}$} & \multicolumn{3}{|c|}{$\theta_{C Z}$} \\
\hline & $\theta_{H^{2}}$ & $\theta_{H}$ & const & $\theta_{H^{2}}$ & $\theta_{H}$ & const & $\theta_{H^{2}}$ & $\theta_{H}$ & const \\
\hline $\begin{array}{c}\text { Sagittal } \\
\text { Plane }\left(90^{\circ}\right) \\
\end{array}$ & \multirow{3}{*}{0.001} & \multirow{3}{*}{0.108} & \multirow{3}{*}{-2.91} & -0.001 & 0.082 & -21.87 & 0 & -0.049 & -8.902 \\
\hline $\begin{array}{c}\text { Scapular } \\
\text { Plane }\left(40^{\circ}\right) \\
\end{array}$ & & & & 0 & -0.128 & -20.7 & 0.001 & -0.097 & -9.322 \\
\hline $\begin{array}{c}\text { Frontal } \\
\text { Plane }\left(0^{\circ}\right)\end{array}$ & & & & 0.001 & -0.252 & -24.42 & 0.001 & -0.132 & -10.54 \\
\hline
\end{tabular}


Table 6: Regression models of scapulothoracic (ST) joint based on results in [106]. $\theta_{S X}, \theta_{S Y}$, and $\theta_{S Z}$ are the orientation of the scapula with respect to the thorax in the $x_{S}, y_{S}$, and $z_{S}$ axes respectively. $\theta_{H}$ is the humeral elevation angle measured in the three different elevation planes $\left(90^{\circ}, 40^{\circ}, 0^{\circ}\right.$ with respect to the frontal plane). For $\theta_{S X}$ and $\theta_{S Z}$, the variations are insensitive to the elevation plane. There no significant change in $\theta_{S Y}$ when the arm is abducted in the frontal plane. All angles are in degree.

\begin{tabular}{|c|c|c|c|c|c|c|c|c|c|}
\hline \multirow{2}{*}{$\begin{array}{l}\text { ST angles as a } \\
\text { function of } \theta_{H} \text { in: }\end{array}$} & \multicolumn{3}{|c|}{$\theta_{S X}$} & \multicolumn{3}{|c|}{$\theta_{S Y}$} & \multicolumn{3}{|c|}{$\theta_{S Z}$} \\
\hline & $\theta_{H^{2}}$ & $\theta_{H}$ & constant & $\theta_{H^{2}}$ & $\theta_{H}$ & $\begin{array}{c}\text { consta } \\
\text { nt }\end{array}$ & $\theta_{H^{2}}$ & $\theta_{H}$ & $\begin{array}{c}\text { consta } \\
\text { nt }\end{array}$ \\
\hline $\begin{array}{c}\text { Sagittal Plane } \\
\left(90^{\circ}\right)\end{array}$ & \multirow{3}{*}{0} & \multirow{3}{*}{-0.17} & \multirow{3}{*}{16.3} & -0.002 & 0.303 & 36.91 & \multirow{3}{*}{0} & \multirow{3}{*}{-0.33} & \multirow{3}{*}{-5.08} \\
\hline $\begin{array}{c}\text { Scapular Plane } \\
\left(40^{\circ}\right)\end{array}$ & & & & -0.001 & 0.072 & 36.73 & & & \\
\hline Frontal Plane $\left(0^{\circ}\right)$ & & & & 0 & 0 & 31 & & & \\
\hline
\end{tabular}

\subsubsection{Measurement of the orientations of scapula and humerus using IMUs}

The initial orientations of thorax relative to IMU 1, scapula relative to IMU 2 frame and humerus relative to IMU 3 frame are constant matrices given as

$$
\begin{aligned}
& R_{1 T}^{0}=\left(R_{G 1}^{0}\right)^{T} R_{G T}^{0} \\
& R_{2 S}^{0}=\left(R_{G 2}^{0}\right)^{T} R_{G S}^{0} \\
& R_{3 H}^{0}=\left(R_{G 3}^{0}\right)^{T} R_{G H}^{0}
\end{aligned}
$$

where the subscripts $G, 1,2,3, T, S$ and $H$ denote global, IMU 1, IMU 2, IMU 3, thorax, scapular and humeral frames respectively; the superscript 0 denotes initial position when the arm is resting by the side. The notation of the rotation matrix, $R_{X Y}$, represents orientation of frame $Y$ in frame $X$. Subsequently, the orientations of the thorax, scapula and humerus in the global frame during the arm motions can be calculated as

$$
\begin{aligned}
R_{G T} & =R_{G 1} R_{1 T}^{0} \\
R_{G S} & =R_{G 2} R_{2 S}^{0} \\
R_{G H} & =R_{G 3} R_{3 H}^{0}
\end{aligned}
$$


where $R_{G 1}, R_{G 2}$ and $R_{G 3}$ are the rotation matrices formed using the Euler angles given by the IMUs during the motions. Next, we can represent the scapular and humeral orientations in the thorax frame as

$$
\begin{aligned}
& R_{T S}=\left(R_{G T}\right)^{T} R_{G S} \\
& R_{T H}=\left(R_{G T}\right)^{T} R_{G H}
\end{aligned}
$$

According to the recommendations in [90], to obtain the Euler angles from the rotation matrices, $R_{T S}$ is decomposed using a Y-Z-X sequence ( $\theta_{S Y}^{m}, \theta_{S Z}^{m}$ and $\left.\theta_{S X}^{m}\right)$, and $R_{T H}$ is decomposed using Y-Z-Y sequence ( $\theta_{H}^{m}, \theta_{H P}^{m}$ and $\theta_{H A}^{m}$ ). The superscript $m$ differentiates the measured angles from the angles calculated from the regression models. The subscripts $P$ and $A$ in the humeral angles denote plane of elevation and axial rotation.

\subsubsection{Fusion of data from regression models and sensor measurements for scapular orientation}

The limitation of using IMU to measure scapular orientation becomes apparent when the humeral elevation angle is more than $100^{\circ}$. The sensor is incapable of capturing the real orientation of the bone when the arm movement is large due to the relative motions between the scapula and the skin. On the other hand, many of the regression models found in the literature $[84,109,110]$ present the scapular and clavicular orientations as a function of humeral elevation plane and angle. If an arm model solely relies on this humeral-scapular or humeral-clavcular relationship, it will be insensitive to shoulder movements like shrugging or involuntary upward rotation of the scapula and elevation of the clavicle, since the humerus may be stationary when these movements occur. This is undesirable in applications like rehabilitation where subtle differences in the movements at SC and AC joints between healthy subjects and patients are precisely the telltale signs a therapist may be looking for in assessing a therapeutic session.

To eliminate these problems, we propose a hybrid method that fuses the data from the sensors and the shoulder rhythm model from Ludewig et al. in [106]:

$$
\theta_{C}^{i}=\theta_{C}^{i-1}+(1-\alpha)\left(\theta_{M}^{i}-\theta_{M}^{i-1}\right)+\alpha\left(\theta_{R}^{i}-\theta_{R}^{i-1}\right)
$$


where $\theta_{M}^{i}, \theta_{R}^{i}$ and $\theta_{C}^{i}$ are the measured (from sensors), calculated (from rhythm model) and fused orientations at $i^{\text {th }}$ time step respectively; and $\alpha=\alpha\left(\Delta \theta_{H}^{m}\right)$ has a value between 0 and 1 , depending on the rate of change of the measured humeral elevation angle, $\Delta \theta_{H}^{m}$. See Figure 12 for the relationship between $\alpha$ and $\Delta \theta_{H}^{m}$. When the subject raises or lowers his arm, $\alpha$ has a value close to 1 and the fused orientation, $\theta_{C}^{i}$, will follow the rhythm model closely; and when the arm is near stationary, $\alpha$ will be near 0 , and the sensor data will be used. This hybrid method combines the accuracy of the rhythm model and the ability of the sensor to track subtle motions.

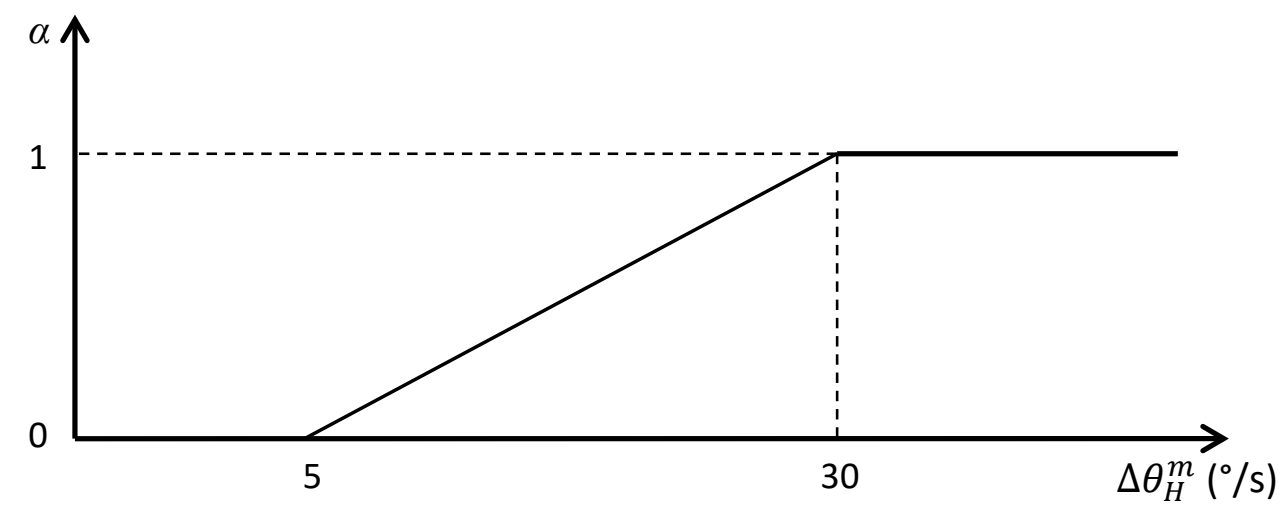

Figure 12: The relationship between $\alpha$ (in Eq. 3.9) and measured humeral elevation angle, $\Delta \boldsymbol{\theta}_{\boldsymbol{H}}^{\boldsymbol{m}}$.

\subsubsection{Validating Shoulder Model}

The orientation of the shoulder complex is difficult to measure directly with noninvasive methods. The shoulder rhythm model, on the other hand, was developed using measurements from invasive methods, and the orientation of the shoulder complex was accurately captured relative to the humeral elevation. For validation purpose, we propose to perform a simple experiment to demonstrate the working principles of the hybrid model, and compare the orientation profiles of simple motions with published results in [82]. 
Four healthy male subjects (mean age 26.5 years, with SD 2.65 years) volunteered for the experiment after informed consent was obtained from them. All subjects do not have any known upper limb disorder. The subjects are asked to perform five arm motions: (a) raise the arm from initial resting position to about $90^{\circ}$ and then to over the head in the sagittal plane at a controlled speed; (b) repeat the same motion as (a) but in the frontal plane; (c) shrugging of the shoulder without raising the arm; (d) moving the hand to grab a bottle of water and then towards the mouth as if drinking from the bottle and returned to the initial position; and (e) raise the hand to the forehead and towards the back of the head, as if combing the hair. The purpose of (a), (b) and (c) is to show the idea of the proposed method in two different situations: the former two are with significant humeral movements and the latter does not. The other two motions are the common activities of daily living (ADL), and the results can be used to compare with other upper limb kinematic studies [82].

Figure 13 shows the clavicular and scapular orientations of one subject performing arm motions (a) raising arm in the sagittal plane, (b) raising arm in the frontal plane, and (c) shrugging of shoulder. The solid lines are the fused orientations; the dotted lines are the orientations from the regression models; and the dot-dashed lines are the measured orientations from the IMUs. The continuous raising of the arm means that the rate of change of the elevation angle, $\Delta \theta_{H}^{m}$, is significant, and $\alpha$ is close to 1 , and as a result the fusion actions will favor the regression model. It can be seen clearly that for motions (a) and (b), the fused orientations followed closely to the regression models. When the elevation was about $90^{\circ}$ and maximum at about $150^{\circ}$, the arm was momentarily stationary, thus the measured data has more influences in these areas. This is a desirable characteristic of the hybrid method as it captures the subtle movements while the elevation angle remains unchanged. As a contrast, in Figure 13c, the subject performed a shrugging of the shoulder without lifting the arm. As the elevation angles changed only minimally, the regression models did not provide significant values for the clavicular and scapular angles. The value of $\alpha$ is near 0 and the 
fused angles largely followed the measured angles from the IMUs. The clavicle has relatively large variations in the pro-retraction $\left(\theta_{C Y}\right)$ and depression-elevation $\left(\theta_{C Z}\right)$ but not in the axial rotation $\left(\theta_{C X}\right)$. The scapula, on the other hand, has its all three rotations (tilting $\theta_{S X}$, pro-retraction $\theta_{S Y}$, and lateral/medial rotation $\theta_{S Z}$ ) showing significant variations during shrugging motions. This shows that the clavicle axial rotation is closely related to humeral elevation and not dependent on scapular motions, whereas the motions of the scapula is three dimensional. This observation is useful if the kinematic model is used in a dynamic model that includes musculoskeletal information. The movement of $\theta_{C X}$ can be an indicator to whether the muscles are working on elevating the humerus or just merely moving the scapula.

In Figure 14, the shoulder orientations of all four subjects performing the drinking and the combing motions are plotted with the movement cycle. Generally, all the angles from the subjects show similar patterns in their motions. We can compare the scapular orientations with the plots of measured scapular angles presented in [82]. The $\theta_{S Z}$ (lateral/medial rotation) for the drinking motion are very similar in both studies, with the angles rising gradually for about $15^{\circ}$ from the start till $50 \%$ of the movement cycle (the movement cycle in [82] corresponds to 50\% movement cycle in our experiment), as shown in Figure 14a. In [82], other two angles, $\theta_{S X}$ and $\theta_{S Y}$ (tilting and pro-retraction, respectively), were relatively flat. This is different from the observations in our experiment where both angles rose gradually and peaked at around $20^{\circ}$. The humeral elevation for the drinking motion ranged from $0^{\circ}$ to about $80^{\circ}$. For this range, according to the shoulder rhythm model, both $\theta_{S X}$ and $\theta_{S Y}$ would have peaked at around $20^{\circ}$. Similar situation occurred in the combing motion. In Figure $14 \mathrm{~b}$, the $\theta_{S Z}$ for the combing motion has good agreement between the two studies. But for $\theta_{S X}$ and $\theta_{S Y}$, the profiles presented in [82] are relatively flat again. The humeral elevation for the combing had an even larger range, from $0^{\circ}$ to $150^{\circ}$. The angular profiles for $\theta_{S X}$ and $\theta_{S Y}$ had a peak at around $35^{\circ}$ and $20^{\circ}$ respectively. The discrepancies can be explained by the fact that, in [82], the orientations of the 
scapula were measured using a single marker attached to the acromion, while we had made use of the hybrid model in our experiment. The former method of measurement is susceptible to inaccuracy due to soft tissue movement, as we have discussed previously, especially for movements that involve large humeral elevation. As the marker was attached to the flat part of the acromion, lateral rotation $\left(\theta_{S Z}\right)$ can be captured more accurately than tilting and pro-traction $\left(\theta_{S X}\right.$ and $\theta_{S Y}$, respectively). During the drinking and combing motions, the humerus was elevated substantially and continuously, so the hybrid model was following the shoulder rhythm model closely.

We showed that the proposed hybrid model is able to perform the intended mechanism: it follows the shoulder rhythm model when the humeral elevation is significant and continuous; or it adopts the measured scapular orientation by sensors attached to the acromion if there is little or no change in the humeral elevation angle; or a combination of both as described by Eq. 3.9. The hybrid model combines the strengths and eliminating the weaknesses of the shoulder rhythm model and the IMU sensor system. The orientations of scapula and clavicle calculated are used in the overall model of the arm. 

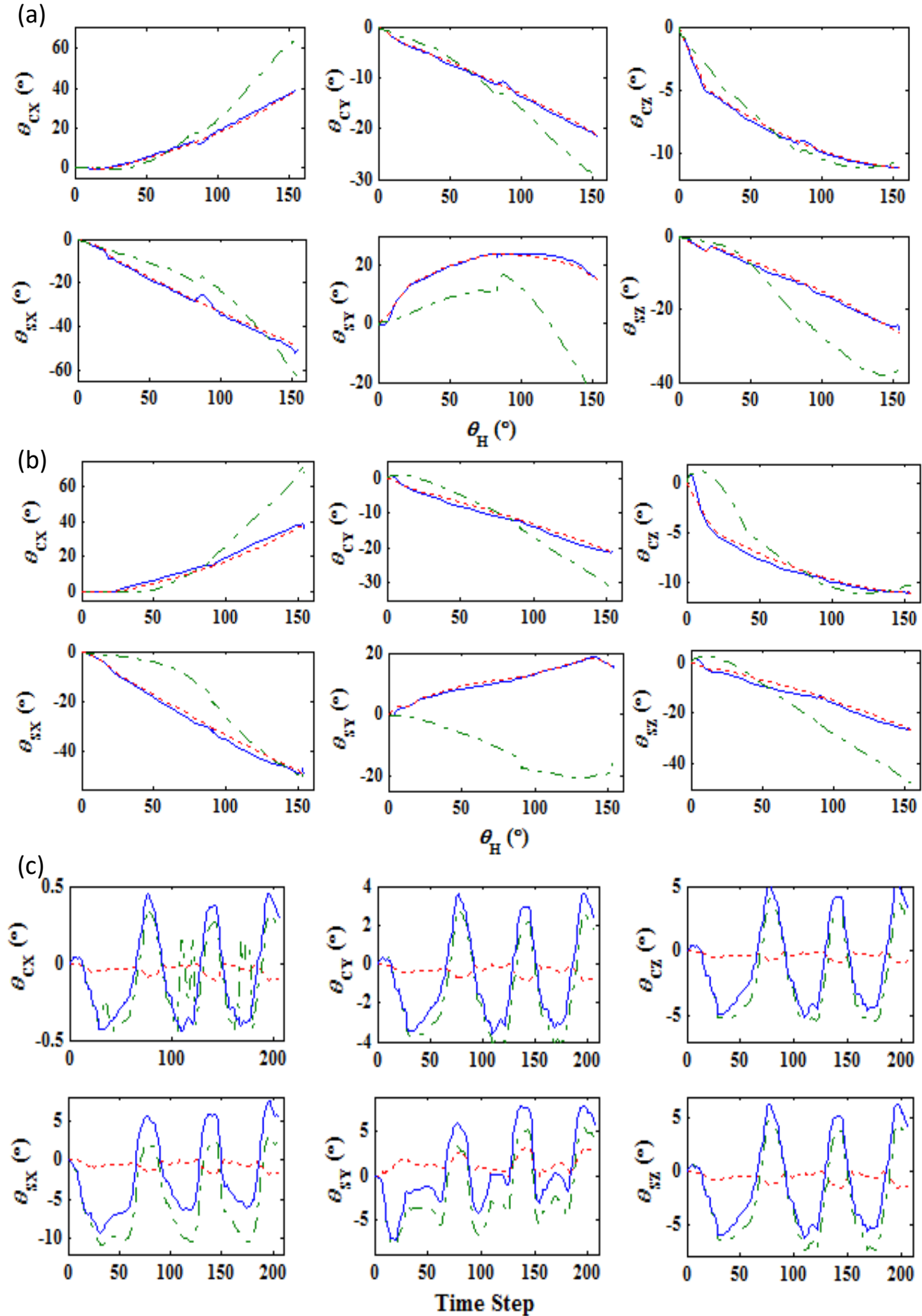

Figure 13: Clavicular ( $\theta_{C X}, \theta_{C Y}$, and $\left.\theta_{C Z}\right)$ and scapular $\left(\theta_{S X}, \theta_{S Y}\right.$, and $\left.\theta_{S Z}\right)$ orientations versus humeral elevation angle $\left(\theta_{H}\right)$ of one subject raising right arm in (a) the sagittal plane, and (b) the frontal plane; and (c) versus time-

step for shrugging. Solid lines are the fused angles; dotted lines are the regression model data; and dot-dashed lines are the measured data from the

IMUs. 
(a)
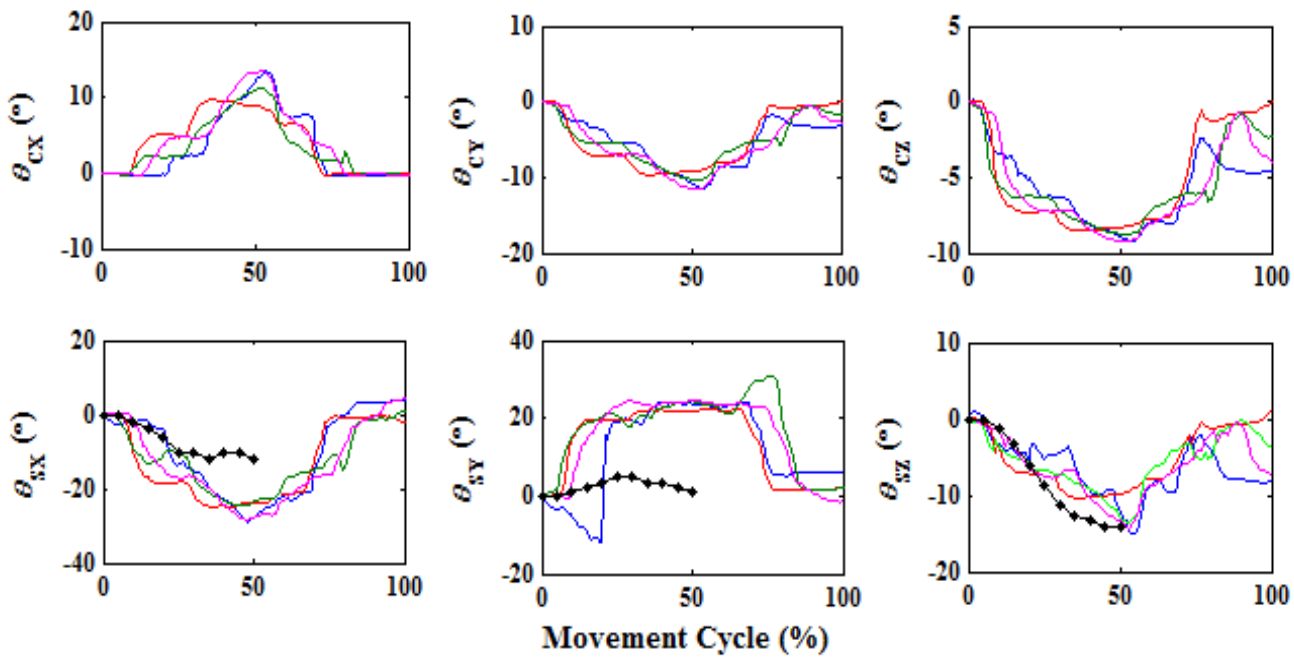

(b)
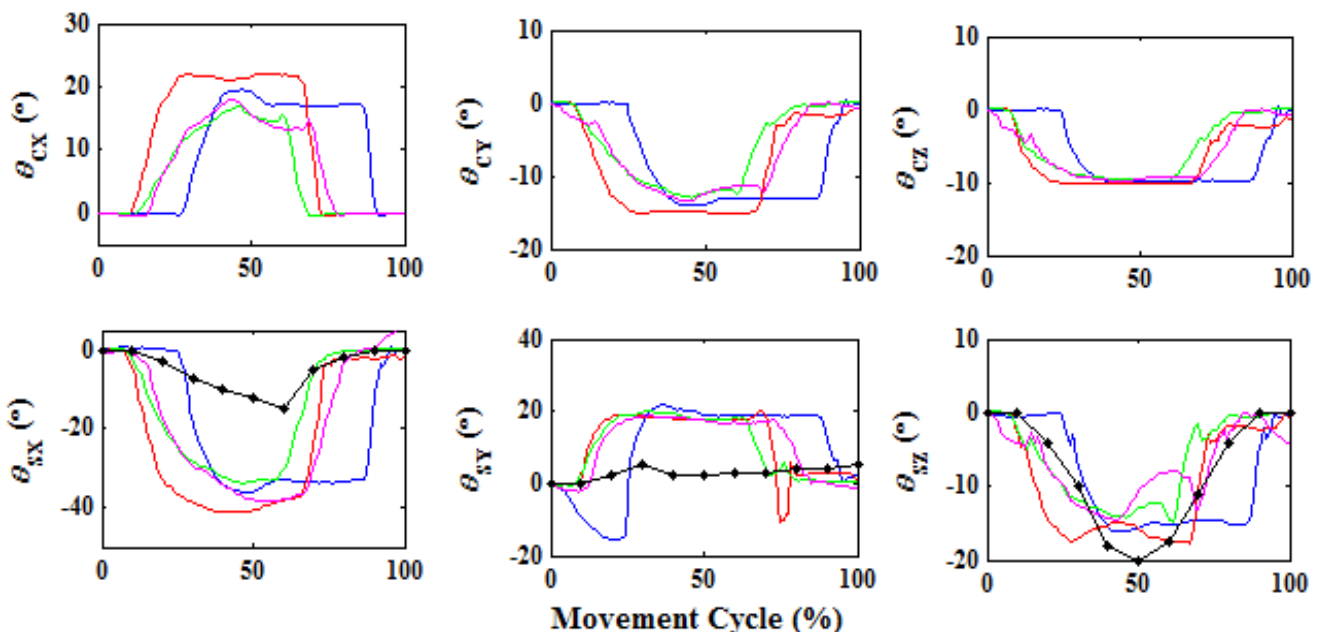

Figure 14: Clavicular $\left(\theta_{C X}, \theta_{C Y}\right.$, and $\left.\theta_{C Z}\right)$ and scapular $\left(\theta_{S X}, \theta_{S Y}\right.$, and $\left.\theta_{S Z}\right)$ orientations versus movement cycle in (a) drinking, and (b) combing motions of all four subjects (each colour represents one subject). The black lines are the corresponding angular profiles in [82] (for the drinking motion the cycle ends at $50 \%$ cycle of our experiment). No result of clavicular angles were presented in [82]. 


\subsection{The Elbow Model}

The methods to find the elbow rotation axes described in [111-113] required the precise locations of several bony landmarks with respect to a reference. These landmarks were either located using cadaver data or in vivo by using electromagnetic tracking devices with proper palpation techniques. The electromagnetic systems are too expensive and cumbersome for practical use of the kinematic data in a clinic or rehabilitation facility. And proper palpation requires the skills of trained therapists, which may not be readily available. The discrepancy in the definitions of palpated locations of the landmark among different therapists is also a problem.

In general, the flex/extension axis, $S_{F E}$, is not orthogonal to the longitudinal axis of the humerus, $y_{H}$. $S_{F E}$ and the pro/supination axis, $S_{P S}$, are also non-intersecting and not orthogonal to each other [103, 113]. This introduces a third angle, the carrying angle, when we want to obtain the rotation angles in $S_{F E}$ and $S_{P S}$ from decomposing the rotation matrix of the forearm relative to the upper arm. The definition of the carry angle is inconsistent [84], but in this context it is viewed as the angular offset between the orthogonal of $S_{F E}$ and $S_{P S}$. For the ease of finding the rotation angles in $S_{F E}$ and $S_{P S}$, the two axes are often taken to be orthogonal and the carrying angle is often conveniently regarded as a constant $[83,98]$. However, the carrying angle varies significantly among individuals [114], and it is dependent on the two elbow rotation angles [115]. In this study, we propose a method to decompose the rotation angles, obtained from the data captured by IMUs, in $S_{F E}$ and $S_{P S}$ directly using product of exponentials (POE) [116] and optimization without making any assumption on the carrying angle and the orthogonality of $S_{F E}$ and $S_{P S}$.

\subsubsection{Estimation of the rotation axes of the forearm}

To find $S_{F E}$ with respect to the humerus frame, the orientations of the IMUs strapped to the upper and lower arm between two consecutive positions were calculated. The orientation of the lower arm IMU in the upper arm IMU frame at the current (initial) position and the next (final) position are given by $[117,118]$ 


$$
\begin{aligned}
& R_{U L}^{i}=\left(R_{G U}^{i}\right)^{T} R_{G L}^{i} \\
& R_{U L}^{f}=\left(R_{G U}^{f}\right)^{T} R_{G L}^{f}
\end{aligned}
$$

where the superscripts $i$ and $f$ denote initial and final positions; the subscripts $G$, $U$ and $L$ denote global, upper arm IMU and lower arm IMU frames respectively. The rotation matrix $R_{X Y}$ represents orientation of frame $Y$ in frame $X$. The rotation matrix of the final position relative to the initial position in the lower arm IMU frame is

$$
R_{L}^{i f}=\left(R_{G L}^{i}\right)^{T} R_{G L}^{f}
$$

The skew-symmetric matrix, $W_{L}^{i f}$, that represents the rotation axis of $R_{L}^{i f}$ is [119]

$$
W_{L}^{i f}=R_{L}^{i f}-\left(R_{L}^{i f}\right)^{T}
$$

The rotation axis in the lower arm IMU frame is calculated as

$$
S_{L}=\left[w_{1}, w_{2}, w_{3}\right]^{T} / \sqrt{w_{1}^{2}+w_{2}^{2}+w_{3}^{2}}
$$

where $w_{1}, w_{2}$, and $w_{3}$ are the independent components of $W_{L}^{i f}$.

The rotation axis can be represented in the upper arm IMU frame using

$$
S_{U}=R_{U L}^{i} S_{L}
$$

And the rotation axis, when represented in the humerus frame, $H$, is

$$
S_{F E}=\left(R_{G H}^{i}\right)^{T} R_{G U}^{i} S_{U}
$$

The procedures to find $S_{P S}$ is exactly the same. In the first experiment, both $S_{F E}$ and $S_{P S}$ were calculated for ten times for the duration of the experiment. These axes are the Instantaneous Helical Axes (IHA) [120] during the motion of the elbow. The optimal axes were then calculated closest to the IHAs using leastsquared method as

$$
\text { Minimize } J\left(S_{\text {opt }}\right)
$$

where

$$
J\left(S_{\text {opt }}\right)=\sum_{i=1}^{10}\left\|S_{o p t}-S_{i}\right\|
$$

where $S_{i}$ are the IHAs in either flexion/extension or pronation/supination motions. 


\subsubsection{Decomposition of joint angles at the elbow}

After finding the orientations of the rotation axes of the forearm relative to the humerus, we can make use of the IMU data during arm motion to estimate the flexion/extension and pronation/supination angles of the elbow at each time-step. The orientations of the humerus frame in the upper arm IMU frame and the forearm frame in the lower arm IMU frame are constant matrices, given as

$$
\begin{aligned}
& R_{U H}^{0}=\left(R_{G U}^{0}\right)^{T} R_{G H}^{0} \\
& R_{L F}^{0}=\left(R_{G L}^{0}\right)^{T} R_{G F}^{0}
\end{aligned}
$$

where the superscript 0 denotes initial position when the arm is resting by the side. The orientations of the humerus and the forearm in the global frame at timestep $n$ can be calculated as

$$
\begin{aligned}
& R_{G H}^{n}=R_{G U}^{n} R_{U H}^{0} \\
& R_{G F}^{n}=R_{G L}^{n} R_{L F}^{0}
\end{aligned}
$$

Next, we can calculate the orientation of the forearm frame in the humerus frame as

$$
R_{H F}^{n}=\left(R_{G H}^{n}\right)^{T} R_{G F}^{n}
$$

The rotation matrix that represents the transformation of the forearm frame at time-step $n$ relative to $n-1$ in the humerus frame is

$$
R_{H}^{n-1, n}=\left(R_{H F}^{n-1}\right)^{T} R_{H F}^{n}
$$

The rigid body rotation about a fixed axis, $R \in \mathrm{SO}(3)$, where [119]

$$
\mathrm{SO}(3)=\left\{R \in \mathfrak{R}^{3 \times 3}: R R^{T}=I \text {, det } R=1\right\}
$$

The skew-symmetric matrix that corresponds to the axis of rotation is

$$
\widehat{W}=R-R^{T}=\left[\begin{array}{ccc}
0 & -w_{z} & w_{y} \\
w_{z} & 0 & -w_{x} \\
-w_{y} & w_{x} & 0
\end{array}\right]
$$

In the exponential form, $R=e^{\widehat{W} \theta}$, where $\theta \in \mathfrak{R}$ is the angle of rotation. In a more explicit form [116],

$$
R=e^{\widehat{W} \theta}=I+\sin \theta \frac{\widehat{W}}{\|W\|}+(1-\cos \theta) \frac{\widehat{W}^{2}}{\|W\|^{2}}
$$


In the context of the elbow joint which has two DOF, the joint motion can be represented by two consecutive rotations, one about $S_{F E}$ axis, followed by one about $S_{P S}$ axis:

$$
R_{\text {elbow }}=e^{\widehat{W}_{F E} \theta_{F E}} e^{\widehat{W}_{P S} \theta_{P S}}
$$

where $\widehat{W}_{F E}$ and $\widehat{W}_{P S}$ are the skew-symmetric matrices that correspond to $S_{F E}$ and $S_{P S}$ respectively; $\theta_{F E}$ and $\theta_{P S}$ are the respective rotation angles.

The rotation matrices $R_{H}^{n-1, n}$ and $R_{\text {elbow }}$ describe the same motion at the elbow if $\theta_{F E}$ and $\theta_{P S}$ are the change in angle about the respective axes from time-step n-1 to $n$. To calculate $\theta_{F E}$ and $\theta_{P S}$ from the rotation matrices without having to induce a third non-DOF angle (the carrying angle), we introduce an optimization routine:

\section{Minimize $J\left(\theta_{F E}, \theta_{P S}\right)$}

where

$$
J\left(\theta_{F E}, \theta_{P S}\right)=\sum_{i=1}^{3}\left\|e_{i} \times r_{i}\right\|^{2}
$$

where $r_{i}$ and $e_{i}$ are the $i^{\text {th }}$ column of $R_{H}^{n-1, n}$ and $R_{\text {elbow }}$ respectively. The optimization routine ensures the respective column vectors of $R_{H}^{n-1, n}$ and $R_{\text {elbow }}$ are optimally in the same directions. We do not attempt to match all nine components of the matrices because it may cause convergence issues in the optimization process. The routine is implemented in Matlab ${ }^{\circledR}$ using the function fsolve with the 'trust-region-dogleg' algorithm.

\subsubsection{Validating the elbow model}

There were two sets of experiment done in this study. The first was to find the two axes of rotation of the forearm $\left(S_{F E}\right.$, flexion-extension, and $S_{P S}$, pronationsupination) with respect to the humerus frame. As the sensors were wireless, the subjects were able to stand at a clearing in the lab, away from any metallic objects that might affect the measurements. To find $S_{F E}$, the subject stood upright with both arms resting naturally by the sides. During the experiment, the subject flexed the forearm completely at a controlled speed, and then extended back to the initial position. To find $S_{P S}$, the subject fully pronated the forearm from the initial 
position, and then supinated the forearm fully at a controlled speed. Both motions were performed three times by the subjects.

The second experiment was designed to test the accuracy of the methods proposed in this study. The subjects stood upright with their arms resting naturally by the sides. Then they performed the following two motions: (1) raising the whole arm in the sagittal plane to above the head at a controlled speed; (2) moving the hand towards the mouth as if drinking water from a cup and returned to the initial position.

Four healthy male subjects (mean age 26.5 years, with SD 2.65 years) volunteered for the experiment after informed consent was obtained from them. All subjects do not have any known upper limb disorder.

The calculated $S_{F E}$ and $S_{P S}$ of one subject are shown in Figure 15. The dashed lines show the axes of rotation of the twenty segments throughout the duration of the experiment. The solid lines are the optimal axes. The view is presented in the frontal plane where the relative orientation between $S_{F E}$ and $S_{P S}$ is most apparent. The results of all four subjects are shown in Table 7. The error estimations of the optimal axes are calculated as [112]:

$$
e_{S}=\frac{1}{N} \sum_{i=1}^{N} \cos ^{-1}\left(S_{o p t} \cdot S_{i}\right)
$$

where $S_{i}$ are the IHAs in either flexion/extension or pronation/supination motions, and $S_{\text {opt }}$ is the optimal axes. 


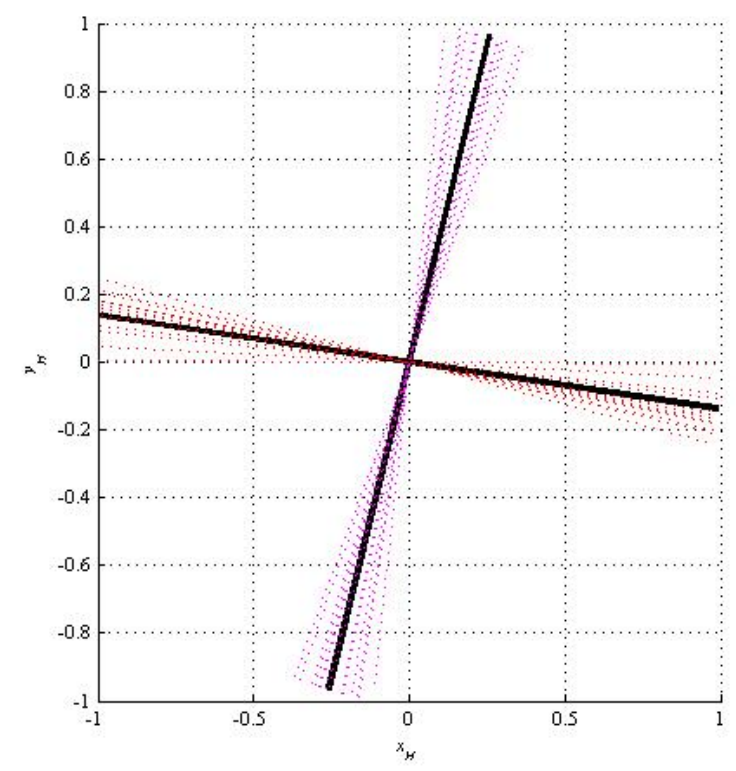

Figure 15: The calculated $S_{F E}$ and $S_{P S}$ of one subject shown in the frontal plane. The dashed lines are the axes calculated between two consecutive positions. The solid lines are the optimal axes. The axes are dimensionless as only the directions of the axes are plotted.

Table 7: Calculated $S_{F E}$ and $S_{P S}$

\begin{tabular}{|c|c|c|c|c|c|c|c|c|}
\hline \multirow{2}{*}{ Subject } & \multicolumn{4}{|c|}{$S_{F E}$} & \multicolumn{4}{|c|}{$S_{P S}$} \\
\hline & $x_{\text {opt }}$ & Yopt & $z_{\text {opt }}$ & $e_{s}(\underline{o})$ & $x_{\text {opt }}$ & $y_{o p t}$ & $z_{\text {opt }}$ & $e_{s}(\underline{o})$ \\
\hline 1 & 0.968 & -0.229 & -0.103 & 5.45 & 0.274 & 0.957 & -0.097 & 4.39 \\
\hline 2 & 0.918 & -0.251 & -0.307 & 6.00 & 0.240 & 0.968 & -0.073 & 3.45 \\
\hline 3 & 0.996 & -0.087 & -0.035 & 8.77 & 0.176 & 0.984 & -0.034 & 2.09 \\
\hline 4 & 0.987 & -0.151 & 0.058 & 5.24 & 0.103 & 0.985 & -0.132 & 2.28 \\
\hline Mean $(s d)$ & $\begin{array}{c}0.967 \\
(0.035) \\
\end{array}$ & $\begin{array}{c}-0.180 \\
(0.075) \\
\end{array}$ & $\begin{array}{c}-0.097 \\
(0.155) \\
\end{array}$ & $\begin{array}{c}6.37 \\
(1.64) \\
\end{array}$ & $\begin{array}{c}0.198 \\
(0.075) \\
\end{array}$ & $\begin{array}{c}0.974 \\
(0.014) \\
\end{array}$ & $\begin{array}{c}-0.084 \\
(0.041) \\
\end{array}$ & $\begin{array}{c}3.05 \\
(1.08) \\
\end{array}$ \\
\hline Ref [112] & $\begin{array}{c}0.965 \\
(0.035) \\
\end{array}$ & $\begin{array}{c}-0.134 \\
(0.032) \\
\end{array}$ & $\begin{array}{c}-0.212 \\
(0.118) \\
\end{array}$ & $\begin{array}{c}4.12 \\
(0.670) \\
\end{array}$ & & & & \\
\hline $\operatorname{Ref}[120]$ & $\begin{array}{c}0.992 \\
(0.007)\end{array}$ & $\begin{array}{c}-0.084 \\
(0.066)\end{array}$ & $\begin{array}{c}-0.018 \\
(0.083)\end{array}$ & $\begin{array}{c}4.72 \\
(0.073)\end{array}$ & $\begin{array}{c}0.02 \\
(0.088)\end{array}$ & $\begin{array}{c}0.996 \\
(0.004)\end{array}$ & $\begin{array}{c}-0.022 \\
(0.027)\end{array}$ & $\begin{array}{c}4.59 \\
(0.039)\end{array}$ \\
\hline
\end{tabular}

The average optimal axes and error estimations of $S_{F E}$ and $S_{P S}$ of all the subjects in the humerus frame are $[0.967,-0.180,-0.097]$ and $6.37^{\circ}$, and $[0.198,0.974$, 0.084 ] and $3.05^{\circ}$ respectively. The results from [112] and [120] are included for comparison. The axes calculated and the corresponding errors in this study are very similar to that published in [112]. The axes calculated in [120] are closer to 
the axes in the humerus frame. This may be due to the fact that the results were calculated from cadaveric data where the sensors were fixed directly to the bones. Whereas in living subjects there are always some disturbances caused by soft tissue movements and involuntary motions from other parts of the body, there are no such issues in cadavers.

The trajectories of the flexion/extension angle, $\theta_{F E}$ and the pronation/supination angle, $\theta_{P S}$ of a subject performing the arm motions are presented in Figure 16. For Figure 16a and b, the subject flexed his arm fully from initial position. The $\theta_{F E}$ started from $0^{\circ}$ and decreased steadily to $-118^{\circ}$. As the subject was told to flex the arm naturally, the forearm supinated slightly during theflexion.

The subject pro/supinated the forearm from the initial natural resting position in the second motion, and the angles are shown in Figure 16c and $\mathrm{d}$. The range of the $\theta_{P S}$ is from $-50^{\circ}$ to $50^{\circ}$. As no external constraint was used on the subject's body during the experiment, the arm flexed slightly during movement. The subject raised his arm from initial position to above his head. As expected, both angles have only small variations during this movement, as shown in Figure 16e and $\mathrm{f}$. In the last movement, the subject was told to pick up a bottle from a table in front of him, drink from it, and put it back. The $\theta_{F E}$ nearly reached its maximum at about $-100^{\circ}$ as shown in Figure $16 \mathrm{~g}$. It is apparent from Figure $16 \mathrm{~h}$ that the movement invoked mainly pronation.

All subjects have similar ranges and patterns in $\theta_{F E}$ and $\theta_{P S}$ in the first three movements, but not in the drinking motion, as shown in Figure 17. This is due to the differences in the trajectory taken by each subject in more complicated movements. 
(a)

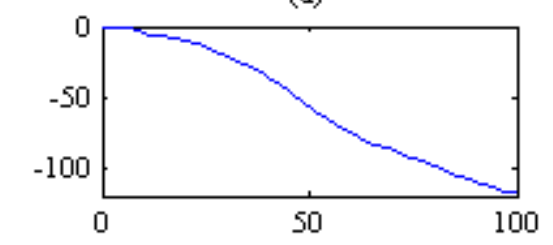

(c)

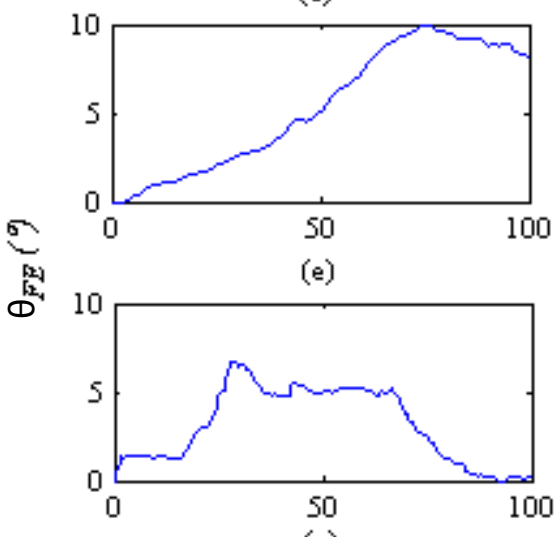

(g)

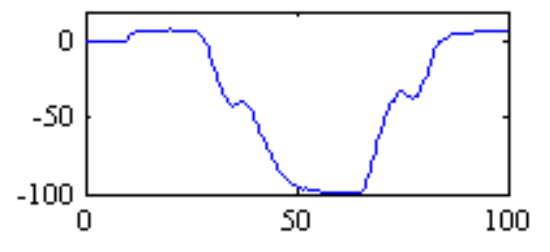

movement cycle (\%) (b)

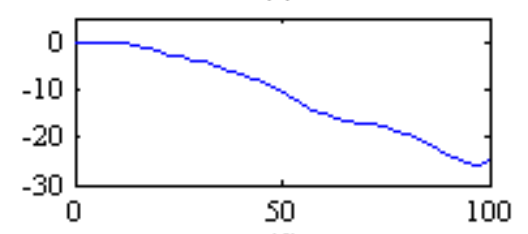

(d)

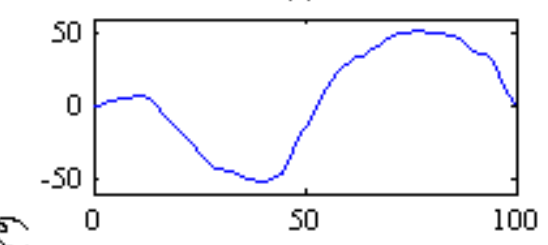

(f)

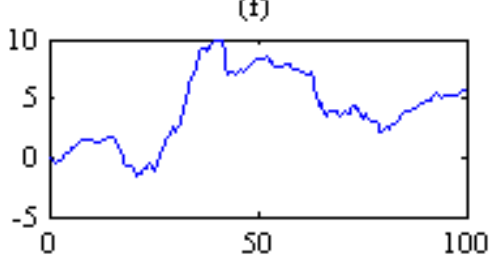

(h)

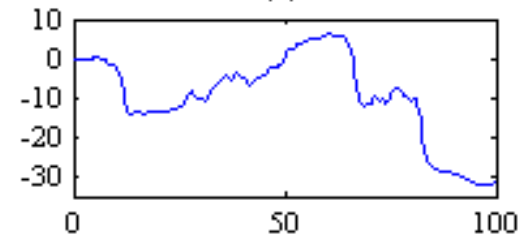

Figure 16: The plot of the trajectories of $\theta_{F E}$ (left column) and $\theta_{P S}$ (right column) versus the percent of movement cycle of one subject: (a) \& (b) flexion; (c) \& (d) supination/pronation ; (e) \& (f) arm raised in the sagittal plane; and (g) \& (h) drinking water. 
(a)

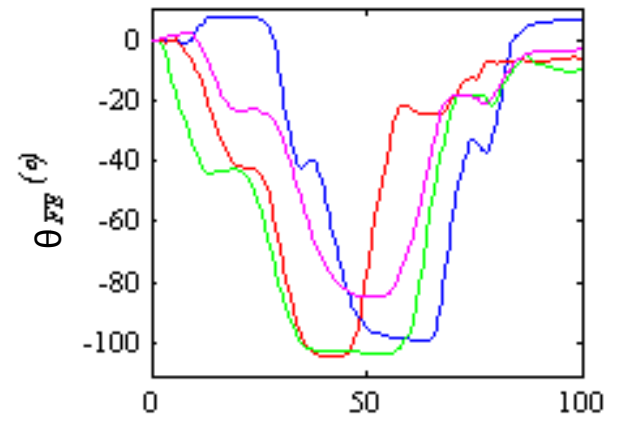

(b)

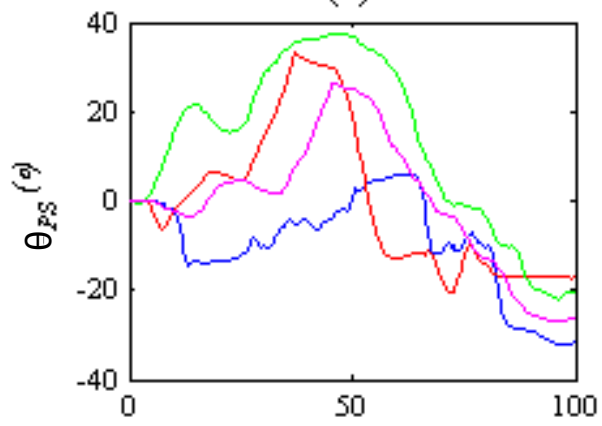

movement cycle (\%)

Figure 17: Comparison of (a) $\theta_{F E}$ and (b) $\theta_{P S}$ during the drinking movement among subjects.

The accuracy of obtaining the flexion/extension angle, $\theta_{F E}$ and the pronation/supination angle, $\theta_{P S}$ using the optimization method with respect to the measurements by the sensors is tested by an error estimation [116]. We define the logarithm of a rotation matrix, $R \in \mathrm{SO}(3)$, as

$$
\log [R]=\frac{\phi}{2 \sin \phi}\left(R-R^{T}\right)
$$

where $2 \cos \phi=\operatorname{trace}(R)-1$, and $\log [R]$ is skew-symmetric. The average quantified orientational deviations between the calculated and measured poses is defined as

$$
\Delta R=\frac{1}{m} \sum_{i=1}^{m}\left\|\log \left(R_{m i}^{-1} R_{c i}\right)^{\vee}\right\|
$$

where $m$ is the number of time-steps, $R_{m}$ is the measured rotation matrix (in this case $R_{H}^{n-1, n}$ ), and $R_{C}$ is the calculated matrix (in this case $R_{\text {elbow}}$ ). The notation $(\cdot)^{\vee}$ refers to the $\mathfrak{R}^{3}$ vector that represents $\log [R]$. The results of $\Delta R$ of each subject performing each movement are shown in Table 8. It can be seen that the deviations are small, and this means that the optimization gives reliable results. 
Table 8: Orientational deviation between the calculated and measured poses, $\Delta R$

\begin{tabular}{|c|c|c|c|c|}
\hline \multirow{2}{*}{ Subject } & \multicolumn{4}{|c|}{$\Delta \boldsymbol{R}\left(\mathbf{(}^{\mathbf{}}\right.$} \\
\cline { 2 - 5 } & Flexion & Pro/Supination & Raise Arm & Drinking \\
\hline 1 & 0.756 & 0.344 & 0.141 & 0.250 \\
\hline 2 & 2.05 & 0.412 & 0.303 & 0.604 \\
\hline 3 & 2.26 & 0.571 & 0.603 & 0.557 \\
\hline 4 & 0.622 & 0.101 & 0.194 & 0.527 \\
\hline \multirow{2}{*}{ Mean $(s d)$} & $1.42(0.853)$ & $0.357(0.195)$ & $\begin{array}{c}0.310 \\
(0.207)\end{array}$ & $0.485(0.160)$ \\
\hline
\end{tabular}

The method described in this chapter demonstrates that finding elbow rotation angles using IMUs can be performed easily and with accuracy comparable to protocols that requires palpation and more expensive devices. It also avoids complex and costly minimization computations that involve partial derivatives and integrals [121]. Comparing the errors in the calculations of $S_{F E}$ and $S_{P S}$ with [112] and [120], we see that the proposed method yields reasonably accurate results.

The mean angle between $S_{F E}$ and $S_{P S}$ among the four subjects is $88.6^{\circ}$ with a standard deviation of $3.67^{\circ}$. This justifies our choice of not using Euler decomposition which assumes orthogonality of the two axes to find $\theta_{F E}$ and $\theta_{P S}$. The effect of the non-orthogonality of $S_{F E}$ and $S_{P S}$ will be more apparent if the method is used in a whole arm model that aims to estimate muscle strengths. In these types of complex musculoskeletal model, the lines of action of the muscles with respect to the joints are very important. The assumption of orthogonality will compromise the accuracy in these models.

The optimization routine used in calculating $\theta_{F E}$ and $\theta_{P S}$ yields results that have very small deviation from the measured data. The cost of computation is low since there are only two variables. This eliminates the need to introduce the carrying angle which is not a DOF at the elbow. In the complete arm model, $\theta_{F E}$ and $\theta_{P S}$ calculated using the method can be viewed as the joint angles of the elbow which can be modeled by two revolute joints. 


\subsubsection{Calculating the link twists $\left(\alpha_{9}\right.$ and $\left.\alpha_{10}\right)$}

The link twists $\left(\alpha_{9}\right.$ and $\left.\alpha_{10}\right)$ are not multiples of $\pi / 2$ because of the nonorgothonality of $S_{F E}$ and $S_{P S}$, and have to be computed separately. The vectors representing the two axes are calculated in the humeral frame. The link twist is defined as the angle between two consecutive axes of joints, $Z_{k-1}$ and $Z_{k}$, about $X_{k}$ axis. In the arm model, $\alpha_{9}$ is the angle between $Z_{8}$ and $Z_{9}$, and $\alpha_{10}$ is between $Z_{9}$ and $Z_{10}$. See Figure 18 .

In the context of the D-H model, $S_{F E}$ is $Z_{9}$ and $S_{P S}$ is $Z_{10}$, and $\alpha_{9}$ and $\alpha_{10}$ are computed as

$$
\begin{gathered}
\alpha_{9}=\cos ^{-1} \frac{Z_{8} \cdot Z_{9}}{\left|Z_{8}\right|\left|Z_{9}\right|}=\cos ^{-1} Z_{8} \cdot Z_{9} \\
\alpha_{10}=\cos ^{-1} \frac{Z_{9} \cdot Z_{10}}{\left|Z_{9}\right|\left|Z_{10}\right|}=\cos ^{-1} Z_{9} \cdot Z_{10}
\end{gathered}
$$
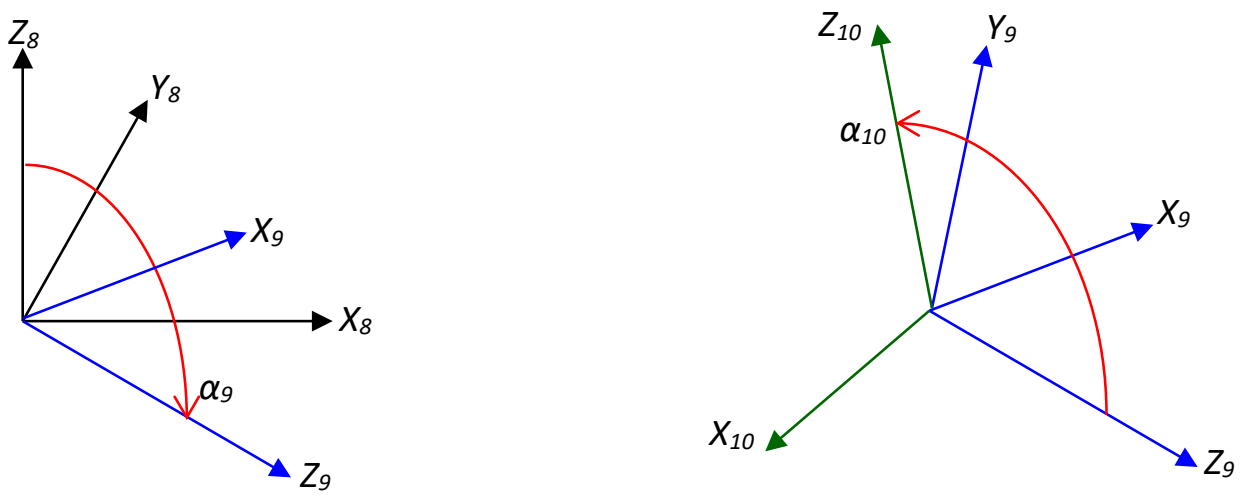

Figure 18: The definitions of the link twist $\alpha_{9}$ and $\alpha_{10}$. 


\subsection{Anthropometric Data}

The calculation of joint torque requires the length, mass, center of mass and the inertial properties of each segment in the model. Obtaining accurate anthropometric information from subjects is a tedious and difficult task, for example, in [122], the body segment inertial parameters (BSIPs: mass, center of mass and principal radii of gyration) of the subjects are obtained using a gammaray scanner. These methods are too expensive, too complex or too invasive to be used in a rehabilitation setting. Many researchers thus compromised the accuracy for convenience and cost by using scaled estimations from published anthropometric data [123].

The anthropometric information of the shoulder complex is very scarce in the literature, and data that separate clavicle from the scapula is even rarer. In [110], the scapula and the clavicle are assumed to have zero mass and inertia when their main purpose was to estimate forces at the glenohumeral joint. This is undesirable in our model because the two bones are two separate segments and their masses and inertias are significant factors in the calculation of the joint torques.

In our model, we make use of the BSIPs of the clavicle and scapula in [75], which presented the data of the two bones separately. However, the data is based on the measurements of a single cadaver. This can be used together with the data in [67] where a table of BSIP data from various sources is compiled. It is useful because the parameters are calculated as a percentage of body mass and segment lengths, which are measurable.

\subsubsection{Measurable Parameters}

In the arm model, four parameters that can be easily measured are used as inputs to scale the BSIP data. They are (a) body mass, (b) length of the clavicle, (c) length of the humerus, and (d) length of the forearm. The length of the clavicle, $L_{C}$, of the subject is measured using a measuring tape from the Sternoclaviculare (SC) just beside Incisura Jugularis (IJ) at the thorax to the Acromioclaviculare (AC). The length of the humerus, $L_{H}$, is measured from approximate position of 
Glenohumeral joint (GH) to the medial epicondyle (EM) at the elbow. And the length of the forearm, $L_{F}$, is measured from EM to the wrist. All of the points can be easily palpated by an untrained person.

\subsubsection{Mass of segments}

The mass of the cadaver, $M_{B}$, used in [75] is not stated in the paper. However, based on the mass of humerus $(2.16 \mathrm{~kg})$ stated, we can make use of the table in [67] to do an estimate:

$$
M_{B}=\frac{2.16}{0.028}=77 \mathrm{~kg}
$$

$M_{B}$ will be our base mass and the mass of the subject performing the experiment is denoted as $M_{T}$. The mass of the clavicle and scapula given in [75] is 0.12784 $\mathrm{kg}$ and $0.94898 \mathrm{~kg}$ respectively. Therefore, the clavicular mass, $M_{C}$, and the scapular mass, $M_{S}$, of the subject are scaled as:

$$
\begin{aligned}
& M_{C}=0.12784 \times \frac{M_{T}}{77} \mathrm{~kg} \\
& M_{S}=0.94898 \times \frac{M_{T}}{77} \mathrm{~kg}
\end{aligned}
$$

The humeral mass, $M_{H}$, and the mass of the forearm, $M_{F}$, are calculated using data in [67]:

$$
\begin{aligned}
& M_{H}=0.028 \times M_{T} \mathrm{~kg} \\
& M_{F}=0.022 \times M_{T} \mathrm{~kg}
\end{aligned}
$$

\subsubsection{Center of mass (COM) of segments}

Based on the table that lists the coordinates of the bony landmarks in [75], the length of the clavicle of the cadaver (SC to AC) is $0.154 \mathrm{~m}$. We will use the ratio of $L_{C}$ to this length to scale the positions of COM of clavicle and scapula of a subject. Based on the same table, the coordinates of the COM of clavicle of the cadaver, with respect to SC is:

$$
\left[X_{C B}, Y_{C B}, Z_{C B}\right]=[0.04876,0.545,-0.0175]
$$

And the coordinates of the COM of scapula, with respect to AC is:

$$
\left[X_{S B}, Y_{S B}, Z_{S B}\right]=[-0.087,0.053,0.02596]
$$

Thus, the coordinates of the COM of clavicle and scapula of a subject are scaled to be: 


$$
\begin{aligned}
{\left[X_{C}, Y_{C}, Z_{C}\right] } & =\frac{L_{C}}{0.154}\left[X_{C B}, Y_{C B}, Z_{C B}\right] \\
{\left[X_{S}, Y_{S}, Z_{S}\right] } & =\frac{L_{C}}{0.154}\left[X_{S B}, Y_{S B}, Z_{S B}\right]
\end{aligned}
$$

The COM of humerus and forearm are calculated using data in [67] as, respectively:

$$
\begin{aligned}
& {\left[X_{H}, Y_{H}, Z_{H}\right]=\left[0,0,-0.436 L_{H}\right]} \\
& {\left[X_{F}, Y_{F}, Z_{F}\right]=\left[0,0,-0.682 L_{F}\right]}
\end{aligned}
$$

\subsubsection{Moments of inertial (MOI) of segments}

The MOI of clavicle and scapula of the cadaver in the three principal axes from [75] in the respective link frames are:

$$
\begin{array}{cc}
{\left[I_{X C B}, I_{Y C B}, I_{Z C B}\right]=[0.0011,0.00039,0.00123] \mathrm{kgm}^{2}} & \text { Eq.( } 3.45) \\
{\left[I_{X S B}, I_{Y S B}, I_{Z S B}\right]=[0.0191,0.0112,0.0301] \mathrm{kgm}^{2}} & \text { Eq.( } 3.46)
\end{array}
$$

The MOI of clavicle of a subject is scaled using parallel-axis theorem and the thin cylinder formula as:

$$
\left[I_{X C}, I_{Y C}, I_{Z C}\right]=\frac{M_{T} L_{C}^{2}}{77(0.154)^{2}}\left[I_{X C B}, I_{Y C B}, I_{Z C B}\right] \mathrm{kgm}^{2}
$$

The MOI of scapula is scaled using only the mass as:

$$
\left[I_{X S}, I_{Y S}, I_{Z S}\right]=\frac{M_{T}}{77}\left[I_{X S B}, I_{Y S B}, I_{Z S B}\right] \mathrm{kgm}^{2}
$$

The MOI in the two non-axial principal axes of the humerus of a subject is calculated using data from [67] and they are assumed to be the same:

$$
I_{X H}=I_{Y H}=\left(0.322 L_{H}\right)^{2} M_{H} \mathrm{kgm}^{2}
$$

The MOI in the axial axis is scaled using data from [75]:

$$
I_{Z H}=0.00392 \frac{M_{T}}{77} \mathrm{kgm}^{2}
$$

For the forearm, the computation of the MOI in the two non-axial principal axes uses data in [67]:

$$
I_{X F}=I_{Y F}=\left(0.468 L_{F}\right)^{2} M_{F} \mathrm{kgm}^{2}
$$

The information for the MOI in the axial axis is divided into two segments, the ulna and radius. However, we see that the MOI in the axial axis of both segments are nearly the same at $15.44 \%$ of the MOI of the non-axial axes. We use this fact to compute $I_{Z F}$ : 


$$
I_{Z F}=0.154 I_{X F} \mathrm{kgm}^{2}
$$

\subsection{Computation of the Joint Torques}

After computing the joint angles at each time-step for the duration of the movement, we need to compute the joint angular velocities and accelerations before the joint torques can be found. The velocities and accelerations are very noisy if we compute them directly from the joint angles using differentiations. Filtering of the joint angles data should be done because the arm movements is not expected to be high speed in the context of rehabilitation, and therefore any signals with frequency higher than $3 \mathrm{~Hz}$ are probably not due to the movement itself.

\subsubsection{Linear Kalman filter}

A linear Kalman filter is designed to estimate the velocities and accelerations from the joint angles. The state variables, $x$, are designated as

$$
x=\left[\begin{array}{c}
\text { angle } \\
\text { velocity } \\
\text { acceleration }
\end{array}\right]=\left[\begin{array}{l}
x_{k} \\
v_{k} \\
a_{k}
\end{array}\right]
$$

The system model for the filter is

$$
\begin{gathered}
x_{k+1}=A x_{k}+w_{k} \\
z_{k}=H x_{k}+v_{k}
\end{gathered}
$$

where $w_{k}$ and $v_{k}$ are the state transition noise and measurement noise respectively. The state transition matrix $A$ is

$$
A=\left[\begin{array}{ccc}
1 & \Delta t & 0.5 \Delta t^{2} \\
0 & 1 & \Delta t \\
0 & 0 & 1
\end{array}\right]
$$

and the state-to-measurement matrix $H$ is

$$
H=\left[\begin{array}{lll}
1 & 0 & 0
\end{array}\right]
$$


The system noise covariance, $Q$, and the measurement noise covariance, $R$, are designated as

$$
\begin{gathered}
Q=\left[\begin{array}{ccc}
N_{Q} & 0 & 0 \\
0 & N_{Q} / \Delta t & 0 \\
0 & 0 & N_{Q} / \Delta t^{2}
\end{array}\right] \\
R^{i}=N_{R}^{i}
\end{gathered}
$$

where $i=1,2, \ldots, 11$, denotes the joint numbers. The values of $N_{Q}$ and $N_{R}^{i}$ are approximated using the data given in [108], and are listed in Table 9.

Table 9: The values of $N_{Q}$ and $N^{i}{ }_{R}$ (approximated from data in [108])

\begin{tabular}{|c|c|}
\hline Noise Covariance & Estimated values \\
\hline$N_{Q}$ & $1.2^{\circ}=0.0209 \mathrm{rad}$ \\
\hline$N_{R}^{i}$ for $i=1,2,7,8,9,10,11$ & $1.2^{\circ}=0.0209 \mathrm{rad}$ \\
\hline$N^{3} R$ & $1.5^{\circ}=0.0262 \mathrm{rad}$ \\
\hline$N^{4}{ }_{R}$ & $8.0^{\circ}=0.1396 \mathrm{rad}$ \\
\hline$N^{5}{ }_{R}$ & $4.7^{\circ}=0.0820 \mathrm{rad}$ \\
\hline$N^{6}$ & $3.2^{\circ}=0.0559 \mathrm{rad}$ \\
\hline
\end{tabular}

\subsubsection{Calculation of joint torques}

The equations of motion of the arm model can be concisely written as

$$
T(q, \dot{q})+R(q, \dot{q})=M(q) \ddot{q}+C(q, \dot{q}) \dot{q}+G(q)+J(q)^{T} F
$$

where $M$ is the joint-space inertia matrix, $C$ is the Coriolis and centripetal coupling matrix, $G$ is the gravity loading, $T$ is the active muscular joint torques, $R$ is the passive joint resistance, and $F$ is the external forces transformed by an appropriate Jacobian $J$. The equations can be solved by using Lagrangian function, where the matrices are formed separately. It is very tedious for a model of 11 joints to be solved in this way, although it provides meaningful insights into each term of Eq. (3.61). A more efficient way to obtain the effective joint torques, that is, the left-hand side of Eq. (3.61), is using the recursive Newton-Euler formulation [124]. This is implemented using the function rne (recursive Newton-Euler method) in the Robotic Toolbox in Matlab. 
There are, however, some limitations on the solutions of the arm model. First, it was intended from the start that the sensors for the model would exclude force transducers. This is because it is very difficult, if possible, to measure contact forces from the environment during functional tasks. The weight of the transducers may also add on unnecessary load to the arm and the placement may obstruct the movement. Therefore, the arm model can only simulate free-moving arm motions and functional tasks that do not involve significant external forces, like combing of hair or zipping a coat, or only involve forces that can be modelled as a point mass, like drinking from a bottle or transferring objects.

Secondly, the joint torques, $Q(q, \dot{q})$, computed from the model are the effective joint torques, which are the vector sum of the active muscular torques, $T(q, \dot{q})$, and the passive joint resistance, $R(q, \dot{q})$. That is,

$$
Q(q, \dot{q})=T(q, \dot{q})+R(q, \dot{q})
$$

The model cannot differentiate between active and passive torques. For the computation of the muscle strengths based on the effective joint torques of active motions, the model of the muscle mechanics has to include a passive component. For assisted motions, like in the case of a Modified Ashworth Scale (MAS) examination, the active torques of the arm of the subject can be neglected. The mechanics behind active and passive torques are different and this shall be taken into consideration when using the effective torques to calculate muscle strengths.

\subsubsection{Validation and experimental results}

Validation of the arm model using only joint torques is challenging because it is very difficult to measure the joint torque directly [18], and unlike in lower limb studies, where walking and running are the standard tasks for validation, there is no standard set of arm motions in upper limb studies [125], thus making comparison with previous works difficult. Many studies in the literature validate the muscle forces or activations predicted by their arm models qualitatively using surface EMG signals [18], but this can only be done with a complete musculoskeletal model. At the present stage, we compare the maximum joint 
torques during the raising of arm in the frontal and sagittal planes with the published results of a model based on finite element method (FEM) by Van der Helm in [104].

\subsubsection{Experiment setup}

Four healthy male subjects (mean age 26.5 (SD 2.65) years, mean height 176.0 (SD 5.7) cm, and mean weight 61.5 (SD 3.9) kg) volunteered for the experiment after informed consent was obtained from them. The subjects stood upright with their arms resting naturally by the sides. Then they performed the following two motions: (a) raising the arm in the frontal plane, i.e., sideward, from the rest position to $90^{\circ}$ elevation angle, and then over the head at about $180^{\circ}$ elevation angle; (b) same as (a), but in the sagittal plane, i.e., frontward.

\subsubsection{Results and discussions}

Figure 19a and 19b show the profiles of the torques at the dominant DOFs during the experiments. The maximum joint torque of all four subjects occurred when the arm was holding its position at $90^{\circ}$ elevation angle, shown in the plots in Figure 19 from $30-70 \%$ motion cycle. This is because the static moments caused by the weights of the arm segments at the shoulder joints are largest at this position. It also shows that total joint torque is dominated by the static component (the gravity loading, $G(q)$ ) instead of the dynamic components (the inertial term, $M(q) \ddot{q}$, and the Coriolis term, $C(q, \dot{q}) \dot{q})$. In the context of stroke rehabilitation, the motions of the arm are relatively slow, the gravity loading, $G(q)$, in the equation of motion becomes the dominant term [124]. Since $G\left(q_{i}\right)=$

$-\sum_{j=1}^{n} m_{j} g^{T} \frac{\partial c_{j}}{\partial q_{i}}$, where $n$ is the number of joints, $g$ is the gravity vector, $m_{j}$ and $c_{j}$ are the mass and center of mass of link $j$, we see that the joint torque is dependent on the link mass. The link mass is in turn computed from the body mass of the subject. So there is a need to scale the torque by the body mass when performing comparisons. The maximum joint torques of the dominant DOFs 
during the experiments in the sagittal and frontal planes are shown in Table 10 and 11 respectively.
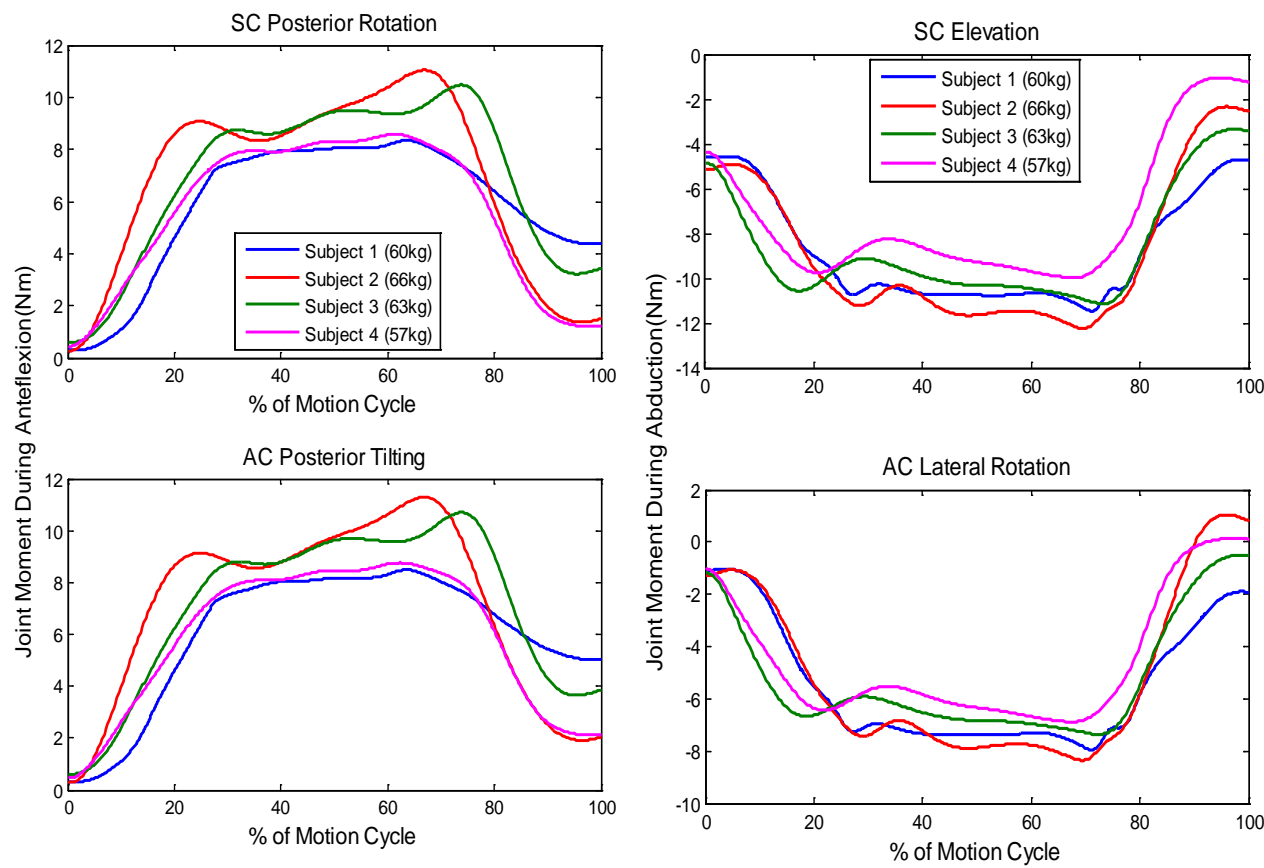

Figure 19: Profiles of torque at dominant DOFs during arm raising in: (a) Sagittal plane (DOFs: SC posterior rotation and AC posterior tilting); (b) Frontal plane (DOFs: SC elevation and AC lateral rotation) 
Table 10: Maximum Joint Torques during Arm Anteflexion (Sagittal Plane)

\begin{tabular}{|c|c|c|c|c|c|c|}
\hline \multirow{3}{*}{ Subj } & \multicolumn{2}{|c|}{$\begin{array}{c}\text { SC Posterior } \\
\text { Rotation }\end{array}$} & \multicolumn{2}{c|}{ AC Posterior Tilting } & \multicolumn{2}{c|}{$\begin{array}{c}\text { GH Sagittal } \\
\text { Elevation }\end{array}$} \\
\cline { 2 - 7 } & $\begin{array}{c}\text { Max } \\
\text { Torque } \\
(\mathrm{Nm})\end{array}$ & $\begin{array}{c}\text { Scaled } \\
\text { Torque } \\
(\mathrm{Nm} / \mathrm{kg})\end{array}$ & $\begin{array}{c}\text { Max } \\
\text { Torque } \\
(\mathrm{Nm})\end{array}$ & $\begin{array}{c}\text { Scaled } \\
\text { Torque } \\
(\mathrm{Nm} / \mathrm{kg})\end{array}$ & $\begin{array}{c}\text { Max } \\
\text { Torque } \\
(\mathrm{Nm})\end{array}$ & $\begin{array}{c}\text { Scaled } \\
\text { Torque } \\
(\mathrm{Nm} / \mathrm{kg})\end{array}$ \\
\hline 1 & 8.44 & 0.141 & 8.60 & 0.143 & 6.34 & 0.106 \\
\hline 2 & 11.04 & 0.167 & 11.29 & 0.171 & 8.02 & 0.122 \\
\hline 3 & 10.47 & 0.166 & 10.70 & 0.170 & 7.81 & 0.124 \\
\hline 4 & 8.57 & 0.150 & 8.76 & 0.154 & 6.63 & 0.116 \\
\hline $\begin{array}{c}\text { Mean } \\
\text { (SD) }\end{array}$ & & $\begin{array}{c}\mathbf{0 . 1 5 6} \\
\mathbf{( 0 . 0 1 3 )}\end{array}$ & & $\mathbf{0 . 1 6 0}$ & & $\begin{array}{c}\mathbf{0 . 1 1 7} \\
\mathbf{( 0 . 0 0 8})\end{array}$ \\
\hline $\begin{array}{c}\text { Helm } \\
{[104]}\end{array}$ & 11.50 & $\mathbf{0 . 1 4 9}$ & 11.90 & $\mathbf{0 . 1 5 5}$ & 9.30 & $\mathbf{0 . 1 2 1}$ \\
\hline \%Diff & & $\mathbf{4 . 4 9}$ & & $\mathbf{3 . 1 3}$ & & $\mathbf{- 3 . 4 2}$ \\
\hline
\end{tabular}

Table 11: Maximum Joint Torques during Arm Abduction (Frontal Plane)

\begin{tabular}{|c|c|c|c|c|c|c|}
\hline \multirow{2}{*}{ Subj } & \multicolumn{2}{|c|}{ SC Elevation } & \multicolumn{2}{c|}{ AC Lateral Rotation } & \multicolumn{2}{c|}{$\begin{array}{c}\text { GH Frontal } \\
\text { Elevation }\end{array}$} \\
\cline { 2 - 7 } & $\begin{array}{c}\text { Max } \\
\text { Torque } \\
(\mathrm{Nm})\end{array}$ & $\begin{array}{c}\text { Scaled } \\
\text { Torque } \\
(\mathrm{Nm} / \mathrm{kg})\end{array}$ & $\begin{array}{c}\text { Max } \\
\text { Torque } \\
(\mathrm{Nm})\end{array}$ & $\begin{array}{c}\text { Scaled } \\
\text { Torque } \\
(\mathrm{Nm} / \mathrm{kg})\end{array}$ & $\begin{array}{c}\text { Max } \\
\text { Torque } \\
(\mathrm{Nm})\end{array}$ & $\begin{array}{c}\text { Scaled } \\
\text { Torque } \\
(\mathrm{Nm} / \mathrm{kg})\end{array}$ \\
\hline 1 & 11.50 & 0.192 & 9.33 & 0.156 & 7.94 & 0.132 \\
\hline 2 & 12.25 & 0.186 & 9.85 & 0.149 & 7.66 & 0.116 \\
\hline 3 & 11.14 & 0.177 & 10.48 & 0.166 & 6.95 & 0.110 \\
\hline 4 & 9.98 & 0.175 & 9.29 & 0.163 & 6.90 & 0.121 \\
\hline $\begin{array}{c}\text { Mean } \\
\text { (SD) }\end{array}$ & & $\begin{array}{c}\mathbf{0 . 1 8 3} \\
\mathbf{( 0 . 0 0 8})\end{array}$ & & $\mathbf{0 . 1 5 9}$ & & $\mathbf{0 . 1 2 0}$ \\
\hline $\begin{array}{c}\text { Helm } \\
{[104]}\end{array}$ & 14.80 & $\mathbf{0 . 1 9 2}$ & 13.10 & $\mathbf{0 . 1 7 0}$ & 10.20 & $\mathbf{0 . 1 3 3}$ \\
\hline \%Diff & & $\mathbf{- 4 . 9 2}$ & & $\mathbf{- 6 . 9 2}$ & & $\mathbf{- 1 0 . 8 3}$ \\
\hline
\end{tabular}

The standard deviations of the scaled torques among the subjects are small (relative standard deviation $<9 \%$ ), which means that there is consistency in the sensor and model interface. The means of the scaled torque are compared with data from Helm [104], and the differences between the two models are small $(<11 \%)$. The inertial parameters in Helm [104] are obtained using detailed scan of a single cadaver, and there is no validation on the results. The comparison between the two models should not be taken as a conclusive validation on the 
accuracy of our model. However, the small discrepancy in the scaled maximum torques shows that our model is able to have an estimation of the joint torques with accuracy comparable to a model that based on parameters that are not possible to measure in a clinical setting. This small discrepancy may be due to the fact that the maximum joint torques in Helm [104] are computed from static analysis of a FEM model whereas our model used ambulatory data from sensors. 


\section{A Musculoskeletal Model for the Human Upper Limb}

\subsection{Introduction}

Computational models of musculoskeletal properties of the human arm are developed to provide estimations for the muscle forces because these forces cannot be directly measured non-invasively. Many researchers have derived a variety of techniques in the modelling; each caters for different purposes and applications. However, the steps for the modelling process are largely similar. They are: (1) define the origin and insertion sites of the muscles relative to the bone structures; (2) define the muscle path relative to joint motion; (3) define the muscle contraction dynamics model; (4) compute the moment arms of the muscles; (5) set up optimization routine to solve muscle redundancy problem [18].

\subsection{Defining origins and insertions of the muscles}

The muscle system model for the upper limb consists of 22 muscles (see Appendix A). Some of the muscles that span the shoulder are fan-shaped and their attachment sites are over a large area (e.g. trapezius and pectoralis major). Some muscles have multiple attachment points (e.g. triceps brachii and biceps brachii). For these muscles, they are divided into two or more bundles, with each bundle having its own line of actions. It is a difficult and expensive process to obtain the exact position of each attachment site of all the muscles of individual subject, which is not feasible as a routine procedure in a clinical setting. Some researchers make use of scanned data of cadavers to define the attachment sites of the muscles as input parameters for their models [21, 70, 75]. The coordinates of the origins and insertions of the muscles in our model are based on the data listed in [70], adjusted with respect to the local frames of the bones (see Appendix 
A). Although the data is obtained from a single cadaver, we can use it as a basis in the development of the arm model.

\subsection{Defining muscle paths}

The muscle path describes the way a muscle is connected from the origin to the insertion relative to the posture of the body. It is an important aspect in muscle modelling as it determines the change in the muscle length during movements of the body, which in turns affects the force generated by the muscle. There are two main approaches that are used to model muscle path: the straight-line model and the centroid-line model. The straight-lined model uses a straight line to connect the centroids of the muscle attachment areas. This method is easy to implement but it ignores the complexity of muscle wrapping around tendon sheath and other muscles. The centroid-line approach attempts to address this problem by using a line that passes through the locus of the cross-sectional centroids of the muscle [126]. The problem with this model lies in the difficulty to obtain the locations of the cross-section centroid which are dependent on the configuration of the joints. In [69], Garner and Pandy introduce an alternate approach, called the obstacle-set method, that can define the muscle path for all joint configurations. It is based on the assumption that anatomical structures like tendons and other muscles can be represented by regular-shaped, rigid bodies, called obstacles, and the path of the muscle that wraps around these obstacles can move freely over them. There are four types of obstacle defined by the method: single sphere, single cylinder, double cylinder and sphere-capped cylinder (see Figure 20, from [69]). 

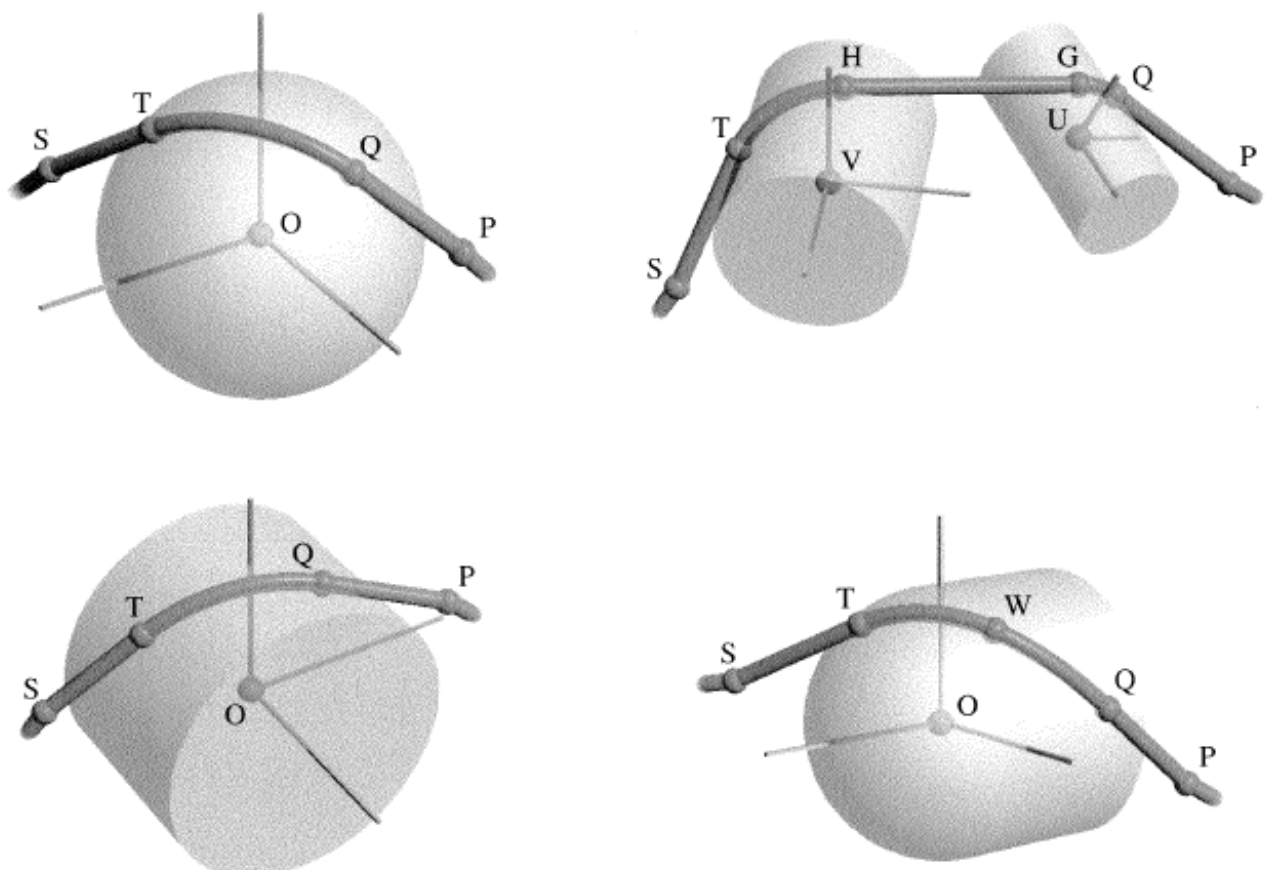

Figure 20: The regular-shaped rigid bodies used as obstacles in obstacle-set method: single sphere, single cylinder, double cylinder and sphere-capped cylinder.

The muscle path is described by the lines joining points that represent origin, insertion, via points and points on the obstacle surface. The origin, insertion, via points and the obstacles are attached to the various local frames of the bones, so when the bones move relative to each other, the positions of the various points can be computed. The obstacles and their local frames for each of the 22 muscles are listed in Appendix A.

\subsection{Muscle contraction dynamics model}

Besides muscle path, the magnitude of the muscle force also depends on its activation level and its force-generation properties defined by force-length and force-velocity relationships. The most commonly used muscle contraction dynamics model is the Hill-type muscle model [127]. A Hill-type muscle model consists of a contractile element (CE), and two non-linear spring elements, one in series (SE) and the other in parallel (PE) (see Figure 21). 


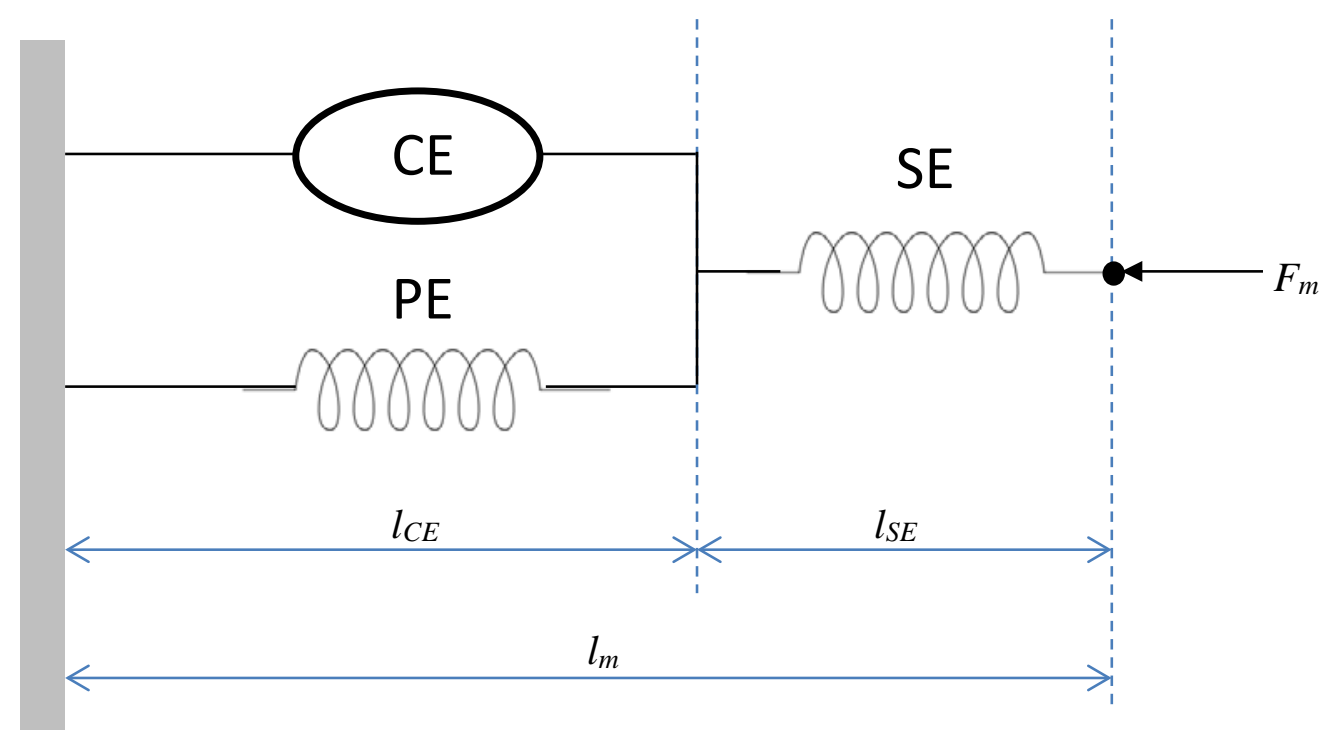

Figure 21: Three-element Hill type muscle model consists of a contractile element (CE) and two non-linear spring elements, parallel element (PE) and series element (SE). $l_{C E}$ is the length of $C E$ and $l_{S E}$ is the length of SE, both add up to the length of the muscle element, $l_{m} . F_{m}$ is the muscle force.

The muscle force produced by the muscle, $F_{m}$, is defined as $[128,129]$

$$
F_{m}=F_{C E}+F_{P E}
$$

where $F_{C E}$ and $F_{P E}$ are the forces at the $C E$ and PE respectively.

The force produced by the contractile element, $F_{C E}$ is

$$
F_{C E}=F_{\max }^{i s o} f_{l} f_{v} A
$$

where $F_{\text {max }}^{i s o}$ is the maximum isometric muscle force; $A$ is the activation level, a value ranging from 0 (no activation) to 1 (maximum activation); $f_{l}$ and $f_{v}$ are the normalized force-length relationship and force-velocity relationship respectively. The normalized force-length relationship is a bell-shaped curve defined as:

$$
f_{l}\left(l_{C E}\right)=e^{-\frac{\left|l_{C E}-l_{o p t}\right|^{2}}{w^{2}}}
$$

where $l_{\text {opt }}$ is the optimal muscle fiber length, and $w$ is a parameter that is usually set to be $0.5 l_{\text {opt }}$. The normalized force-velocity relationship is defined by two piecewise continuous curves: 


$$
f_{v}\left(v_{C E}\right)=\begin{gathered}
\frac{v_{\max }-v_{C E}}{v_{\max }+k v_{C E}}\left(v_{C E} \leq 0\right) \\
N+(N-1) \frac{v_{\max }+v_{C E}}{7.56 k v_{C E}-v_{\max }}\left(v_{C E}>0\right)
\end{gathered}
$$

where $v_{\max }$ is a parameter that defines the maximum rate of change of the muscle length when $f_{v}$ is zero; $k$ is the shape factor of the curves; and $N$ is a parameter termed as eccentric force enhancement.

The resistive force in the parallel element only starts to have effect when the $l_{C E}$ is longer than $l_{o p t}$, and the force-length relationship is parabolic:

$$
F_{P E}\left(l_{C E}\right)=F_{\max }^{i s o} \frac{\left(l_{C E}-l_{o p t}\right)^{2}}{\varepsilon^{2}}\left(l_{C E}>l_{o p t}\right)
$$

where $\varepsilon$ is a normalization parameter.

The muscle force in $\mathrm{CE}$ and $\mathrm{PE}$ is equivalent to the force in SE as they are in series. Therefore the muscle force is also defined as:

$$
F_{m}=F_{S E}\left(l_{S E}\right)=F_{\max }^{i s o} \frac{\left(l_{S E}-l_{\text {slack }}\right)^{2}}{\varepsilon_{\text {ref }}{ }^{2}}\left(l_{S E}>l_{\text {slack }}\right)
$$

where $l_{\text {slack }}$ is the tendon slack length; and $\varepsilon_{r e f}$ is a normalization parameter.

\subsection{Moment arm of a muscle force}

The tendency of a muscle to rotate a bone about a joint is described by the muscle’s moment arm. In the simplest case, a straight-line muscle whose origin and insertion points connect two adjacent bodies connected by a pin joint, the moment arm can be viewed as the same as conventional moment arm in mechanical engineering, where its definition is "the distance from the muscle's line of action to the joint's center of rotation”. However, in reality, most muscles may either span several joints, or follow a contorted crossing over other body parts, or both. For these situations, the definition of moment arm becomes more complex.

There are two methods commonly used to measure or estimate the moment arm of a muscle: (a) the geometric method, and (b) the tendon excursion method [130]. In the geometric method, the center of joint rotation and the muscle line of action are estimated using X-rays, CT scans or MRI scans, and the moment arm is the 
perpendicular distance between the two. The usage of these machines limits the usefulness of this method in clinical applications. In the tendon excursion method, the change in length of the muscle-tendon unit is measured or estimated as a function of the joint angle, and the instantaneous moment arm is defined as the slope of the curve when muscle length is plotted against the joint angle. The tendon excursion has the advantage of not requiring the knowledge of joint center of rotation and muscle line of action, but the quantities derived from this method is difficult to interpret geometrically. And for a muscle that spans more than a joint, the angular motion of the joint of interest has to be measured while other joints are held rigid. This proves to be a very difficult task especially for upper limb where certain degrees of freedom of the joints cannot be moved independently.

There are a few studies on the measurement of the moment arms of the upper arm muscles relative to the shoulder [131-133] and elbow joints [134] in the literature. There are large discrepancies in the estimated moment arms in some muscles. For example, deltoid's moment arm for shoulder abduction ranged from about $4 \mathrm{~cm}$ to $1 \mathrm{~cm}$ [135]. This large variation is due partly to the differences in the modelling methods used in each study. The differences include the degree of freedom and the constraints of the model, and the definition of the origin and insertion of the muscles. This shows that there are still some difficulties to overcome before we can arrive at a standardized method to compute the moment arms for the muscles of the upper limb.

In [135], Holzbaur et al. provide a list of muscle modelling parameters that includes the average moment arms for upper limb muscles. In our model we used the average moment arms in this list as the base values for an optimization procedure to determine the moment arms for our model. The procedures are as follow:

1. Formulate the optimization problem for the muscle sharing problem (which will be discussed in the next section), but include the moment arm of each muscle as one of the optimizing variables. The upper bounds and 
lower bounds of the moment arm of each muscle are based on the maximum and minimum values we can find in the literature [131-135]. For those muscles where no data is available, we assume a $\pm 20 \%$ of the absolute value of the moment arm as the bounds.

2. The subject performs a set of arm motions: (a) raising the arm in the sagittal plane; (b) raising the arm in the frontal plane; and (c) drinkingfrom-a-cup motion. These motions invoke all the degree of freedom at the shoulder and elbow joints. The data captured by the sensors are used in the optimization procedure.

3. After the optimization routine, the moment arms of the muscles are collated and a lookup table is set up where the moment arms of each muscle are tabulated against the joint angles. This lookup table will be used for the subject for subsequent applications of the model.

The procedure ensures that there is consistency in the moment arm of the muscles for a particular subject using the model. However, the data from [135] was based on a single cadaver specimen. To make the model viable for different subject, the average moment arms have to be scaled using the height and lengths of clavicle, humerus and forearm.

\subsection{Muscle force sharing problem}

The human musculoskeletal system is characterized by having redundancy in muscular actuation at joints, that is, the number of muscle responsible for a motion is larger than the number of degree of freedom. In the context of our arm model, for example, the abduction of the arm is actuated by deltoid, pectoralis major, latissimus dorsi, and teres major. Such redundancy of muscular load sharing problem is usually solved by optimization methods [18], where the most established and common one is inverse dynamics-based static optimization. The joint torques we have computed previously in stage 1 are used as the input for the constraints for the optimization routine. The muscle force sharing problem is formulated as: 
Minimize

$$
J(\vec{A})=\sum_{i=1}^{n}\left(\frac{F_{m}^{i}\left(A_{i}\right)}{\operatorname{PCSA}_{i}}\right)^{2}
$$

Subject to:

$$
\begin{gathered}
\boldsymbol{R} \vec{F}_{m}(\vec{A})-\vec{M}_{j}=\overrightarrow{0} \\
0 \leq A_{i} \leq 1
\end{gathered}
$$

The objective of the problem, $J(\vec{A})$, is to minimize sum of muscle stress squared. This is done by finding the optimal activation levels of each muscle, $\vec{A} \in \mathbb{R}^{n}$, where $n$ is the number of muscles in the model, and the activation level is between 0 (no activation) to 1 (maximum activation). PCSA is the physiological crosssectional area of individual muscles. PCSA is typically used to describe the contraction properties of muscles, and the muscle stress is defined as the muscle force per unit PCSA. The optimization is subjected to the constraint that the moments at the joints, $\vec{M}_{j} \in \mathbb{R}^{N}$, where $N$ is the number of joints in the model, equal to the product of moment arm and the muscle force. $\boldsymbol{R} \in \mathbb{R}^{N \times n}$ is the moment arm matrix for the model.

The choice of objective function for muscle force sharing problem depends on the body parts and the objective of the research. In a biomechanical context, the objective function is also known as the performance criterion. It is a measure of performance of the muscles based on the assumption that the muscular system works in a way that minimizes the activation levels. For the research on upper limb, minimizing sum of muscle stress squared is the most commonly used objective function [18]. The advantage of using muscle stress over using muscle forces in the objective function is that it prevents the overuse of favorably located muscles. Computationally, it is also very efficient and stable, a crucial criterion for the model to be useful clinically. 


\subsection{Results and discussions}

There is no simple and universal method to validate the muscle forces predicted by models. This is mainly because measuring the muscle forces directly is only possible in highly controlled and invasive situations that involves only one or two degrees of freedom $[16,136]$. From the literature, most of the model predictions compare muscle loading or activation patterns against EMG data as an estimate of validity [18]. Such comparisons only provide qualitative validation but cannot verify the magnitude of the calculated muscle forces.

To validate the muscle force estimations of our model, we carry out a simple experiment that involved a subject performing two sets of motion: (a) lifting a $5 \mathrm{~kg}$ deadweight three times at a standing position; and (b) reaching out to a bottle of water and drink from it. Besides the IMUs attached to the arm (see Section 3.1.2), four EMG electrodes (Trigno ${ }^{\mathrm{TM}}$ Wireless Systems and Smart Sensors, Delsys Inc.) were attached to the deltoid muscle, the biceps brachii, the triceps brachii, and the brachioradialis (see Figure 22).

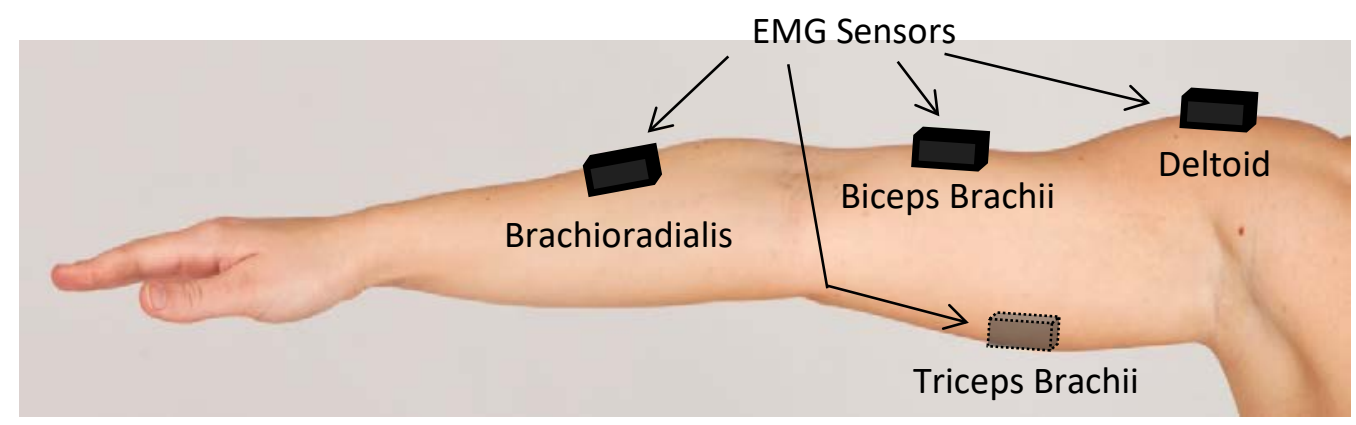

Figure 22: The placement of EMG sensors on the deltoid, the biceps brachii, the triceps brachii, and the brachioradialis.

The maximum voluntary contraction (MVC) of the biceps brachii of the subject was measured prior to the experiment. As the MVCs of the other muscles are difficult to measure accurately, they are estimated based on the MVC of the 
biceps and the relative PCSAs of the muscles. In the experiment, each motion was repeated six times and the muscle forces were computed as the activation level (\%MVC) of the particular muscle. The results are shown in Figure 23 and 24 for the (a) drinking and (b) weight-lifting motions respectively.
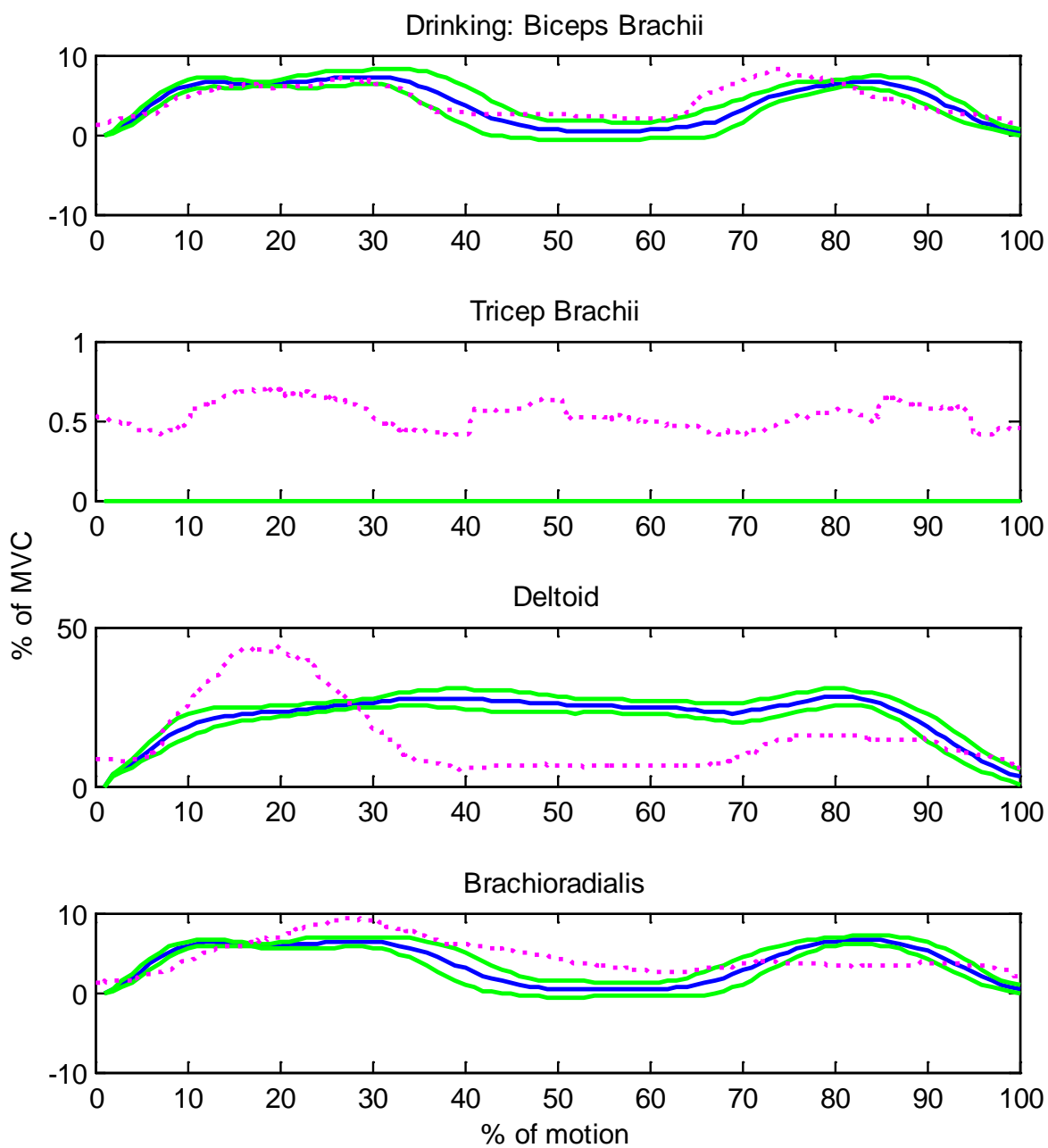

Figure 23: Muscle activation level (\%MVC) predicted by the model and EMG signals of four arm muscles during drinking motion. The blue line is the average muscle force of six trials and the green lines are the standard deviation of the trials. The dotted line is the corresponding EMG signal. 

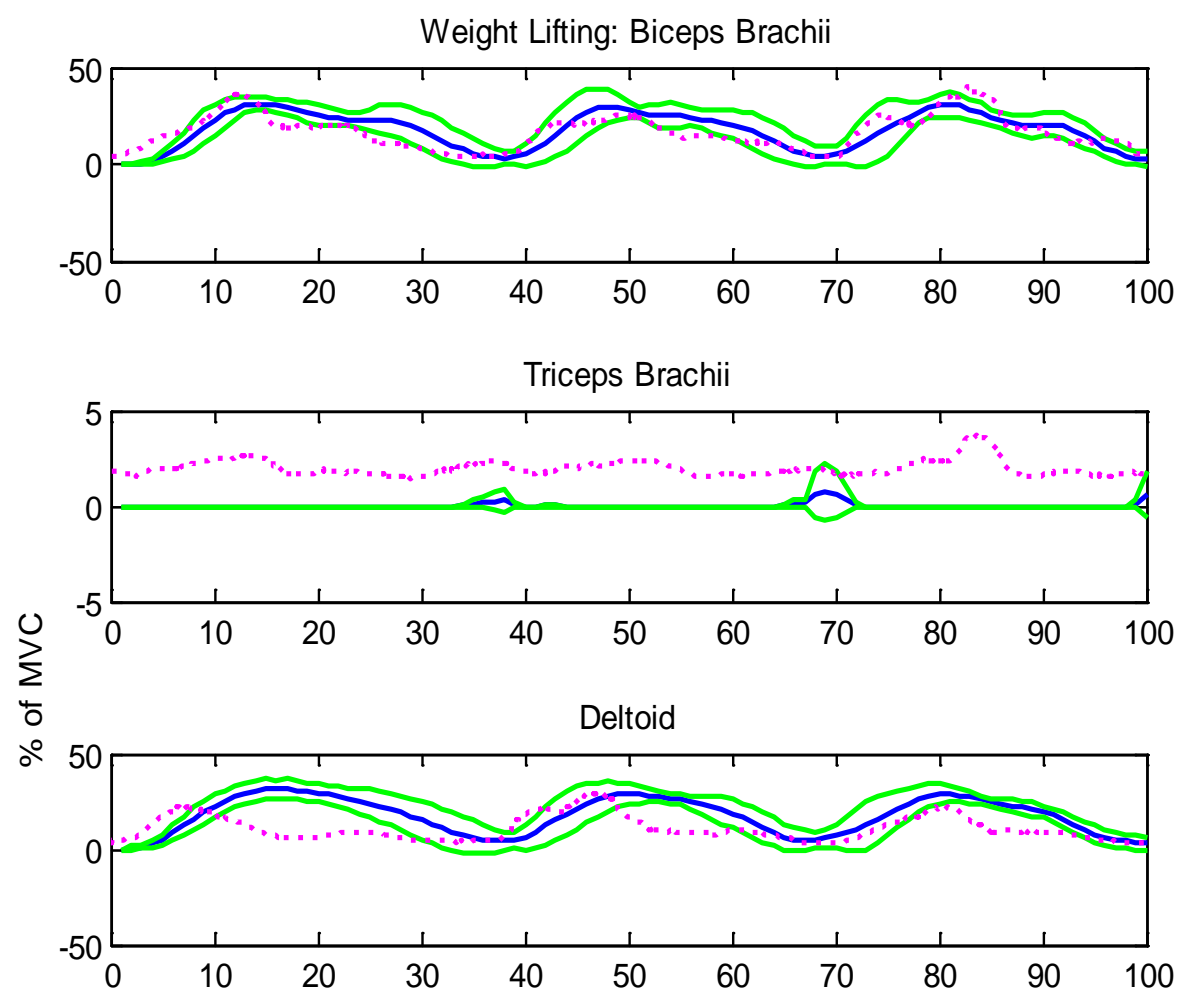

Brachioradialis

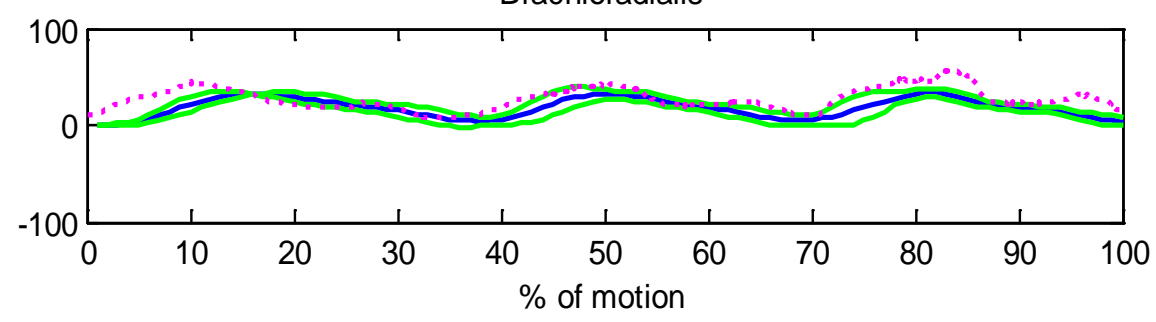

Figure 24: Muscle activation level (\%MVC) predicted by the model and EMG signals of four arm muscles during weight-lifting motion. The blue line is the average muscle force of six trials and the green lines are the standard deviation of the trials. The dotted line is the corresponding EMG signal. 
To establish the comparison between the estimated muscle forces from the model and the EMG signals, we can compute the cross-correlation coefficient [137], $r_{A E}$, defined as [138]:

$$
r_{A E}=\frac{c_{A E}}{\sqrt{c_{A A C E E}}}
$$

where

$$
\begin{gathered}
c_{A E}=\sum_{i=1}^{100}\left[\left(A_{i}-\bar{A}\right)\left(E_{i}-\bar{E}\right)\right] \\
c_{A A}=\sum_{i=1}^{100}\left(A_{i}-\bar{A}\right)^{2} \\
c_{E E}=\sum_{i=1}^{100}\left(E_{i}-\bar{E}\right)^{2}
\end{gathered}
$$

and $A_{i}$ is the estimated activation level at $i \%$ of the motion, $\bar{A}$ is the mean of the activation level; $E_{i}$ is the EMG signal level at $i \%$ of the motion, and $\bar{E}$ is the mean of the EMG signal level. Both signals are measured in \%MVC. The crosscorrelation coefficients of each muscle for the two sets of motion are tabulated in Table 12.

Table 12: Cross-correlation coefficients, $r_{A E}$, between estimated muscle activation level and EMG signal level of each muscle for the weight-lifting and drinking motions.

\begin{tabular}{|c|c|c|c|c|c|c|c|}
\hline \multicolumn{4}{|c|}{ Weight-lifting } & \multicolumn{4}{c|}{ Drinking } \\
\hline $\begin{array}{c}\text { Bi- } \\
\text { ceps }\end{array}$ & $\begin{array}{c}\text { Tri- } \\
\text { ceps }\end{array}$ & $\begin{array}{c}\text { Del- } \\
\text { toid }\end{array}$ & $\begin{array}{c}\text { Brachio- } \\
\text { radialis }\end{array}$ & $\begin{array}{c}\text { Bi- } \\
\text { ceps }\end{array}$ & $\begin{array}{c}\text { Tri- } \\
\text { ceps }\end{array}$ & $\begin{array}{c}\text { Del- } \\
\text { toid }\end{array}$ & $\begin{array}{c}\text { Brachio- } \\
\text { radialis }\end{array}$ \\
\hline 0.792 & 0.047 & 0.677 & 0.710 & 0.818 & 0.159 & 0.225 & 0.5959 \\
\hline
\end{tabular}

The four muscles are selected for the validation experiment mainly because they are superficial and large, such that the EMG signals are stronger and less ambiguous. From Figure 23 and 24, we see that the estimated activation levels of biceps brachii are very similar to the EMG signals. This is confirmed by the cross-correlation coefficients in Table 12, where both sets of motion registered a high correlation of about 0.8 . The cross-correlation coefficients are also relatively high for brachioradialis in both motions. In contrast, it is apparent from the figures and table that the estimated activation levels of triceps brachii and deltoid do not correlate very well with the EMG signals, especially for the drinking 
motion. Both motions utilize a significant amount of activations on biceps brachii and brachioradialis, coupling with the fact that both muscles are easily palpable and the sensors can be placed on the muscle precisely, the EMG signals can be compared with the computed muscle activations more accurately. On the other hand, these motions do not make use of the triceps brachii significantly. Referring to Figure 23 and 24, the EMG signal levels of triceps brachii for both motions are less than 5\%MVC, whereas the model predicted activation levels are close to zero. The low EMG signal levels might be the results of crosstalk from other nearby muscles. This shows that these two motions are not suitable for the validation of the triceps muscle. For the deltoid muscle, it is modelled as three separate bundles: the clavicular, the acromial, and the scapular. There is some overlapping among the three bundles and EMG signal might come from all three bundles. So to compute the predicted muscle activation, the sum of the activations of all three bundles is taken to be the overall activation. However, from Figure 23 and 24, we see that the EMG signal contribution from each bundle changes during the course of the motion. Since there is no way to determine the contribution from each bundle, deltoid cannot be validated using the EMG signals.

The high correlations between the EMG signals and the predicted muscle activations for biceps and brachioradialis provide confidence in the validity of the model. As an alternative way, we propose using the results of the applications of the model to indirectly validate the model's predictions, and thus establishing the usefulness of the model. The application in spasticity assessment is the subject of the next chapter. 


\section{Application: Assessment of Spasticity}

In the Stage 3 of the project, the musculoskeletal model of the human upper limb is applied in the application to quantify the tonic stretch reflex threshold (TSRT) of stroke patient subject so as to assess the severity of spasticity. This is to complement the current practice of using Modified Ashworth Scale (MAS) for the assessment by providing an alternative with quantified measurement. The following sections present the methods used, describe the experimental procedures and discuss the implications of the results.

\subsection{Introduction}

Spasticity is the most common and serious complications resulted from stroke and spinal cord lesion. It may be defined as motor disorder characterized by a velocitydependent exaggeration of stretch reflexes [11]. Quantification of the severity of spasticity can increase the efficacy of treatment and rehabilitation. However, clinicians generally agree that it is easy to recognize spasticity but very difficult to quantify it. The involuntary resistance to stretch maybe a result of changes in reflex threshold or reflex gain, or a combination of both [12], but the actual mechanism is still unclear [139]. One major difficulty to perform a quantitative assessment of spasticity is that some other diseases like Parkinson show similar symptoms in muscle resistance to passive motions [140]. The current common method used to quantify spasticity clinically is making use of the Modified Ashworth Scale (MAS) [34]. It is a very simple-to-use test but it only provides qualitative and subjective results. The velocity-dependency of spasticity distinguishes it from other syndromes but this is very difficult to identify using MAS examination [14]. Another less common method in quantifying spasticity is the pendulum test $[141,142]$. Potentially, the pendulum test provides a more objective and reliable than MAS, but it requires equipment like 
electrogoniometers [143], two-dimensional video analysis [144], and magnetic tracking system [145], and the clinicians have to be trained to handle these complex systems. This is a possible reason why pendulum test is not routinely used by most clinicians [146].

Some researchers have proposed to use the Tonic Stretch Reflex Threshold (TSRT) as the parameter to quantify spasticity $[12,14]$. TSRT is a measure of the joint angles when stretch reflex occurs at different velocities. It is an attractive alternative to MAS because it directly captures the velocity-dependency characteristic. The stretch reflexes in these studies are identified using Electromyography (EMG) sensors. However, besides the accuracy and reliability problems of the non-invasive EMG [45], there are many issues to resolve before TSRT measurement can become a routine protocol clinically. First, in these studies, only one or two major muscles' activities are measured. The complexity of spasticity cannot be fully captured. Secondly, the EMG sensors have to be used together with motion sensors, where the former identifies the muscle stretch reflexes and the latter measures the velocity. The costs and trainings needed to operate these instruments may be hindrances to promote the method.

We propose a method to address the issues faced by the current methods of measuring TSRT, using the musculoskeletal arm model we have developed. This section discusses the method and presents preliminary results. 


\subsection{Methods}

To discuss the method we are proposing we need to look at the definition of stretch reflex threshold (SRT). SRT represents the joint angle at which the involuntary recruitment of muscles begins. Researches have shown that the SRT of chronic stroke patients has significant differences from healthy people [147, 148], it is dependent on the joint angular velocity. To capture the velocity dependency characteristic of spasticity, we measure the dynamic stretch reflex threshold (DSRT). As the name implies, DSRT is the SRT at different joint angular velocities. Higher velocities invoke stretch responses at smaller joint angles. If we perform the stretches at different velocities, we can plot the DSRT against the joint angular velocities. Linear regression through the DSRTs is used to estimate the tonic stretch reflex threshold (TSRT) at zero velocity [12]. (See Figure 25.)

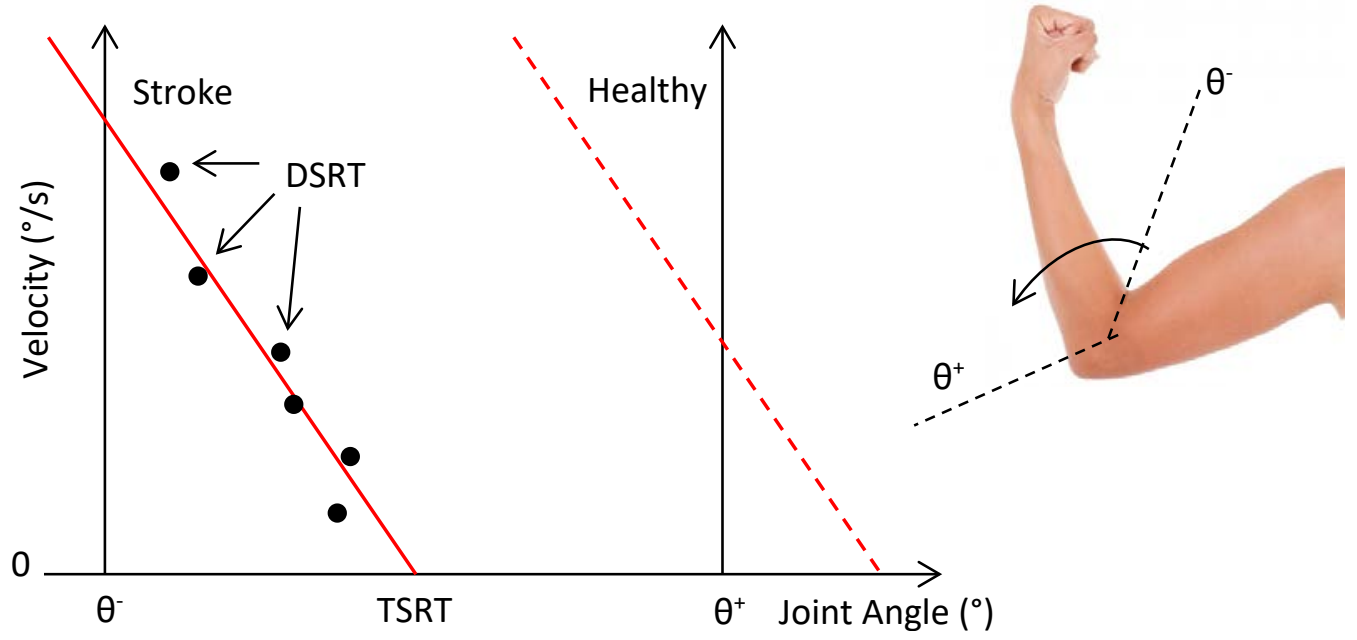

Figure 25: DSRTs are measured at different angular velocities and TSRT is estimated at the zero velocity via extrapolation of the linear regression. The maximum flexed position, $\theta^{-}$, and the maximum extended position, $\theta^{+}$, define the biomechanical range of the joint. For healthy subjects, the TSRT is beyond the biomechanical range. 
The method we propose here aims to solve the issues related to relying on EMG signal measurements to estimate the TSRT. The equations of motion for the arms are:

$$
\boldsymbol{\tau}-\boldsymbol{J}^{T} \boldsymbol{f}=\boldsymbol{M}(\boldsymbol{q}) \ddot{\boldsymbol{q}}+\boldsymbol{C}(\boldsymbol{q}, \dot{\boldsymbol{q}}) \dot{\boldsymbol{q}}+\boldsymbol{G}(\boldsymbol{q})
$$

where $\boldsymbol{\tau}$ is the active joint torques; $\boldsymbol{f}$ is the contact forces at the wrist and hand, transformed by the Jacobian, $\boldsymbol{J} ; \boldsymbol{M}, \boldsymbol{C}$ and $\boldsymbol{G}$ are the inertial, Coriolis, and gravitational terms respectively; and $\boldsymbol{q}, \dot{\boldsymbol{q}}, \ddot{\boldsymbol{q}}$ are the generalized joint coordinates, velocities and accelerations. In a typical session for assessment of spasticity of the upper limb using Modified Ashworth Scale (MAS), the therapist abducts the arm of the patient using one hand supporting at the elbow to about $90^{\circ}$ and places the forearm in a fully flexed position. Then the therapist extends the patient's forearm in a swift motion (see Figure 26).

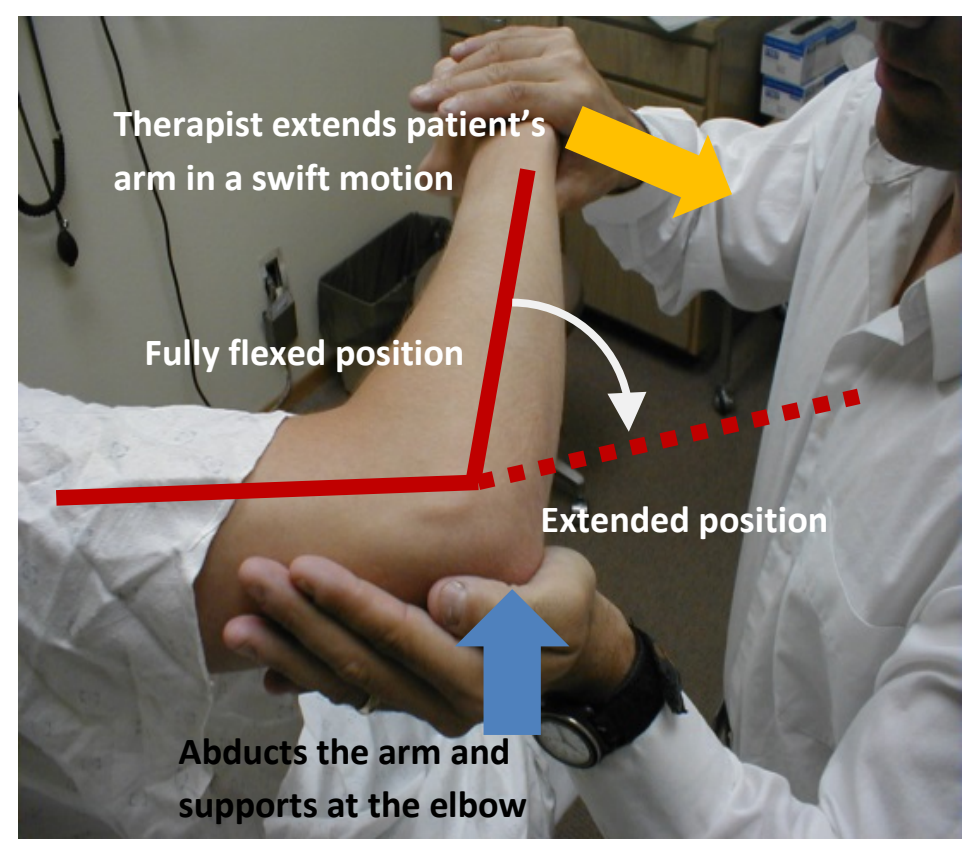

Figure 26: Elbow extension assessment for spasticity. The therapist supports the patient's elbow with one hand while extending the forearm with the other hand.

If we were to compute the muscle activations of the patient's arm, we need to measure the motions of both the therapist and the patient. This is because the 
unknowns in Eq. 5.1 are the active joint torques, and the contact forces between the therapist's hand and patient's wrist. One of the limitations of the arm model is the inability to handle dynamic contact forces (see Section 3.7.2). However, if we make the following three assumptions, we can eliminate the need to measure the contact forces in this situation.

1. The contact force experienced by the patient is equal and opposite to the force experienced by the therapist:

$$
\boldsymbol{f}_{p}=-\boldsymbol{f}_{t}
$$

where the subscripts $p$ and $t$ denote patient and therapist respectively. Both $\boldsymbol{f}_{p}$ and $\boldsymbol{f}_{t}$ are expressed in the inertial frame. As both arms of the subjects do not come in contact with other object, this is a reasonable assumption. The contact between the participant and the therapist is also assumed to be a point contact. It is a simplification from the actual area contact, as an area contact is too complex to be modelled in the method and be useful clinically. As far as measuring TSRT is concerned, this simplification would not cause too much deviation from the more realistic area contact as the contact is localized in a small area.

2. The time taken by the therapist to react to the sudden resistance is much longer than the sampling period of the sensors. That is, between two consecutive time steps up till the resistance set in, we assume that the change in the active joint torque of the therapist is very small:

$$
\boldsymbol{\tau}_{t}^{2} \approx \boldsymbol{\tau}_{t}^{1}
$$

where the superscripts 1 and 2 denote two consecutive time steps. As the reaction time of human muscle is about 200 to $300 \mathrm{~ms}$ [149], and the sampling period is $7.8 \mathrm{~ms}$, this assumption is reasonable too.

Using these two assumptions, we can combine the equations of motion of the patient and the therapist to compute the change in the joint torques of the patient between two consecutive time steps, $\Delta \boldsymbol{\tau}_{p}$ :

$$
\Delta \boldsymbol{\tau}_{p}=\boldsymbol{\tau}_{p}^{2}-\boldsymbol{\tau}_{p}^{1}=
$$




$$
\begin{aligned}
& \boldsymbol{J}_{p}^{T}\left(\boldsymbol{J}_{t}^{T}\right)^{-1}\left(\boldsymbol{M}_{t}^{2} \ddot{\boldsymbol{q}}_{t}^{2}-\boldsymbol{M}_{t}^{1} \ddot{\boldsymbol{q}}_{t}^{1}+\boldsymbol{C}_{t}^{2} \dot{\boldsymbol{q}}_{t}^{2}-\boldsymbol{C}_{t}^{1} \dot{\boldsymbol{q}}_{t}^{1}+\boldsymbol{G}_{t}^{2}-\boldsymbol{G}_{t}^{1}\right)+\boldsymbol{M}_{p}^{2} \ddot{\boldsymbol{q}}_{p}^{2}-\boldsymbol{M}_{p}^{1} \ddot{\boldsymbol{q}}_{p}^{1}+\boldsymbol{C}_{p}^{2} \dot{\boldsymbol{q}}_{p}^{2}- \\
& \boldsymbol{C}_{p}^{1} \dot{\boldsymbol{q}}_{p}^{1}
\end{aligned}
$$

3. The patient does not exert any active joint torque voluntarily. This is important because $\boldsymbol{\tau}_{p}$ encompasses all active joint torques, there is no way to separate voluntary and involuntary actions.

Finally, we integrate $\Delta \boldsymbol{\tau}_{p}$ over the entire cycle of the movement to obtain the joint torques of the patient:

$$
\boldsymbol{\tau}_{p}=\int \frac{\Delta \tau_{p}}{\Delta t} d t
$$

With the joint torques computed, we can estimate the muscle activations.

\subsection{Experimental Setup}

As mentioned in Section 4.7, one way to validate the arm model, albeit an indirect one, is to make use of the application to demonstrate its usefulness, by correlating with the existing practices. Naturally, the next step will be to design and perform experiments with therapists and stroke patients in a clinical setting. This section describes the experiment setup.

\subsubsection{Participants and evaluators}

Fifteen patient participants (8 females, 7 males, mean age $56.9 \pm 10.4$, age range 32 - 75) participated in the study after providing informed consent. The demographic information of the participants is detailed in Table 13. The study was approved by the internal review board of Tan Tock Seng Hospital in Singapore (DSRB No.: 2015/00371), and the experiment was performed at Centre for Advanced Rehabilitation Therapeutics (CART) at the hospital. Two trained therapists (one male and one female) were involved in the experiments. During each test, only one therapist evaluated the patient participant, and two 
assistants helped to record the measurements and ensure the experiment protocols were followed closely.

Table 13: Demographic information of the participants

\begin{tabular}{cccccccc}
\hline $\begin{array}{c}\text { Parti } \\
\text { ci- } \\
\text { pant }\end{array}$ & $\begin{array}{c}\text { Gende } \\
\mathrm{r}\end{array}$ & $\begin{array}{c}\text { Ag } \\
\mathrm{e}\end{array}$ & $\begin{array}{c}\text { Heigh } \\
\mathrm{t}(\mathrm{m})\end{array}$ & $\begin{array}{c}\text { Weight } \\
(\mathrm{kg})\end{array}$ & $\begin{array}{c}\text { Type } \\
\text { of } \\
\text { Stroke }\end{array}$ & $\begin{array}{c}\text { Side of } \\
\text { Stroke }\end{array}$ & $\begin{array}{c}\text { Months } \\
\text { since stroke }\end{array}$ \\
\hline 1 & $\mathrm{~F}$ & 56 & 1.6 & 65.9 & $\mathrm{H}$ & $\mathrm{L}$ & 13 \\
2 & $\mathrm{~F}$ & 32 & 1.75 & 61 & $\mathrm{H}$ & $\mathrm{L}$ & 58 \\
3 & $\mathrm{~F}$ & 52 & 1.73 & 57 & $\mathrm{H}$ & $\mathrm{R}$ & 10 \\
4 & $\mathrm{M}$ & 53 & 1.56 & 68 & $\mathrm{I}$ & $\mathrm{L}$ & 30 \\
5 & $\mathrm{M}$ & 53 & 1.83 & 66.5 & $\mathrm{I}$ & $\mathrm{R}$ & 109 \\
6 & $\mathrm{~F}$ & 53 & 1.62 & 44.8 & $\mathrm{I}$ & $\mathrm{R}$ & 12 \\
7 & $\mathrm{M}$ & 45 & 1.81 & 78 & $\mathrm{H}$ & $\mathrm{R}$ & 17 \\
8 & $\mathrm{~F}$ & 57 & 1.58 & 65 & $\mathrm{I}$ & $\mathrm{L}$ & 12 \\
9 & $\mathrm{M}$ & 63 & 1.68 & 63 & $\mathrm{H}$ & $\mathrm{R}$ & 44 \\
10 & $\mathrm{~F}$ & 61 & 1.53 & 60 & $\mathrm{H}$ & $\mathrm{R}$ & 5 \\
11 & $\mathrm{~F}$ & 68 & 1.52 & 60.6 & $\mathrm{H}$ & $\mathrm{R}$ & 6 \\
12 & $\mathrm{M}$ & 69 & 1.8 & 80 & $\mathrm{I}$ & $\mathrm{R}$ & 3 \\
13 & $\mathrm{~F}$ & 56 & 1.56 & 55 & $\mathrm{H}$ & $\mathrm{R}$ & 3 \\
14 & $\mathrm{M}$ & 75 & 1.59 & 73.1 & $\mathrm{H}$ & $\mathrm{R}$ & 121 \\
15 & $\mathrm{M}$ & 61 & 1.71 & 75 & $\mathrm{I}$ & $\mathrm{L}$ & 5 \\
\hline \multicolumn{7}{c}{ Note: H - Haemorrhage; I - Infarct } \\
\hline
\end{tabular}

\subsubsection{Clinical testing}

The therapists performed tests of the elbow flexor spasticity on the patient's affected arm based on Modified Ashworth Scale (MAS) [34]. The patient sat on a chair with the arms resting in a relaxed position beside the body. The therapist stood beside the patient and abducted the patient's humerus to $90^{\circ}$ (or within a pain free range of shoulder movement), and flexed the elbow fully. While supporting the patient's abducted arm in position, the therapist then extended the patient's elbow fully in one second (counting “one thousand”, for example, as a guide). The therapist graded and recorded the MAS score of the patient. This assessment procedure was repeated for the patients' shoulder, wrist and thumb, to obtain a comprehensive ranking of the severity of the patients' conditions. 


\subsubsection{Instrumented testing}

The instrument testing started 5 minutes after the clinical testing to diminish any possible fatigue effect in the patient's upper limb. In addition to the recording of the height and weight of the patient participants and the therapists, the lengths of the clavicle (distance between the sternoclavicular (SC) joint and the acromioclavicular (AC) joint), the humerus (distance between the edge of the shoulder and the elbow) and the forearm (distance between the elbow and the wrist) were also measured using a measuring tape. All these measurements were used in the estimations of the inertial properties of the patient's and therapist's upper limbs using the method depicted in Section 3.6.

Three IMUs (APDM Opal ${ }^{\text {TM }}$ wireless) were attached to the patient's affected upper limb and three more to the therapist's upper limb that performed the test (always the same side as the patient's affected upper limb). The first IMU was attached to the flat portion of the sternum, just below the neck, using double sided tape or Velcro strap; the second IMU was strapped around the middle part of the upper arm; and the third IMU was strapped around the lower arm, just above the wrist (compare Figure 27). A pair of EMG electrodes (Biopac Systems Inc Data Acquisition System MP150 and EMG Amplifier EMG100C) was attached to the cleaned skin surface of the patient's biceps brachii to monitor the electromyographic activities of the muscle for comparison to the upper limb model predictions. A ground electrode was placed at a neutral site on the upper arm as a reference. 


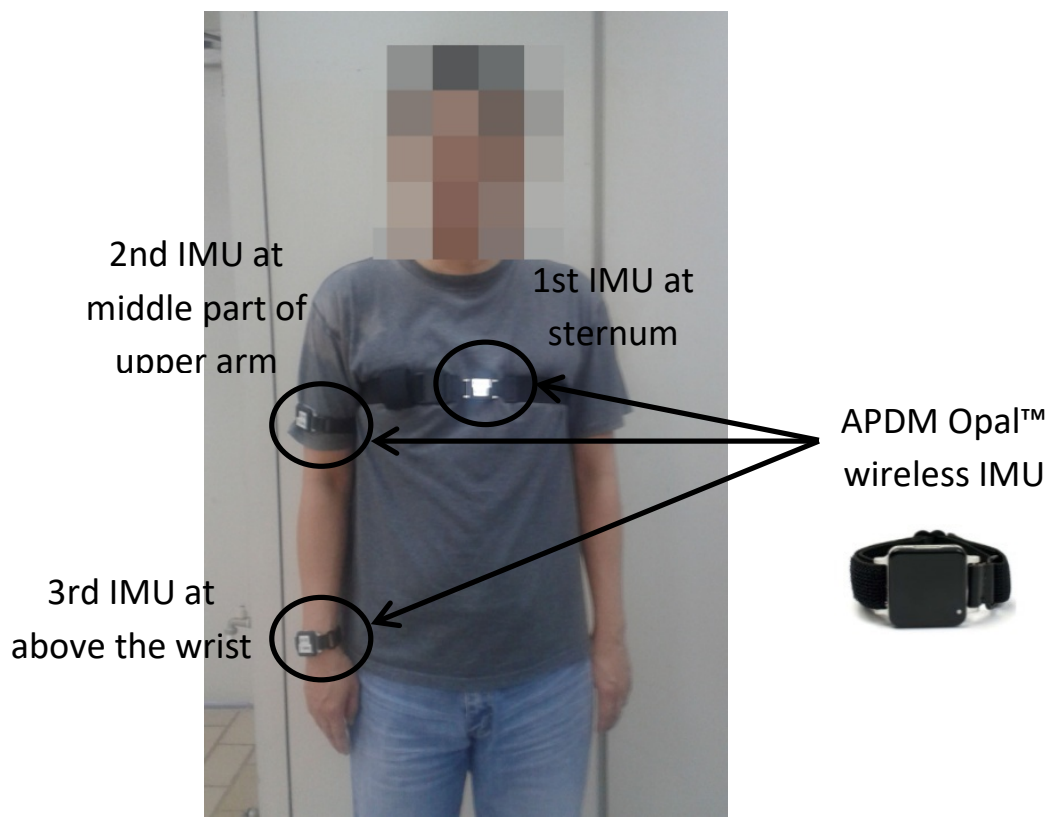

Figure 27: Placement of the three APDM Opal ${ }^{\mathrm{TM}}$ wireless IMUs on the upper limb.

The test procedure was similar to the clinical testing, except the therapist was instructed to perform the tests for slow, medium and fast speeds of motion. For the identification of the initial reference configuration of the upper limb models, the data were recorded while the patient sat on a chair with the arms resting in a relaxed position beside the body for 5 seconds before the therapist held up the patient's arm. The therapist then extended the patient's elbow in 2 to 3 seconds for slow speeds, in 1 to 2 seconds for medium speeds, and as quickly as possible without causing pain to the patient for fast speeds. For each speed range, the tests were performed four times with one minute rest after two repetitions. A total of 12 tests were carried out for each patient.

\subsubsection{Data analysis}

The EMG signal was first rectified and passed through a filter with frequency band of $20-350 \mathrm{~Hz}$. The frequency range of $20-350 \mathrm{~Hz}$ is the range where meaningful EMG signals can be captured. This range has been widely used by previous studies $[12,150,151]$. The linear envelope of the EMG signal was found using rectify-and-mean approach with window width of 1000 points. The 
magnitude of the EMG signal during the initial reference position was taken as the baseline EMG. The joint angle and the velocity at which the EMG signal went beyond 2SDs of the mean baseline defined the DSRT (DSRT_EMG) for that particular test.

The data from the IMUs, on the other hand, was used to compute the muscle activations with the upper limb model based on the procedure described in previous chapters. For instance, Figure 28 shows the resulting predicted muscle activations of six muscle groups of participant 3 at a medium speed (about $75^{\circ}$ per second). The DSRT was defined by the joint angle at which the predicted activation increased over $1 \%$ of maximum voluntary contraction (1\%MVC). We made use of the measured MVC of stroke patients with similar demography as our participants published in [152]. Two types of DSRTs were computed for each test, one was based on the activation of biceps brachii (DSRT_B) and the other was based on the first activation of any muscle that crossed the 1\%MVC threshold (DSRT_A). In the particular example shown in Figure 28 the DSRT_A was triggered by the activation of the deltoid muscle, which crossed the 1\%MVC first. The predicted TSRT of each participant was defined as the X-intercept of the linear regression on the DSRTs. For instance, Figure 29 shows the linear regression of the DSRT_Bs of participant 3 and her TSRT_B. Both DSRT and TSRT were measured in \%Motion. 


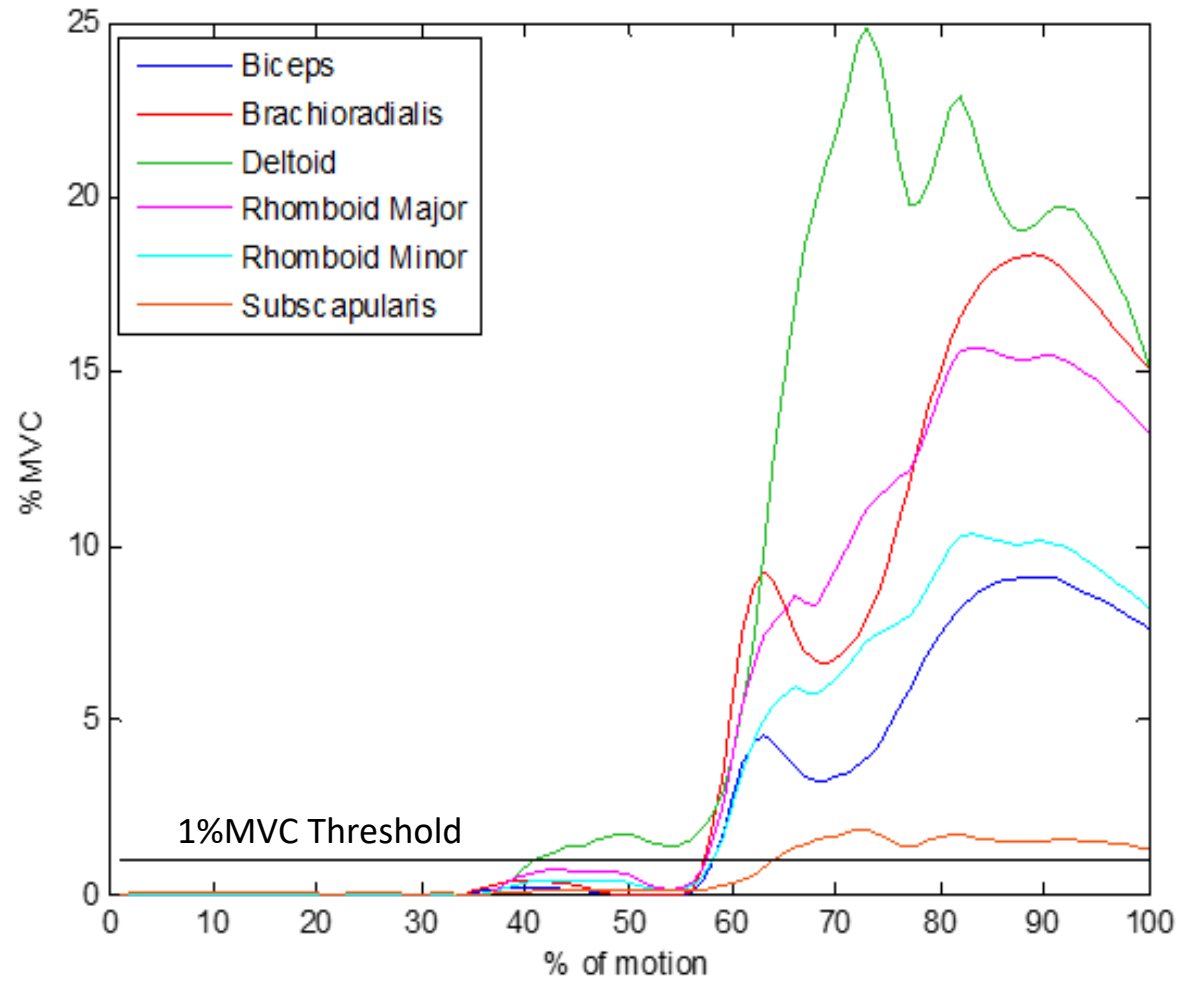

Figure 28: Muscle activations of six groups of muscles predicted by the model at a medium speed of about $75 \%$ sec. The muscle activation is computed in \%MVC and plotted against the \% completion of the extension motions. The muscles selected cover the forearm (Brachioradialis), upper arm (Biceps and Deltoid), shoulder anterior (Subscapularis) and shoulder posterior (Rhomboid Major and Minor). 


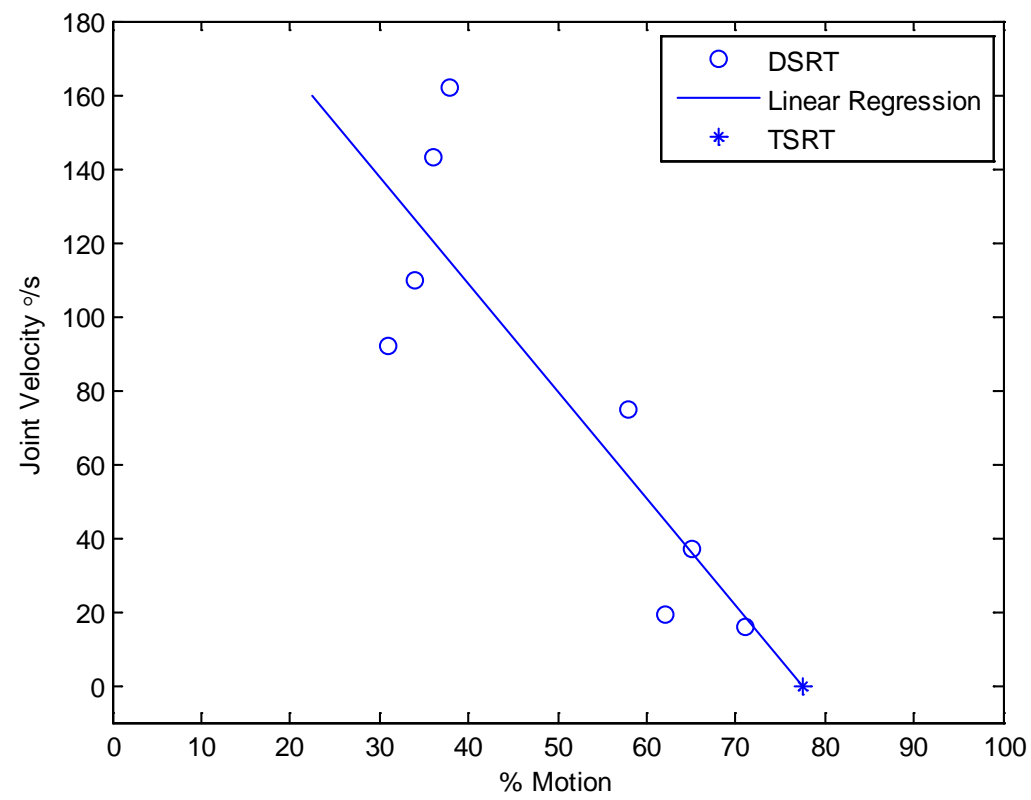

Figure 29: The linear regression of the DSRT_B of Participant 3. TSRT_B is the $\mathrm{x}$-intercept of the regression line.

\subsection{Results}

\subsubsection{Validation of model using EMG}

There is no simple and universal method to validate the muscle forces predicted by models. Most attempts at validation compare predicted muscle loading or activation patterns against EMG data [18]. Some researchers are also using EMG-triggering in the design of stroke treatment methods [153]. These reasons compel us to use DSRT_EMG as a mean of validation for DSRT_B in our experiment. However, it was a challenge to obtain meaningful EMG from some of the participants due to muscle atrophy and the difficulty in the attachment of the electrodes. Out of the 15 participants, only 5 participants (Participant 1, 3, 4, 9 and 11) provided useful EMG signals for the definition of DSRT_EMG ( $n=$ 38, where $n$ is the number of DSRTs used in the comparison). Using these DSRT_EMG and the corresponding DSRT_B, we find the mean and the standard deviation of the difference [154], DSRT_diff, between the two sets of data 
(DSRT_EMG-DSRT_B), $\bar{d}$ and $s$, to be $-4.21 \%$ Motion and $14.36 \%$ Motion, respectively. From this, we compute the 95\% confidence intervals for DSRT_diff using $t$-distribution [154] to be -8.93 to $0.51 \%$ Motion. The profiles of model predicted muscle activation and the EMG signal through the full cycle of motion are also compared by computing the cross-correlations. One such comparison is shown in Figure 30. The linear envelope of the EMG signal is normalized such that the 2SDs above the mean baseline coincides with the 1\%MVC. The mean cross-correlation of all 38 tests, $\bar{c}$, is 0.707 (with $\mathrm{SD}=0.077$ ).

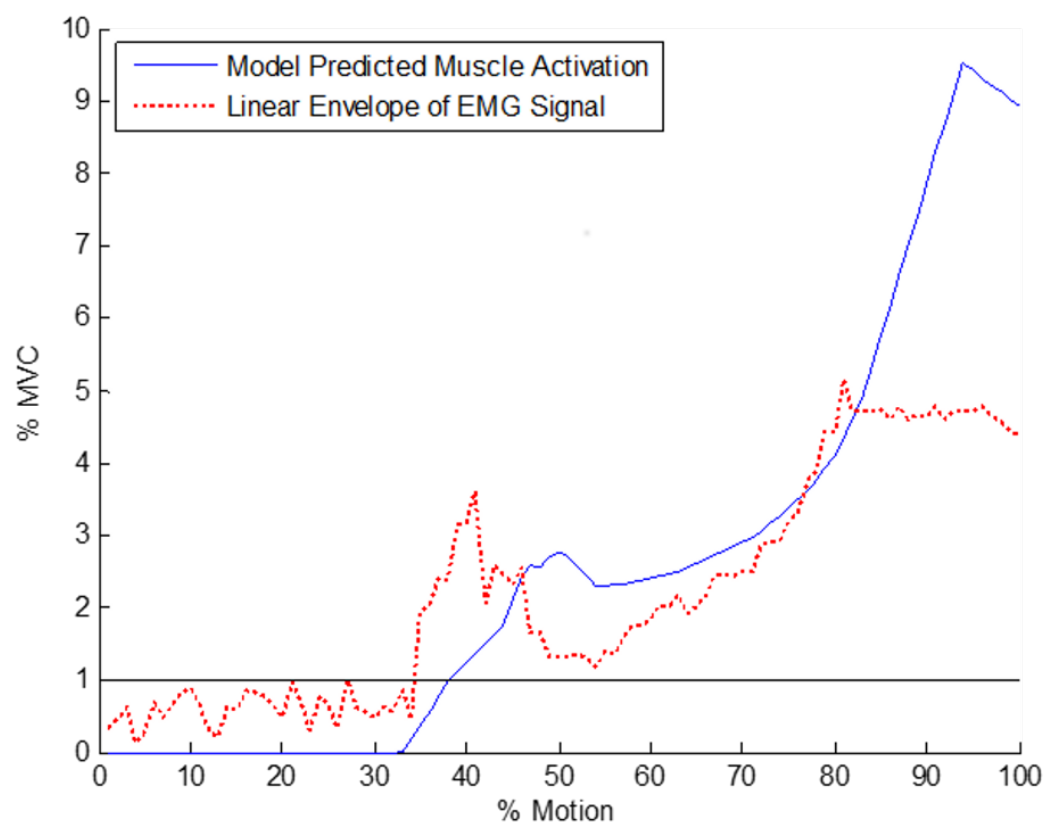

Figure 30: Model prediction of muscle activation and linear envelope of EMG signal of biceps brachii of Participant 3 at a speed of about $162 \%$ sec. The EMG envelope was normalized such that the 2SDs above the mean baseline coincides with the 1\%MVC of the model prediction. The cross-correlation between the two profiles for this particular test is 0.84 .

\subsubsection{Comparison between model predictions and clinical results}

Although the current practice of using MAS to assess the severity of spasticity in patients suffers from some issues that affect its reliability and accuracy [11], it is still widely accepted as the standard assessment method. It is therefore important for any new assessment method to benchmark its predictions against results based 
on MAS. To test if the assessment of spasticity using the upper limb model is a viable alternative to using MAS, we examine the correlation between the two methods. However, there is no quantifiable parameter for the comparison between the two methods. As such, we compare the rankings in the severity of spasticity in the patients as predicted by the two methods.

Two sets of TSRT (computed in term of \%Motion) for all 15 participants, one based on the activations of biceps brachii (TSRT_B), and the other (TSRT_A) on the earliest activations of any of the six groups of muscles (brachioradalis, biceps brachii, deltoid, subscapularis, rhomboid major and rhomboid minor), are being ranked from the lowest TSRT (least severe case) to the highest TSRT (most severe case). Similarly, the severity of spasticity of the participants assessed clinically is also ranked with respect to their MAS scores $(0,1,1+, 2,3$ or 4$)$. The participants are first ranked using the MAS scores for elbow extension tests. The ties are settled using MAS scores for shoulder, wrist and thumb, in that sequence. The MAS scores for shoulder, wrist and thumb do not directly measure the spasticity of the elbow flexors, but they are also used clinically as indicators to the severity of spasticity. Due to the ambiguity in the coarse scale of MAS, we need some indicators for us to meaningfully rank the severity of the participants. After consulting the therapists, we decided to make use of the scores. The results are summarized in Table 14.

We use Spearman's rank correlation and correlation testing via t-test to test the null hypothesis that there is no correlation between the severity rankings of (a) TSRT_B and MAS; and (b) TSRT_A and MAS. The results are presented in Table 15. The two tests have their null hypothesis rejected, showing correlations between the model predictions and the clinical assessments. The model predictions and assessment using MAS have very small $p$-value (0.0003 for TSRT_B and MAS, and 0.0002 for TSRT_A and MAS), showing high correlations between the two methods. 
Table 14: The model prediction for TSRTs and the clinical assessment results in Modified Ashworth Scale (MAS). TSRT Biceps uses the activation of biceps brachii while TRST Others uses the earliest activation of any of the six groups of muscles (brachioradalis, biceps brachii, deltoid, subscapularis, rhomboid major and rhomboid minor). The model predictions are ranked based on the TSRT (in \%Motion). The MAS is ranked first by the score $(0,1,1+, 2,3,4)$ for elbow extension tests. The ties are settled using MAS scores for shoulder, wrist and thumb, in that sequence.

\begin{tabular}{|c|cc|cc|ccccc|}
\hline Sub & $\begin{array}{c}\text { TSRT_B } \\
\% \text { Motio } \\
\mathrm{n}\end{array}$ & $\begin{array}{c}\text { Ran } \\
\mathrm{k}\end{array}$ & $\begin{array}{c}\text { TSRT_A } \\
\% \text { Motio } \\
\mathrm{n}\end{array}$ & $\begin{array}{c}\text { Ran } \\
\mathrm{k}\end{array}$ & $\begin{array}{c}\text { MAS } \\
-\mathrm{E} \\
\text { Score }\end{array}$ & $\begin{array}{c}\text { MAS } \\
-\mathrm{S} \\
\text { Score }\end{array}$ & $\begin{array}{c}\text { MAS } \\
-\mathrm{W} \\
\text { Score }\end{array}$ & $\begin{array}{c}\text { MAS } \\
\text { Score }\end{array}$ & $\begin{array}{c}\text { Ran } \\
\mathrm{k}\end{array}$ \\
\hline 1 & 85.02 & 7 & 70.91 & 10 & 1 & 0 & $1+$ & $1+$ & 9.5 \\
2 & 49.98 & 12 & 48.53 & 12 & 1 & 0 & 1 & $1+$ & 7 \\
3 & 76.49 & 10 & 75.50 & 8 & 0 & 0 & 0 & 0 & 2.5 \\
4 & 94.42 & 4 & 93.12 & 4 & 0 & 0 & 1 & 0 & 5 \\
5 & 42.46 & 14 & 38.64 & 14 & $1+$ & 1 & $1+$ & 1 & 15 \\
6 & 109.42 & 1 & 109.42 & 3 & 0 & 0 & 0 & 0 & 2.5 \\
7 & 89.90 & 5 & 89.92 & 5 & 1 & 0 & 0 & 1 & 6 \\
8 & 58.65 & 11 & 64.15 & 11 & $1+$ & 1 & 1 & 1 & 14 \\
9 & 88.84 & 6 & 89.59 & 6 & 1 & 0 & $1+$ & $1+$ & 9.5 \\
10 & 105.54 & 2 & 109.52 & 2 & 0 & 0 & 0 & 0 & 2.5 \\
11 & 80.64 & 9 & 74.38 & 9 & 1 & $1+$ & 1 & 0 & 11 \\
12 & 103.99 & 3 & 109.69 & 1 & 0 & 0 & 0 & 0 & 2.5 \\
13 & 44.26 & 13 & 39.86 & 13 & $1+$ & 1 & 1 & 0 & 13 \\
14 & 27.33 & 15 & 28.30 & 15 & 1 & 0 & $1+$ & 1 & 8 \\
15 & 82.23 & 8 & 85.41 & 7 & $1+$ & 0 & 0 & 0 & 12 \\
\hline
\end{tabular}

Note: TSRT_B - TSRT based on biceps; TSRT_A - TSRT based on any of the six muscles; MAS-E - Modified Ashworth Scale on elbow; MAS-S - MAS on shoulder; MAS-W - MAS on wrist; MAS-T - MAS on thumb. For tied rankings, the average is used. 
Table 15: The results of the null hypothesis, $H_{0}$, that the model prediction of the severity of spasticity via TSRT has no correlation to the clinical assessments via MAS. The hypothesis testing makes use of Spearman's rank correlation test via $t$ test, with $n=15$ and significance level, $\alpha=0.05$. The null hypothesis is rejected if $p$-value $<\alpha$.

\begin{tabular}{cccccccc}
\hline $\begin{array}{c}\text { Comparison } \\
\text { Pair }\end{array}$ & $n$ & $\begin{array}{c}\text { Spearman’s } \\
\text { rho, } r\end{array}$ & $\alpha$ & t-critical & $t$ & $\begin{array}{c}p \text { - } \\
\text { value }\end{array}$ & $\begin{array}{c}\text { Reject } \\
H_{0} ?\end{array}$ \\
\hline $\begin{array}{c}\text { TSRT_B } \\
\text { and MAS }\end{array}$ & 15 & 0.807 & 0.05 & 2.16 & 4.93 & 0.0003 & Yes \\
$\begin{array}{c}\text { TSRT_A } \\
\text { and MAS }\end{array}$ & 15 & 0.821 & 0.05 & 2.16 & 5.19 & 0.0002 & Yes \\
\hline
\end{tabular}

Note: TSRT_B - TSRT based on biceps; TSRT_A - TSRT based on any of the six muscles; MAS -Modified Ashworth Scale on elbow.

\subsection{Discussion}

\subsubsection{Validation of the Model using EMG and Its Implications}

From the results, we see that the 95\% confidence interval (-8.93 \%Motion and $0.51 \%$ Motion as the lower and upper bounds respectively) for the DSRT_diff (the difference between DSRT_EMG and DSRT_B) reveals that EMG tracking tends to provide a lower DSRT than that predicted by the model. The standard deviation of the difference at $14.36 \%$ is significant. Two of the possible reasons for this are: (a) the triggering thresholds of the EMG signals set at 2 SD of the baseline signal is too low; or (b) the 1\%MVC threshold for the model prediction is set too high. The former is a standard practice which is based on the assumption that the baseline signal is white noise and it was not caused by muscle activation $[11,12]$. The $1 \%$ MVC threshold for the model prediction was chosen by examining the muscle activation plots like the one shown in Figure 27, as it is necessary to exclude possible activation noise, which is more significant at low muscle activation [155], during the experiments. Lowering the threshold would risk taking noise as the actual involuntary muscle activation. However, the correlation coefficient $(\bar{c}=0.707)$ between the activation profile of the model's 
prediction and the EMG envelope indicates that the two correlate well. This is a better indicator for the similarity between the two methods because it compares the activations throughout the full motion and it is also independent of the chosen thresholds. Considering that the model uses kinematic data, which were fundamentally different from EMG signal, to predict the muscle activations, the high correlation provides confidence that the new method is performing well.

Using EMG to evaluate spasticity has its own issues such as diffusion effects of the skin [60, 61], placement of the electrodes [45], and muscle atrophy of elderly or stroke patients [11], that need to be addressed. There are two advantages the upper limb model has over EMG to measure DSRTs. First, non-invasive EMG has the limitation of measuring only the large and superficial muscles and the complexity of spasticity cannot be fully captured. The muscular upper limb model provides a platform for the understanding of the relationship between involuntary resistance to movement and the activations of deep muscles. Secondly, the EMG sensors have to be used together with motion sensors, where the former identifies the muscle stretch reflexes and the latter measures the velocity. The costs and trainings needed to operate these instruments may hinder the promotion of using objective assessment methods in general. The model introduced in this paper uses only the IMUs as sensors and requires only simple protocols to use. The time needed for the therapist to put on the sensors on the patient's body and then on her own body is about 5 mins (with the help of another person, this can reduce to about 2 to 3 mins). This short set-up time is not likely to significantly lengthen the assessment routine.

As the data collected from the experiment are only from five participants, there are too few data for this comparison to be a conclusive validation. More EMG data will be collected in future experiments to strengthen the validation. 


\subsubsection{The Correlation between Model Prediction and the Clinical Assessments and Its Implications}

The model predictions correlate very well with the MAS assessments performed by trained therapists in terms of the severity ranking among the patients. This is not a surprise because both the model and the therapist are trying to identify the "catch" (the moment when the involuntary resistance sets in). The high correlation provides confidence in the model as a complementary tool to the existing practice. The particiapnts recruited for the experiments in this study were less severe stroke patients who showed mild to moderate conditions and their MAS scores for the elbow extension only ranged between 0 and 1+ (Table 14). The ranking of the MAS scores has to be aided by using the MAS scores for other parts of the upper limb. The simplicity in the Modified Ashworth Scale is the reason for its popularity and at the same time also its most criticized attribute [11, 156]. In particular, it is not easy to identify significant differences between the scores $1,1+$ and 2 [156]. If limited resources for rehabilitation were to be distributed among the patients according to the severity in spasticity, or one has to design rehabilitation programs for the patients based on the assessments, the lack of fine distinctions in the MAS score poses a problem. The model predictions can be used in these cases to differentiate the level of severity of spasticity among the patients.

\subsection{Future Works for Spasticity Assessments}

The results from the experiment showed that there is a good correlation between the spasticity severity predictions using the model and from assessment by MAS. This provides some confidence that we are on the right track in developing a complementary assessment method to the current clinical practice. For the current study, due to experimental constraints, the MVCs were not directly measured. In the future experiments, the measurement of MVC of participants will be included in the experiment protocols. Moving forward, we will engage clinicians to use the system alongside with their daily assessment routine, collect 
more data, and make improvements to the model and protocols based on the feedback from the users. To make it a more viable and hassle-free tool that the clinicians are willing to use, a few features can be added in the next stage of development. First, to simplify the set up procedure and reduce discomfort for the patient, the three sensors can be incorporated into a wearable sensor suit. The criteria for the design of such a suit are the movement of the user should not be constrained, and the sensors can be tightly secured to the various upper limb segments. Second, data logging and analysis processes to be automated. Currently, these two processes are done manually post-experiment by the researchers instead of the clinicians. They have to be automated since the purpose of the system is to be part of the daily assessment routine. 


\section{Conclusion and Future Works}

\subsection{Conclusion}

The current trend for assessment during stroke rehabilitation is moving towards objective measurements to complement the subjective assessments by therapists. These objective measurements are important for at least three reasons. First, objective measurement provides a platform to resolve the subjectivity problem in many current methods where the interrater reliability is low. Second, the development of a standard protocol for assessment using measurement from sensors would reduce the reliance on experienced therapists to perform the routine assessments and ease the manpower shortage problem. Third, one good way to increase the efficacy of rehabilitation program is to customize the program based on the assessed condition of the patient. Objective measurements can provide physiological information that otherwise hard to obtain using subjective assessment. One parameter that can be used as an indicator in the assessment is the muscle force. As muscle forces are difficult, if possible, to measure directly, a popular alternative method is to estimate the forces using musculoskeletal models. However, there is still more work to be done before the current modelbased methods for estimation of muscle forces can be used as a clinical tool in stroke assessment. In this research, we developed a musculoskeletal model for the human upper limb with the objective of bridging this gap, providing a tool for objective assessment in stroke rehabilitation that can be used to complement the current practices. To demonstrate the capability of the model, we use it in the application of quantifying the severity of spasticity by measuring the tonic stretch reflex threshold. The main contributions of this research are summarized in the following points:

- Shoulder model: A hybrid method to fuse the ambulatory data from sensors with shoulder rhythm model data.

The kinematics of the shoulder complex is generally considered as the most difficult to measure when compared to other joints. The movements 
of the shoulder are the complex relative motions (which are termed "shoulder rhythm") among the three bones: clavicle, scapula and humerus. Non-invasive measurement, where a sensor or marker is attached to the skin, is often contaminated with the noise caused by soft tissue movements when the movement of the humerus is large. Invasive method, where markers are surgically attached to the scapula, on the other hand, provides accurate measurements of the orientations of scapula and clavicle with respect to the position of the humerus, in the form of shoulder rhythm. However, it is unable to capture subtle movements like shrugging of shoulder. The hybrid method introduced in this research, combines the strengths of both invasive and non-invasive measurements. This is done through a selection of data either from direct non-invasive measurement, when the movement of the humerus is small, or from the shoulder rhythm model constructed using invasive measurement, when the movement of the humerus is large. This hybrid method provides a mean to measure the kinematics of the shoulder complex in a clinical setting.

- Elbow model: A method to compute the orientation of the elbow joint using product of exponential (POE) and optimization to eliminate the need for orthogonality assumption and introduction of carry angle.

The elbow is a two degree-of-freedom joint and the two axes of rotation (flexion/extension axis and supination/pronation axis) are, in general, non-orthogonal. In addition, the flexion/extension axis is not orthogonal to the longitudinal axis of the humerus too. When using sensors to measure the elbow orientation, it is common to assume orthogonality between the two axes and decompose the rotation matrix of the forearm relative to the humerus. This introduces a third angle called the carry angle, which is often treated as a constant angle, although it varies with the elbow orientation. In this research, we derived a method to obtain the 
flexion/extension angle and the supination/pronation angle from the rotation matrix using product of exponential expression and an optimization routine, without the need to assume orthogonality and to introduce the carry angle. By incorporating this decomposition method in our musculoskeletal model, we can improve the accuracy of the estimation of muscle forces because the lines of action of the muscles are more accurately represented.

- Musculoskeletal model of the human upper limb: Developed to be useful clinically for the assessment of stroke rehabilitation.

The main contribution of this research is the development of a musculoskeletal model of the human upper limb for the assessment of stroke rehabilitation. The model is specifically developed to be useful clinically as a tool for therapists to complement the existing practices. Building on the shoulder model and elbow model mentioned above, in addition with a simple protocol to estimate the inertial properties of the upper limb segments, the biomechanical model computes the joint dynamics from the kinematic data measured by inertial sensors. The joint torques are used in the musculoskeletal model to estimate the muscle forces and activations. The estimated muscle forces can be used as indicators in the assessment of the efficacy of rehabilitation programs, and also as a mean of validation for the current subjective assessment methods. The key features of our model that make it potentially a viable clinical tool for stroke rehabilitation assessment are:

(a) The model is designed to only require input parameters that can be readily obtained in a clinical setting without the need of a trained therapist and expensive equipment.

(b) The operating protocol, including setting up the sensors, does not require any training for the operator and it takes only a few minutes. 
(c) The measurement of the kinematics of the upper limb segments can be performed simultaneously with the usual assessment routines, minimizing the discomfort for the patients.

(d) Estimated muscle forces and activations from the model can be used as a validation for many different stroke assessment tools, including task-based and reaction-based tests.

These key features lower the hurdle for clinicians to adopt the new tool by introducing non-drastic changes to the current practices. We believe such an objective assessment tool, which is meant to complement the current common tools, is only useful if the clinicians or therapists are willing to use it.

- Application: Using the model to assess the severity of spasticity by measuring the tonic stretch reflex threshold.

The upper limb model was used to quantify the tonic stretch reflex threshold (TSRT) in stroke patients to assess the severity of spasticity. The measurement of the kinematics of the upper limb was carried out concurrently with the Modified Ashworth Scale (MAS) assessment. The results showed that the rankings of the severity of spasticity predicted by using the model via TSRT have high correlation with the assessments by trained therapists. This means that the model has the potential to be used as a validation tool to MAS assessment, which has interrater reliability issues.

\subsection{Future Works}

The main objective of this research work is to offer clinicians and therapists working on stroke rehabilitation a feasible alternative tool that provides objective assessment. Although we have demonstrated that the model is capable of predicting severity of spasticity with comparable accuracy to the current clinical practice, there are possible future improvements to be done in three areas: 


\section{(a) Accuracy of the upper limb model}

\section{- Muscle origin and insert positions}

The accuracy of the muscle origin and insert positions can be improved if they are adjusted according to the relative segment lengths between the subject and the reference data source.

- Inertial properties of the upper limb segments

The published data for the inertial properties we used currently were from publications focusing on the demography of western societies. Incorporating data from an Asian population would improve the accuracy of the prediction when the model is used in Asian countries like Singapore.

(b) Operation procedures

- Design of a wearable sensor suit for the upper limb

Currently, each IMU is separately attached to the patient's body using Velcro straps. To reduce the discomfort experienced by the patient, and speed up the preparation process, the next step is to design a sensor suit that attaches the sensors in position when the patient wears it on the affect upper limb. The suit would also minimize the risk of the clinician strapping the sensors at the wrong position or orientation.

- Incorporating measurement of maximum voluntary contraction (MVC) of subjects into the protocol

For the current study, due to experimental constraints, the MVCs were not directly measured. In the future experiments, the measurement of MVC of participants will be included in the experiment protocols. 


\section{Appendix A: List of muscles and their Obstacle- set parameters}

\begin{tabular}{|c|c|c|c|}
\hline No. & Muscle & Obstacle & Frame \\
\hline \multirow{2}{*}{1} & \multirow{2}{*}{ Subclavius (SBCL) } & Origin & Thorax \\
\hline & & Insert & Clavicle \\
\hline \multirow{3}{*}{$2 \mathrm{a}$} & \multirow{3}{*}{$\begin{array}{l}\text { Serratus anterior } \\
\text { (superior) (SRAS) }\end{array}$} & Origin & Thorax \\
\hline & & Single cylinder & Thorax \\
\hline & & Insert & Scapula \\
\hline \multirow{3}{*}{$2 b$} & \multirow{3}{*}{$\begin{array}{l}\text { Serratus anterior } \\
\text { (middle) (SRAM) }\end{array}$} & Origin & Thorax \\
\hline & & Single cylinder & Thorax \\
\hline & & Insert & Scapula \\
\hline \multirow{3}{*}{$2 c$} & \multirow{3}{*}{$\begin{array}{l}\text { Serratus anterior } \\
\text { (inferior) } \\
\text { (SRAI) }\end{array}$} & Origin & Thorax \\
\hline & & Single cylinder & Thorax \\
\hline & & Insert & Scapula \\
\hline \multirow{3}{*}{ За } & \multirow{3}{*}{$\begin{array}{c}\text { Trapezius (C1-C6) } \\
\text { (TRPC) }\end{array}$} & Origin & Thorax \\
\hline & & Single cylinder & Thorax \\
\hline & & Insert & Scapula \\
\hline \multirow{3}{*}{$3 b$} & \multirow{3}{*}{$\begin{array}{l}\text { Trapezius (C7) } \\
\text { (TRPC2) }\end{array}$} & Origin & Thorax \\
\hline & & Single cylinder & Thorax \\
\hline & & Insert & Scapula \\
\hline \multirow{3}{*}{ 3c } & \multirow{3}{*}{$\begin{array}{l}\text { Trapezius (T1) } \\
\text { (TRPT) }\end{array}$} & Origin & Thorax \\
\hline & & Single cylinder & Thorax \\
\hline & & Insert & Scapula \\
\hline \multirow{3}{*}{$3 d$} & \multirow{3}{*}{$\begin{array}{c}\text { Trapezius (T2-T7) } \\
\text { (TRPT2) }\end{array}$} & Origin & Thorax \\
\hline & & Single cylinder & Thorax \\
\hline & & Insert & Scapula \\
\hline \multirow{2}{*}{4} & \multirow{2}{*}{$\begin{array}{c}\text { Levator scapulae } \\
\text { (LVS) }\end{array}$} & Origin & Thorax \\
\hline & & Insert & Scapula \\
\hline \multirow{3}{*}{5} & \multirow{3}{*}{$\begin{array}{l}\text { Rhomboid minor } \\
\text { (RMN) }\end{array}$} & Origin & Thorax \\
\hline & & Single cylinder & Thorax \\
\hline & & Insert & Scapula \\
\hline \multirow{3}{*}{$6 a$} & \multirow{3}{*}{$\begin{array}{l}\text { Rhomboid major } \\
\text { (T1-T2) } \\
\text { (RMJ) }\end{array}$} & Origin & Thorax \\
\hline & & Single cylinder & Thorax \\
\hline & & Insert & Scapula \\
\hline \multirow{3}{*}{$6 b$} & \multirow{3}{*}{$\begin{array}{l}\text { Rhomboid major } \\
\text { (T3-T4) } \\
\text { (RMJ2) }\end{array}$} & Origin & Thorax \\
\hline & & Single cylinder & Thorax \\
\hline & & Insert & Scapula \\
\hline \multirow{2}{*}{7} & \multirow{2}{*}{$\begin{array}{l}\text { Pectoralis minor } \\
(\mathrm{PMN})\end{array}$} & Origin & Thorax \\
\hline & & Insert & Scapula \\
\hline & Pectoralis major & Origin & Clavicle \\
\hline $8 a$ & (clavicular) & Single cylinder & Thorax \\
\hline & (PMJC) & Insert & Humerus \\
\hline & Pectoralis major & Origin & Thorax \\
\hline $8 b$ & (sternal) & Single cylinder & Thorax \\
\hline & (PMJS) & Insert & Humerus \\
\hline
\end{tabular}




\begin{tabular}{|c|c|c|c|}
\hline \multirow{3}{*}{$8 c$} & \multirow{3}{*}{$\begin{array}{c}\text { Pectoralis major } \\
\text { (ribs) } \\
\text { (PMJR) }\end{array}$} & Origin & Thorax \\
\hline & & Single cylinder & Thorax \\
\hline & & Insert & Humerus \\
\hline \multirow{4}{*}{$9 a$} & \multirow{4}{*}{$\begin{array}{l}\text { Latissimus dorsi } \\
\text { (thoracic) } \\
\text { (LTDT) }\end{array}$} & Origin & Thorax \\
\hline & & \multirow{2}{*}{ Double Cylinder } & Thorax \\
\hline & & & Scapula \\
\hline & & Insert & Humerus \\
\hline \multirow{4}{*}{$9 b$} & \multirow{4}{*}{$\begin{array}{l}\text { Latissimus dorsi } \\
\text { (lumbar) } \\
\text { (LTDL) }\end{array}$} & Origin & Thorax \\
\hline & & \multirow{2}{*}{ Double cylinder } & Thorax \\
\hline & & & Humerus \\
\hline & & Insert & Humerus \\
\hline \multirow{4}{*}{ 9c } & \multirow{4}{*}{$\begin{array}{l}\text { Latissimus dorsi } \\
\text { (iliac) } \\
\text { (LTDI) }\end{array}$} & Origin & Thorax \\
\hline & & \multirow{2}{*}{ Double cylinder } & Thorax \\
\hline & & & Humerus \\
\hline & & Insert & Humerus \\
\hline \multirow{4}{*}{$10 \mathrm{a}$} & \multirow{4}{*}{$\begin{array}{c}\text { Deltoid (clavicular) } \\
\text { (DLTC) }\end{array}$} & Origin & Clavicle \\
\hline & & Sphere-capped cylinder & Scapula \\
\hline & & Via & Humerus \\
\hline & & Insert & Humerus \\
\hline \multirow{4}{*}{$10 \mathrm{~b}$} & \multirow{4}{*}{$\begin{array}{l}\text { Deltoid (acromial) } \\
\text { (DLTA) }\end{array}$} & Origin & Scapula \\
\hline & & Sphere-capped cylinder & Scapula \\
\hline & & Via & Humerus \\
\hline & & Insert & Humerus \\
\hline \multirow{5}{*}{ 10c } & \multirow{5}{*}{$\begin{array}{l}\text { Deltoid (scapular) } \\
\text { (DLTS) }\end{array}$} & Origin & Scapula \\
\hline & & Via1 & Scapula \\
\hline & & Sphere-capped cylinder & Scapula \\
\hline & & Via2 & Humerus \\
\hline & & Insert & Humerus \\
\hline \multirow{4}{*}{11} & \multirow{4}{*}{$\begin{array}{l}\text { Supraspinatus } \\
\text { (SUPR) }\end{array}$} & Origin & Scapula \\
\hline & & Via1 & Scapula \\
\hline & & Via2 & Humerus \\
\hline & & Insert & Humerus \\
\hline \multirow{3}{*}{12} & \multirow{3}{*}{$\begin{array}{l}\text { Infraspinatus } \\
\text { (INFR) }\end{array}$} & Origin & Scapula \\
\hline & & Sphere-capped cylinder & Scapula \\
\hline & & Insert & Humerus \\
\hline \multirow{4}{*}{13} & \multirow{4}{*}{$\begin{array}{l}\text { Subscapularis } \\
\quad \text { (SBSC) }\end{array}$} & Origin & Scapula \\
\hline & & Via & Scapula \\
\hline & & Single cylinder & Scapula \\
\hline & & Insert & Humerus \\
\hline \multirow{4}{*}{14} & & Origin & Scapula \\
\hline & Teres minor & Via & Scapula \\
\hline & $(\mathrm{TMN})$ & Sphere-capped cylinder & Humerus \\
\hline & & Insert & Humerus \\
\hline & & Origin & Scapula \\
\hline 15 & Teres major & Single cylinder & Scapula \\
\hline & & Insert & Humerus \\
\hline & & & \\
\hline
\end{tabular}




\begin{tabular}{|c|c|c|c|}
\hline \multirow{4}{*}{16} & \multirow{4}{*}{$\begin{array}{c}\text { Coracobrachialis } \\
\text { (CRCB) }\end{array}$} & Origin & Scapula \\
\hline & & Single cylinder & Scapula \\
\hline & & Via & Humerus \\
\hline & & Insert & Humerus \\
\hline \multirow{4}{*}{$17 \mathrm{a}$} & \multirow{4}{*}{$\begin{array}{c}\text { Triceps brachii } \\
\text { (long) } \\
\text { (TRCL) }\end{array}$} & Origin & Scapula \\
\hline & & \multirow{2}{*}{ Double cylinder } & Humerus \\
\hline & & & Humerus \\
\hline & & Insert & Forearm \\
\hline \multirow{4}{*}{$17 \mathrm{~b}$} & \multirow{4}{*}{$\begin{array}{l}\text { Triceps brachii } \\
\text { (medial) } \\
\text { (TRCM) }\end{array}$} & Origin & Humerus \\
\hline & & Via & Humerus \\
\hline & & Single cylinder & Humerus \\
\hline & & Insert & Forearm \\
\hline \multirow{5}{*}{$17 \mathrm{c}$} & \multirow{5}{*}{$\begin{array}{l}\text { Triceps brachii } \\
\text { (lateral) } \\
\text { (TRCT) }\end{array}$} & Origin & Humerus \\
\hline & & Via1 & Humerus \\
\hline & & Single cylinder & Humerus \\
\hline & & Via2 & Forearm \\
\hline & & Insert & Forearm \\
\hline \multirow{4}{*}{$18 \mathrm{a}$} & \multirow{4}{*}{$\begin{array}{l}\text { Biceps brachii } \\
\text { (short) } \\
\text { (BICS) }\end{array}$} & Origin & Scapula \\
\hline & & \multirow{2}{*}{ Double cylinder } & Scapula \\
\hline & & & Humerus \\
\hline & & Insert & Forearm \\
\hline \multirow{5}{*}{$18 \mathrm{~b}$} & \multirow{5}{*}{$\begin{array}{l}\text { Biceps brachii } \\
\text { (long) } \\
\text { (BICL) }\end{array}$} & Origin & Scapula \\
\hline & & Sphere & Humerus \\
\hline & & Via & Humerus \\
\hline & & Single cylinder & Humerus \\
\hline & & Insert & Forearm \\
\hline \multirow{4}{*}{19} & \multirow{4}{*}{$\begin{array}{l}\text { Brachialis } \\
\text { (BRA) }\end{array}$} & Origin & Humerus \\
\hline & & Via & Humerus \\
\hline & & Single cylinder & Humerus \\
\hline & & Insert & Forearm \\
\hline \multirow{6}{*}{20} & \multirow{6}{*}{$\begin{array}{l}\text { Brachioradialis } \\
\text { (BRD) }\end{array}$} & Origin & Humerus \\
\hline & & Via1 & Humerus \\
\hline & & & Humerus \\
\hline & & Double cylinder & Forearm \\
\hline & & Via2 & Forearm \\
\hline & & Insert & Forearm \\
\hline \multirow{3}{*}{21} & \multirow{3}{*}{$\begin{array}{l}\text { Supinator } \\
\text { (SUP) }\end{array}$} & Origin & Humerus \\
\hline & & Via & Forearm \\
\hline & & Insert & Forearm \\
\hline \multirow{3}{*}{22} & \multirow{3}{*}{$\begin{array}{l}\text { Pronator } \\
\text { (PRO) }\end{array}$} & Origin & Humerus \\
\hline & & Via & Forearm \\
\hline & & Insert & Forearm \\
\hline
\end{tabular}




\section{References}

[1] N. Venketasubramanian and C. Chen, "Burden of stroke in Singapore," International Journal of Stroke, vol. 3, pp. 51-54, 2008.

[2] H. I. Krebs, J. Palazzolo, L. Dipietro, M. Ferraro, J. Krol, K. Rannekleiv, et al., "Rehabilitation robotics: Performance-based progressive robotassisted therapy," Autonomous Robots, vol. 15, pp. 7-20, 2003.

[3] N. HOGAN, "Skeletal muscle impedance in the control of motor actions," Journal of mechanics in medicine and biology, vol. 2, pp. 359-373, 2002.

[4] P. Lum, D. Reinkensmeyer, R. Mahoney, W. Z. Rymer, and C. Burgar, "Robotic devices for movement therapy after stroke: current status and challenges to clinical acceptance," Topics in stroke rehabilitation, vol. 8, pp. 40-53, 2002.

[5] K. K. Ang, C. Guan, K. S. G. Chua, B. T. Ang, C. Kuah, C. Wang, et al., "Clinical study of neurorehabilitation in stroke using EEG-based motor imagery brain-computer interface with robotic feedback," in Engineering in Medicine and Biology Society (EMBC), 2010 Annual International Conference of the IEEE, 2010, pp. 5549-5552.

[6] K. K. Ang, C. Guan, K. Sui Geok Chua, B. T. Ang, C. Kuah, C. Wang, et al., "A clinical study of motor imagery-based brain-computer interface for upper limb robotic rehabilitation," in Engineering in Medicine and Biology Society, 2009. EMBC 2009. Annual International Conference of the IEEE, 2009, pp. 5981-5984.

[7] C. Metcalf, J. Adams, J. Burridge, V. Yule, and P. Chappell, "A review of clinical upper limb assessments within the framework of the WHO ICF," Musculoskeletal Care, vol. 5, pp. 160-173, 2007.

[8] P. Langhorne, F. Coupar, and A. Pollock, "Motor recovery after stroke: a systematic review," The Lancet Neurology, vol. 8, pp. 741-754, 2009.

[9] S. E. team. (2013). StrokEngine-Assess. Available: http://strokengine.ca/assess/index-en.html

[10] R. R. Young, "Spasticity: a review," Neurology, vol. 44, pp. S12-20, 1994.

[11] F. Biering-Sørensen, J. Nielsen, and K. Klinge, "Spasticity-assessment: a review," Spinal Cord, vol. 44, pp. 708-722, 2006.

[12] A. Calota, A. G. Feldman, and M. F. Levin, "Spasticity measurement based on tonic stretch reflex threshold in stroke using a portable device," Clinical Neurophysiology, vol. 119, pp. 2329-2337, 2008.

[13] K. S. Kim, J. H. Seo, and C. G. Song, "Portable measurement system for the objective evaluation of the spasticity of hemiplegic patients based on the tonic stretch reflex threshold," Medical engineering \& physics, vol. 33, pp. 62-69, 2011.

[14] A. A. Mullick, N. K. Musampa, A. G. Feldman, and M. F. Levin, "Stretch reflex spatial threshold measure discriminates between spasticity and rigidity," Clinical Neurophysiology, vol. 124, pp. 740-751, 2013.

[15] C. Stinear, "Prediction of recovery of motor function after stroke," The Lancet Neurology, vol. 9, pp. 1228-1232, 2010. 
[16] T. Finni, P. Komi, and J. Lukkariniemi, "Achilles tendon loading during walking: application of a novel optic fiber technique," European journal of applied physiology and occupational physiology, vol. 77, pp. 289-291, 1998.

[17] J. T. Dennerlein, "Finger flexor tendon forces are a complex function of finger joint motions and fingertip forces," Journal of Hand Therapy, vol. 18, pp. 120-127, 2005.

[18] A. Erdemir, S. McLean, W. Herzog, and A. J. van den Bogert, "Modelbased estimation of muscle forces exerted during movements," Clinical Biomechanics, vol. 22, pp. 131-154, 2007.

[19] H. Abdullah, C. Tarry, R. Datta, G. Mittal, and M. Abderrahim, "Dynamic biomechanical model for assessing and monitoring robotassisted upper-limb therapy," Journal of rehabilitation research and development, vol. 44, p. 43, 2007.

[20] T. Barker, C. Kirtley, and J. Ratanapinunchai, "Calculation of multisegment rigid body joint dynamics using MATLAB," Proceedings of the Institution of Mechanical Engineers, Part H: Journal of Engineering in Medicine, vol. 211, pp. 483-487, 1997.

[21] F. C. Van der Helm, "A finite element musculoskeletal model of the shoulder mechanism," Journal of biomechanics, vol. 27, pp. 551-569, 1994.

[22] M. G. Pandy, "Computer modeling and simulation of human movement," Annual review of biomedical engineering, vol. 3, pp. 245-273, 2001.

[23] C. Meskers, H. Vermeulen, J. De Groot, F. Van der Helm, and P. Rozing, "3D shoulder position measurements using a six-degree-of-freedom electromagnetic tracking device," Clinical Biomechanics, vol. 13, pp. 280-292, 1998.

[24] T. B. Wyller, U. Sveen, K. M. Sødring, A. M. Pettersen, and E. BautzHolter, "Subjective well-being one year after stroke," Clinical rehabilitation, vol. 11, pp. 139-145, 1997.

[25] F. Coupar, A. Pollock, P. Rowe, C. Weir, and P. Langhorne, "Predictors of upper limb recovery after stroke: a systematic review and metaanalysis," Clinical rehabilitation, vol. 26, pp. 291-313, 2012.

[26] H. Nakayama, H. Jørgensen, H. Raaschou, and T. S. Olsen, "Recovery of upper extremity function in stroke patients: the Copenhagen Stroke Study," Archives of Physical Medicine and Rehabilitation, vol. 75, p. 394, 1994.

[27] G. Kwakkel, B. J. Kollen, J. van der Grond, and A. J. Prevo, "Probability of Regaining Dexterity in the Flaccid Upper Limb Impact of Severity of Paresis and Time Since Onset in Acute Stroke," Stroke, vol. 34, pp. 21812186, 2003.

[28] A. G. Fisher, Assessment of motor and process skills: Three Star Press Fort Collins, Colorado, 1997.

[29] C. Collin, D. Wade, S. Davies, and V. Horne, "The Barthel ADL Index: a reliability study," Disability \& Rehabilitation, vol. 10, pp. 61-63, 1988. 
[30] A. Fugl-Meyer, L. Jääskö, I. Leyman, S. Olsson, and S. Steglind, "The post-stroke hemiplegic patient. 1. a method for evaluation of physical performance," Scandinavian journal of rehabilitation medicine, vol. 7, p. 13, 1975.

[31] J. H. Carr, R. B. Shepherd, L. Nordholm, and D. Lynne, "Investigation of a new motor assessment scale for stroke patients," Physical Therapy, vol. 65, pp. 175-180, 1985.

[32] T. Platz, C. Pinkowski, F. van Wijck, I.-H. Kim, P. di Bella, and G. Johnson, "Reliability and validity of arm function assessment with standardized guidelines for the Fugl-Meyer Test, Action Research Arm Test and Box and Block Test: a multicentre study," Clinical Rehabilitation, vol. 19, pp. 404-411, 2005.

[33] V. Mathiowetz, G. Volland, N. Kashman, and K. Weber, "Adult norms for the Box and Block Test of manual dexterity," The American Journal of Occupational Therapy, vol. 39, pp. 386-391, 1985.

[34] R. W. Bohannon and M. B. Smith, "Interrater reliability of a modified Ashworth scale of muscle spasticity," Physical therapy, vol. 67, pp. 206207, 1987.

[35] K.-c. Lin, L. Chuang, C. Wu, Y. Hsieh, and W. Chang, "Responsiveness and validity of three dexterous function measures in stroke rehabilitation," J Rehabil Res Dev, vol. 47, pp. 563-571, 2010.

[36] N. Yozbatiran, L. Der-Yeghiaian, and S. C. Cramer, "A standardized approach to performing the action research arm test," Neurorehabilitation and Neural Repair, vol. 22, pp. 78-90, 2008.

[37] I. Hsueh and C.-L. Hsieh, "Responsiveness of two upper extremity function instruments for stroke inpatients receiving rehabilitation," Clinical rehabilitation, vol. 16, pp. 617-624, 2002.

[38] D. J. Gladstone, C. J. Danells, and S. E. Black, "The Fugl-Meyer assessment of motor recovery after stroke: a critical review of its measurement properties," Neurorehabilitation and Neural Repair, vol. 16, pp. 232-240, 2002.

[39] J. Sanford, J. Moreland, L. R. Swanson, P. W. Stratford, and C. Gowland, "Reliability of the Fugl-Meyer assessment for testing motor performance in patients following stroke," Physical therapy, vol. 73, pp. 447-454, 1993.

[40] M. A. Dettmann, M. T. Linder, and S. B. Sepic, "Relationships among walking performance, postural stability, and functional assessments of the hemiplegic patient," American Journal of Physical Medicine \& Rehabilitation, vol. 66, pp. 77-90, 1987.

[41] A. Pandyan, C. Price, H. Rodgers, M. Barnes, and G. Johnson, "Biomechanical examination of a commonly used measure of spasticity," Clinical Biomechanics, vol. 16, pp. 859-865, 2001.

[42] A. D. Pandyan, G. Johnson, C. Price, R. Curless, M. Barnes, and H. Rodgers, "A review of the properties and limitations of the Ashworth and modified Ashworth Scales as measures of spasticity," Clinical rehabilitation, vol. 13, pp. 373-383, 1999. 
[43] J. M. Gregson, M. J. Leathley, A. P. Moore, T. L. Smith, A. K. Sharma, and C. L. Watkins, "Reliability of measurements of muscle tone and muscle power in stroke patients," Age and ageing, vol. 29, pp. 223-228, 2000.

[44] N. N. Ansari, S. Naghdi, H. Moammeri, and S. Jalaie, "Ashworth scales are unreliable for the assessment of muscle spasticity," Physiotherapy theory and practice, vol. 22, pp. 119-125, 2006.

[45] D. Staudenmann, K. Roeleveld, D. F. Stegeman, and J. H. Van Dieën, "Methodological aspects of SEMG recordings for force estimation-a tutorial and review," Journal of Electromyography and Kinesiology, vol. 20, pp. 375-387, 2010.

[46] P. W. Duncan, S. M. Lai, and J. Keighley, "Defining post-stroke recovery: implications for design and interpretation of drug trials," Neuropharmacology, vol. 39, p. 835, 2000.

[47] P. Langhorne, F. Coupar, and A. Pollock, "Motor recovery after stroke: a systematic review," Lancet neurology, vol. 8, p. 741, 2009.

[48] G. KWAKKEL, R. C. WAGENAAR, B. J. KOLLEN, and G. J. LANKHORST, "Predicting disability in stroke-a critical review of the literature," Age and ageing, vol. 25, pp. 479-489, 1996.

[49] W. H. Organization. (2002). Towards a Common Language for Functioning, Disability and Health: International Classification on Functioning, Disability and Health. Available: http://www.who.int/classifications/icf/training/icfbeginnersguide.pdf

[50] R. W. BOHANNON, "ADEQUACY OF SIMPLE MEASURES FOR CHARACTERIZING IMPAIRMENT IN UPPER LIMB STRENGTH FOLLOWING STROKE 1," Perceptual and motor skills, vol. 99, pp. 813-817, 2004.

[51] W. Durfee and P. Iaizzo, "Rehabilitation and muscle testing," Encyclopedia of Medical Devices and Instrumentation, 2006.

[52] R. W. Bohannon, "Muscle strength and muscle training after stroke," Journal of Rehabilitation Medicine, vol. 39, pp. 14-20, 2007.

[53] R. Bonita and R. Beaglehole, "Recovery of motor function after stroke," Stroke, vol. 19, pp. 1497-1500, 1988.

[54] J. P. Mohr, Stroke: pathophysiology, diagnosis, and management: Churchill Livingstone, 2004.

[55] M. F. Levin, J. A. Kleim, and S. L. Wolf, "What do motor "recovery" and "compensation" mean in patients following stroke?," Neurorehabilitation and Neural Repair, vol. 23, pp. 313-319, 2009.

[56] Z. Dvir, Isokinetics: muscle testing, interpretation, and clinical applications: Churchill Livingstone Tel-Aviv, 2004.

[57] S. Cuthbert and G. Goodheart, "On the reliability and validity of manual muscle testing: a literature review," Chiropractic \& Manual Therapies, vol. 15, p. 4, 2007. 
[58] E. D. Adrian and D. W. Bronk, "The discharge of impulses in motor nerve fibres Part II. The frequency of discharge in reflex and voluntary contractions," The Journal of Physiology, vol. 67, pp. i3-151, 1929.

[59] R. Merletti, A. Holobar, and D. Farina, "Analysis of motor units with high-density surface electromyography," Journal of Electromyography and Kinesiology, vol. 18, pp. 879-890, 2008.

[60] R. Merletti, L. Lo Conte, E. Avignone, and P. Guglielminotti, "Modeling of surface myoelectric signals. I. Model implementation," Biomedical Engineering, IEEE Transactions on, vol. 46, pp. 810-820, 1999.

[61] R. Merletti, S. H. Roy, E. Kupa, S. Roatta, and A. Granata, "Modeling of surface myoelectric signals. II. Model-based signal interpretation," Biomedical Engineering, IEEE Transactions on, vol. 46, pp. 821-829, 1999.

[62] R. Merletti, A. Botter, A. Troiano, E. Merlo, and M. A. Minetto, "Technology and instrumentation for detection and conditioning of the surface electromyographic signal: state of the art," Clinical biomechanics (Bristol, Avon), vol. 24, pp. 122-134, 2009.

[63] D. Staudenmann, I. Kingma, A. Daffertshofer, D. Stegeman, and J. Van Dieën, "Heterogeneity of muscle activation in relation to force direction: A multi-channel surface electromyography study on the triceps surae muscle," Journal of Electromyography and Kinesiology, vol. 19, pp. 882895, 2009.

[64] S. L. Pullman, D. S. Goodin, A. I. Marquinez, S. Tabbal, and M. Rubin, "Clinical utility of surface EMG Report of the Therapeutics and Technology Assessment Subcommittee of the American Academy of Neurology," Neurology, vol. 55, pp. 171-177, 2000.

[65] A. Erdemir, S. McLean, W. Herzog, and A. J. van den Bogert, "Modelbased estimation of muscle forces exerted during movements," Clinical biomechanics (Bristol, Avon), vol. 22, pp. 131-154, 2007.

[66] H.-T. Lin, F.-C. Su, H.-W. Wu, and K.-N. An, "Muscle forces analysis in the shoulder mechanism during wheelchair propulsion," Proceedings of the Institution of Mechanical Engineers, Part H: Journal of Engineering in Medicine, vol. 218, pp. 213-221, 2004.

[67] D. A. Winter, Biomechanics and motor control of human movement: Wiley. com, 2009.

[68] V. M. Zatsiorsky, Kinetics of human motion: Human Kinetics 1, 2002.

[69] B. A. Garner and M. G. Pandy, "The obstacle-set method for representing muscle paths in musculoskeletal models," Computer methods in biomechanics and biomedical engineering, vol. 3, pp. 1-30, 2000.

[70] B. A. GARNER and M. G. PANDY, "Musculoskeletal model of the upper limb based on the visible human male dataset," Computer methods in biomechanics and biomedical engineering, vol. 4, pp. 93-126, 2001.

[71] H. Hatze, "A general myocybernetic control model of skeletal muscle," Biological Cybernetics, vol. 28, pp. 143-157, 1978. 
[72] G. C. Agarwal, B. M. Berman, P. Lohnberg, and L. Stark, "Studies in Postural Control Systems Part II: Tendon Jerk Input," Systems Science and Cybernetics, IEEE Transactions on, vol. 6, pp. 122-126, 1970.

[73] A. Hill, "The heat of shortening and the dynamic constants of muscle," Proceedings of the Royal Society of London. Series B, Biological Sciences, vol. 126, pp. 136-195, 1938.

[74] M. SILVA and J. A. AMBROSIO, "Solution of redundant muscle forces in human locomotion with multibody dynamics and optimization tools," Mechanics based design of structures and machines, vol. 31, pp. 381-411, 2003.

[75] C. Quental, J. Folgado, J. Ambrosio, and J. Monteiro, "A multibody biomechanical model of the upper limb including the shoulder girdle," Multibody System Dynamics, vol. 28, pp. 83-108, Aug 2012.

[76] D. Tsirakos, V. Baltzopoulos, and R. Bartlett, "Inverse optimization: functional and physiological considerations related to the force-sharing problem," Critical Reviews ${ }^{\mathrm{TM}}$ in Biomedical Engineering, vol. 25, 1997.

[77] S. G. McLean, A. Su, and A. J. van den Bogert, "Development and validation of a 3-D model to predict knee joint loading during dynamic movement," TRANSACTIONS-AMERICAN SOCIETY OF MECHANICAL ENGINEERS JOURNAL OF BIOMECHANICAL ENGINEERING, vol. 125, pp. 864-874, 2003.

[78] D. G. Thelen, F. C. Anderson, and S. L. Delp, "Generating dynamic simulations of movement using computed muscle control," Journal of Biomechanics, vol. 36, pp. 321-328, 2003.

[79] S. L. Delp, "Three-dimensional dynamic simulation of total knee replacement motion during a step-up task," ASME Journal of Biomechanical Engineering, vol. 123, pp. 599-606, 2001.

[80] T. K. Koo and A. F. Mak, "Feasibility of using EMG driven neuromusculoskeletal model for prediction of dynamic movement of the elbow," Journal of Electromyography and Kinesiology, vol. 15, pp. 1226, 2005.

[81] D. Amarantini and L. Martin, "A method to combine numerical optimization and EMG data for the estimation of joint moments under dynamic conditions," Journal of Biomechanics, vol. 37, pp. 1393-1404, 2004.

[82] C. J. van Andel, N. Wolterbeek, C. A. M. Doorenbosch, D. H. E. J. Veeger, and J. Harlaar, "Complete 3D kinematics of upper extremity functional tasks," Gait \& Posture, vol. 27, pp. 120-127, Jan 2008.

[83] H. J. Luinge, P. H. Veltink, and C. T. M. Baten, "Ambulatory measurement of arm orientation," Journal of Biomechanics, vol. 40, pp. 78-85, 2007.

[84] C. Anglin and U. P. Wyss, "Review of arm motion analyses," Proceedings of the Institution of Mechanical Engineers Part H-Journal of Engineering in Medicine, vol. 214, pp. 541-555, 2000. 
[85] J. Yang, X. Feng, J. H. Kim, and S. Rajulu, "Review of biomechanical models for human shoulder complex," International Journal of Human Factors Modelling and Simulation, vol. 1, pp. 271-293, 2010.

[86] A. Kontaxis, A. G. Cutti, G. R. Johnson, and H. E. J. Veeger, "A framework for the definition of standardized protocols for measuring upper-extremity kinematics," Clinical Biomechanics, vol. 24, pp. 246253, Mar 2009.

[87] K. Abdel-Malek, J. Yang, T. Marler, S. Beck, A. Mathai, X. Zhou, et al., "Towards a new generation of virtual humans," International Journal of Human Factors Modelling and Simulation, vol. 1, pp. 2-39, 2006.

[88] N. Kloopcar and J. Lenarcic, "Kinematic model for determination of human arm reachable workspace," Meccanica, vol. 40, pp. 203-219, Apr 2005.

[89] A. E. Engin and S. Tümer, "Three-dimensional kinematic modelling of the human shoulder complex--Part I: Physical model and determination of joint sinus cones," Journal of Biomechanical Engineering, vol. 111, p. 107, 1989.

[90] G. Wu, F. C. T. van der Helm, H. E. J. Veeger, M. Makhsous, P. Van Roy, C. Anglin, et al., "ISB recommendation on definitions of joint coordinate systems of various joints for the reporting of human joint motion - Part II: shoulder, elbow, wrist and hand," Journal of Biomechanics, vol. 38, pp. 981-992, May 2005.

[91] G. Pronk and F. Van der Helm, "The palpator: an instrument for measuring the positions of bones in three dimensions," Journal of medical engineering \& technology, vol. 15, pp. 15-20, 1991.

[92] G. Johnson and J. Anderson, "Measurement of three-dimensional shoulder movement by an electromagnetic sensor," Clinical Biomechanics, vol. 5, pp. 131-136, 1990.

[93] M. L. Pearl, S. L. Harris, S. B. Lippitt, J. A. Sidles, D. T. Harryman II, and F. A. Matsen III, "A system for describing positions of the humerus relative to the thorax and its use in the presentation of several functionally important arm positions," Journal of Shoulder and Elbow Surgery, vol. 1, pp. 113-118, 1992.

[94] C. Anglin and U. P. Wyss, "Arm motion and load analysis of sit-to-stand, stand-to-sit, cane walking and lifting," Clinical Biomechanics, vol. 15, pp. 441-448, 2000.

[95] D. P. Romilly, C. Anglin, R. G. Gosine, C. Hershler, and S. U. Raschke, "A functional task analysis and motion simulation for the development of a powered upper-limb orthosis," Rehabilitation Engineering, IEEE Transactions on, vol. 2, pp. 119-129, 1994.

[96] S. S. Rao, E. L. Bontrager, J. Gronley, C. J. Newsam, and J. Perry, "Threedimensional kinematics of wheelchair propulsion," Rehabilitation Engineering, IEEE Transactions on, vol. 4, pp. 152-160, 1996.

[97] T. Barker, A. Nicol, I. Kelly, and J. Paul, "Three-dimensional joint coordination strategies of the upper limb during functional activities," 
Proceedings of the Institution of Mechanical Engineers, Part H: Journal of Engineering in Medicine, vol. 210, pp. 17-26, 1996.

[98] A. G. Cutti, A. Giovanardi, L. Rocchi, A. Davalli, and R. Sacchetti, "Ambulatory measurement of shoulder and elbow kinematics through inertial and magnetic sensors," Medical \& Biological Engineering \& Computing, vol. 46, pp. 169-178, Feb 2008.

[99] C. F. Beaulieu, D. K. Hodge, A. G. Bergman, K. Butts, B. L. Daniel, C. L. Napper, et al., "Glenohumeral relationships during physiologic shoulder motion and stress testing: initial experience with open MR imaging and active imaging-plane registration1," Radiology, vol. 212, pp. 699-705, 1999.

[100] J. H. de Groot, "The variability of shoulder motions recorded by means of palpation," Clinical Biomechanics, vol. 12, pp. 461-472, 1997.

[101] P. I. Corke, "A robotics toolbox for MATLAB," Robotics \& Automation Magazine, IEEE, vol. 3, pp. 24-32, 1996.

[102] L. Chaitow, "The palpation reliability debate: the experts respond," Journal of Bodywork and Movement Therapies, vol. 6, p. 18, 2002.

[103] V. M. Zatsiorsky, "Kinematics of human motion. 1998," Human Kinetics, 2002.

[104] F. C. Van der Helm, "Analysis of the kinematic and dynamic behavior of the shoulder mechanism," Journal of biomechanics, vol. 27, pp. 527-550, 1994.

[105] M. D. K. Breteler, C. W. Spoor, and F. C. T. Van der Helm, "Measuring muscle and joint geometry parameters of a shoulder for modeling purposes," Journal of Biomechanics, vol. 32, pp. 1191-1197, Nov 1999.

[106] P. M. Ludewig, V. Phadke, J. P. Braman, D. R. Hassett, C. J. Cieminski, and R. F. LaPrade, "Motion of the Shoulder Complex During Multiplanar Humeral Elevation," Journal of Bone and Joint Surgery-American Volume, vol. 91A, pp. 378-389, Feb 2009.

[107] C. G. M. Meskers, H. M. Vermeulen, J. H. de Groot, F. C. T. van der Helm, and P. M. Rozing, "3D shoulder position measurements using a six-degree-of-freedom electromagnetic tracking device," Clinical Biomechanics, vol. 13, pp. 280-292, Jun-Jul 1998.

[108] A. R. Karduna, P. W. McClure, L. A. Michener, and B. Sennett, "Dynamic measurements of three-dimensional scapular kinematics: A validation study," Journal of Biomechanical Engineering-Transactions of the Asme, vol. 123, pp. 184-190, Apr 2001.

[109] J. H. de Groot and R. Brand, "A three-dimensional regression model of the shoulder rhythm," Clinical Biomechanics, vol. 16, pp. 735-743, Nov 2001.

[110] I. W. Charlton and G. R. Johnson, "A model for the prediction of the forces at the glenohumeral joint," Proceedings of the Institution of Mechanical Engineers Part H-Journal of Engineering in Medicine, vol. 220, pp. 801-812, Nov 2006. 
[111] M. Stokdijk, M. Biegstraaten, W. Ormel, Y. A. de Boer, H. E. J. Veeger, and P. M. Rozing, "Determining the optimal flexion-extension axis of the elbow in vivo - a study of interobserver and intraobserver reliability," Journal of Biomechanics, vol. 33, pp. 1139-1145, Sep 2000.

[112] M. Stokdijk, C. G. M. Meskers, H. E. J. Veeger, Y. A. de Boer, and P. M. Rozing, "Determination of the optimal elbow axis for evaluation of placement of prostheses," Clinical Biomechanics, vol. 14, pp. 177-184, Mar 1999.

[113] H. E. J. Veeger, B. Yu, K. N. An, and R. H. Rozendal, "Parameters for modeling the upper extremity," Journal of Biomechanics, vol. 30, pp. 647-652, Jun 1997.

[114] R. Shiba, C. Sorbie, D. W. Siu, J. T. Bryant, T. D. V. Cooke, and H. W. Wevers, "Geometry of the humeroulnar joint," Journal of orthopaedic research, vol. 6, pp. 897-906, 1988.

[115] E. Chao and B. Morrey, "Three-dimensional rotation of the elbow," Journal of Biomechanics, vol. 11, pp. 57-73, 1978.

[116] I. M. Chen, G. L. Yang, C. T. Tan, and S. H. Yeo, "Local POE model for robot kinematic calibration," Mechanism and Machine Theory, vol. 36, pp. 1215-1239, Nov-Dec 2001.

[117] Q. Yuan and I.-M. Chen, "Simultaneous Localization and Capture with velocity information," in Intelligent Robots and Systems (IROS), 2011 IEEE/RSJ International Conference on, 2011, pp. 2935-2940.

[118] Q. Yuan, I.-M. Chen, and S. P. Lee, "SLAC: 3D localization of human based on kinetic human movement capture," in Robotics and Automation (ICRA), 2011 IEEE International Conference on, 2011, pp. 848-853.

[119] J. M. McCarthy, Introduction to theoretical kinematics, 1990.

[120] H. E. J. Veeger and B. Yu, "Orientation of axes in the elbow and forearm for biomechanical modelling," Proceedings of the 1996 Fifteenth Southern Biomedical Engineering Conference, pp. 377-380, 1996.

[121] E. V. Biryukova, A. Roby-Brami, A. A. Frolov, and M. Mokhtari, "Kinematics of human arm reconstructed from spatial tracking system recordings," Journal of Biomechanics, vol. 33, pp. 985-995, Aug 2000.

[122] V. Zatsiorsky and V. Seluyanov, "The mass and inertia characteristics of the main segments of the human body," Biomechanics VIII-B, vol. 56, pp. 1152-1159, 1983.

[123] P. De Leva, "Adjustments to Zatsiorsky-Seluyanov's segment inertia parameters," Journal of Biomechanics, vol. 29, pp. 1223-1230, 1996.

[124] R. J. Schilling, Fundamentals of robotics: analysis and control: Simon \& Schuster Trade, 1996.

[125] C. Anglin and U. Wyss, "Review of arm motion analyses," Proceedings of the Institution of Mechanical Engineers, Part H: Journal of Engineering in Medicine, vol. 214, pp. 541-555, 2000.

[126] R. H. Jensen and D. T. Davy, "An investigation of muscle lines of action about the hip: A centroid line approach vs the straight line approach," Journal of Biomechanics, vol. 8, pp. 103-110, 1975. 
[127] A. Hill, "The heat of shortening and the dynamic constants of muscle," Proceedings of the Royal Society of London B: Biological Sciences, vol. 126, pp. 136-195, 1938.

[128] T. Siebert, C. Rode, W. Herzog, O. Till, and R. Blickhan, "Nonlinearities make a difference: comparison of two common Hill-type models with real muscle," Biological cybernetics, vol. 98, pp. 133-143, 2008.

[129] R. Alexander, M. B. Bennett, T. M. Casey, T. L. Daniel, M. E. DeMont, D. Grunbaum, et al., Mechanics of Animal Locomotion: Springer Berlin Heidelberg, 2011.

[130] M. G. Pandy, "4 Moment A rm of a Muscle Force," Exercise and sport sciences reviews, vol. 27, pp. 79-118, 1999.

[131] J. C. Otis, C.-C. Jiang, T. L. Wickiewicz, M. G. Peterson, R. F. Warren, and T. J. Santner, "Changes in the moment arms of the rotator cuff and deltoid muscles with abduction and rotation," Journal of Bone and Joint Surgery-A-American Volumes, vol. 76, pp. 667-676, 1994.

[132] J. Liu, R. Hughes, W. Smutz, G. Niebur, and K. Nan-An, "Roles of deltoid and rotator cuff muscles in shoulder elevation," Clinical Biomechanics, vol. 12, pp. 32-38, 1997.

[133] D. K. Kuechle, S. R. Newman, E. Itoi, B. F. Morrey, and K.-N. An, "Shoulder muscle moment arms during horizontal flexion and elevation," Journal of Shoulder and Elbow Surgery, vol. 6, pp. 429-439, 1997.

[134] W. M. Murray, S. L. Delp, and T. S. Buchanan, "Variation of muscle moment arms with elbow and forearm position," Journal of biomechanics, vol. 28, pp. 513-525, 1995.

[135] K. R. Holzbaur, W. M. Murray, and S. L. Delp, "A model of the upper extremity for simulating musculoskeletal surgery and analyzing neuromuscular control," Annals of biomedical engineering, vol. 33, pp. 829-840, 2005.

[136] P. Binding, A. Jinha, and W. Herzog, "Analytic analysis of the force sharing among synergistic muscles in one-and two-degree-of-freedom models," Journal of Biomechanics, vol. 33, pp. 1423-1432, 2000.

[137] T. A. Wren, K. P. Do, S. A. Rethlefsen, and B. Healy, "Cross-correlation as a method for comparing dynamic electromyography signals during gait," Journal of biomechanics, vol. 39, pp. 2714-2718, 2006.

[138] L. Li and G. E. Caldwell, "Coefficient of cross correlation and the time domain correspondence," Journal of Electromyography and Kinesiology, vol. 9, pp. 385-389, 1999.

[139] L.-Q. Zhang, G. Wang, T. Nishida, D. Xu, J. A. Sliwa, and W. Z. Rymer, "Hyperactive tendon reflexes in spastic multiple sclerosis: measures and mechanisms of action," Archives of physical medicine and rehabilitation, vol. 81, pp. 901-909, 2000.

[140] C. Sköld, R. Levi, and Å. Seiger, "Spasticity after traumatic spinal cord injury: nature, severity, and location," Archives of physical medicine and rehabilitation, vol. 80, pp. 1548-1557, 1999. 
[141] R. Wartenberg, "Pendulousness of the legs as a diagnostic test," Neurology, vol. 1, pp. 18-18, 1951.

[142] E. G. Fowler, A. I. Nwigwe, and T. W. Ho, "Sensitivity of the pendulum test for assessing spasticity in persons with cerebral palsy," Developmental Medicine \& Child Neurology, vol. 42, pp. 182-189, 2000.

[143] T. Bajd and L. Vodovnik, "Pendulum testing of spasticity," Journal of Biomedical Engineering, vol. 6, pp. 9-16, 1984.

[144] M. Jamshidi and A. W. Smith, "Clinical measurement of spasticity using the pendulum test: comparison of electrogoniometric and videotape analyses," Archives of physical medicine and rehabilitation, vol. 77, pp. 1129-1132, 1996.

[145] J. W. Fee and F. Miller, "The leg drop pendulum test performed under general anesthesia in spastic cerebral palsy," Developmental Medicine \& Child Neurology, vol. 46, pp. 273-281, 2004.

[146] I. White and H. Dulin, "Validity of the Pendulum Test to Measure Quadriceps Spasticity in Children Diagnosed with Cerebral Palsy," 2007.

[147] W. A. Lee, A. Boughton, and W. Z. Rymer, "Absence of stretch reflex gain enhancement in voluntarily activated spastic muscle," Experimental neurology, vol. 98, pp. 317-335, 1987.

[148] R. Powers, J. Marder-Meyer, and W. Rymer, "Quantitative relations between hypertonia and stretch reflex threshold in spastic hemiparesis," Annals of neurology, vol. 23, pp. 115-124, 1988.

[149] D. Kosarov, "The reaction time of single motor units in the human muscle," Agressologie: revue internationale de physio-biologie et de pharmacologie appliquees aux effets de l'agression, vol. 20, pp. 279-285, 1978.

[150] M. F. Levin, R. W. Selles, M. H. Verheul, and O. G. Meijer, "Deficits in the coordination of agonist and antagonist muscles in stroke patients: implications for normal motor control," Brain research, vol. 853, pp. 352369, 2000.

[151] T. G. Hornby, J. H. Kahn, M. Wu, and B. D. Schmit, "Temporal facilitation of spastic stretch reflexes following human spinal cord injury," The Journal of physiology, vol. 571, pp. 593-604, 2006.

[152] J. Colebatch, S. Gandevia, and P. Spira, "Voluntary muscle strength in hemiparesis: distribution of weakness at the elbow," Journal of Neurology, Neurosurgery \& Psychiatry, vol. 49, pp. 1019-1024, 1986.

[153] L. Dipietro, M. Ferraro, J. J. Palazzolo, H. I. Krebs, B. T. Volpe, and N. Hogan, "Customized interactive robotic treatment for stroke: EMGtriggered therapy," IEEE Transactions on Neural Systems and Rehabilitation Engineering, vol. 13, pp. 325-334, 2005.

[154] J. M. Bland and D. Altman, "Statistical methods for assessing agreement between two methods of clinical measurement," The lancet, vol. 327, pp. 307-310, 1986. 
[155] A. F. d. C. Hamilton, K. E. Jones, and D. M. Wolpert, "The scaling of motor noise with muscle strength and motor unit number in humans," Experimental Brain Research, vol. 157, pp. 417-430, 2004.

[156] A. D. Pandyan, C. I. Price, M. P. Barnes, and G. R. Johnson, "A biomechanical investigation into the validity of the modified Ashworth Scale as a measure of elbow spasticity," Clinical Rehabilitation, vol. 17, pp. 290-294, 2003. 